\title{
THE OUTCOME OF HIP EXERCISE IN PATELLOFEMORAL PAIN: A SYSTEMATIC REVIEW
}

\section{$\underline{\text { Abstract }}$}

Patellofemoral pain (PFP) is one of the most common lower extremity conditions seen in clinical practice. Current evidence shows that there are hip strength deficits, delayed onset and shorter activation of gluteus medius in people with PFP. The aim of this review was to systematically review the literature to investigate the outcome of hip exercise in people with PFP.

\section{Method}

AMED, CINAHL, Cochrane, EMBASE, PEDro, Pubmed, Science direct and SPORTDiscus databases were searched from inception to November 2014 for RCTs, non-randomised studies and case studies. Two independent reviewers assessed each paper for inclusion and quality.

\section{$\underline{\text { Results }}$}

Twenty one papers were identified;eighteen investigating strengthening exercise, two investigating the effect of neuromuscular exercise and one study investigated the effect of hip exercise for the prevention of PFP.

Hip and knee strengthening programmes were shown to be equally effective. Limited evidence indicates that the addition of hip exercise to an exercise programme is beneficial. Limited evidence demonstrates that motor skill retraining in a participant group who displayed abnormal hip alignment in running improves pain.

\section{Conclusion}

The evidence consistently demonstrated that both hip strengthening and neuromuscular exercise has a beneficial effect on pain and function in people with PFP. Strengthening exercise predominantly addressed abductor and external rotator muscle groups. 
A consensus from PFP researchers for standardisation of methodology is recommended to enable meaningful comparison between trials.

Keywords: patellofemoral, hip, neuromuscular, exercise, review

\section{INTRODUCTION}

Patellofemoral pain (PFP) is characterised by retropatellar or peripatellar pain associated with activities involving lower limb loading such as running, jumping, sustained sitting, kneeling, ascending or descending stairs, and squatting (Davis and Powers, 2010; Nijs et al., 2006). It is a common musculoskeletal disorder (Witvrouw etal., 2000) and was the most common overuse running injury in a prospective study of 2002 runners, accounting for $37.4 \%$ of knee injuries (Taunton et al., 2002). PFP has a reported incidence ranging from $3-40 \%$ of the population (Callaghan and Selfe, 2007) and females are 2.23 times more likely to develop the condition (Boling et al., 2010). It is not uncommon for patients to have long term symptoms. It was shown that $80 \%$ people with PFP who had completed a rehabilitation programme reported pain at a five year follow up, and $74 \%$ had reduced their physical activity level (Blond and Hansen, 1998). This may be due to underlying factors that contribute to the development of PFP not being addressed.

It is widely accepted that PFP is a multi-factorial condition (Powers,2012). There is increasing evidence that proximal factors may be associated with the pathogenesis of PFP.Biomechanical studies have shown that excessive femoral internal rotation in weight bearing leads to increased lateral patellar tracking, reduction in patellofemoral contact area (Besier et al., 2008; Huberti and Hayes, 1984; Lee et al., 2003; Li et al., 2004; Salsich and Perman, 2007) and increased lateral patellofemoral joint stress (Souza et al., 2010). This is proposed to lead to change in the patellofemoral joint articular cartilage, overloading the subchondral bone, causing pain (Powers, 2012). 
It has been proposed that that there are hip strength deficits in adults with PFP(Rathleff et al., 2014). However, evidence demonstrating that reduced muscle strength is accompanied by altered hip kinematics in PFP is conflicting, with some studies showing an association between a reduction in muscle strength with altered kinematics (Boling and Padua, 2013; Souza and Powers, 2009; Nakagawa et al., 2012) and others that there is not (Willson et al., 2008). There is delayed onset and shorter activation of gluteus medius in adults with PFP(Barton et al., 2013) and some evidence to show that altered gluteal muscle activation patterns accompanies altered hip kinematics in PFP (Souza and Powers 2009; Nakagawa et al., 2012). It follows that both strength and neuromuscular exercises of the gluteal muscles may be important factors to include in the management of PFP. A recent Cochrane review demonstrated consistent support for exercise in PFP (van der Heijden et al., 2015), but did not include neuromuscular exercise. This review included RCTs and quasirandomised studies. However, clinical decision making based on evidence based medicine should not be confined to RCTs; all available evidence should be considered and synthesised (Doherty, 2005; Koes and Hoving 1998). Observational studies with lower rigour but with higher generalisability may be of more clinical value (Milanese, 2011; Berbano and Baxi 2012) and when properly conducted with rigorous methods can be valuable in clinical research (Grossman and Mackenzie, 2005; Baker, 2011; Sharp, 1998). A more inclusive review of all proximal exercise will aid in the clinical management of PFP.

The aim of this review was to evaluate the effect of hip strengthening and neuromuscular exercise in people with PFP, providing clinicians with information to help plan effective management. 


\section{METHODOLOGY}

\section{Search strategy}

A systematic literature search was conducted of the electronic databases AMED, CINAHL, the Cochrane database, EMBASE, PEDro, Pubmed, Science direct and SPORTDiscus from their inception to November 2014. A search strategy from the Cochrane review on exercise therapy on PFP (Heintjes et al., 2003) was used for diagnosis terms and combined with key terms glute or proximal or hip or trunk; and exercise or rehabilitation and strength or endurance or motor control. A secondary search of relevant journals identified from related published research articles was also undertaken. These included Journal of Orthopaedic and Sports Physical Therapy, American Journal of Sports Medicine, British Journal of Sports Medicine and Journal of Sports Rehabilitation.

A search of the grey literature was undertaken using the databases WHO International Clinical Trials registry platform, OpenSIGLE, Zetoc and UK clinical research network study portfolio. Post-graduate theses were searched on the Index to theses database.

Relevant researchers in the field were contacted for information on unpublished research. The reference list of each article was hand searched to identify additional papers.

\section{Study eligibility}

Full text, English language articles were eligible.Randomised controlled trials (RCTs), non-randomised studies (NRS), cohort studies, case control studies and case studies investigating the effect of strengthening, endurance or neuromuscular exercise at the hip in subjects with patellofemoral pain were included, with at least one measure of pain, function or biomechanical outcome.

\section{$\underline{\text { Study Types }}$}

No restrictions were applied to the types of studies included. All available evidence was considered and synthesised to ensure a comprehensive review. 
A patellofemoral pain checklist was used (Table 1), with key inclusion and exclusion criteria for patellofemoral pain diagnosis (Barton et al.,2010). Studies investigating patellofemoral instability or patellofemoral osteoarthritis were excluded. There was no restriction on gender or age limits.

\section{$\underline{\text { Review process }}$}

Identified studies were downloaded into the bibliographic software programme Endnote Version X5 reference manager (Thomson Reuters). All identified titles and abstracts and subsequent full text articles were screened for eligibility. The final decision about inclusion was made by two independent researchers. A third researcher was consulted if a consensus was not reached. The researchers were not blinded to either source or author.

\section{Data extraction}

Data on the study design; participant characteristics; specific exercise; position; repetitions; frequency; intensity and outcome measures was extracted by two investigators.

\section{Methodological Quality assessment}

The PEDro scale (www.pedro.org.au) was used to assess the RCTs and NRS. The observational studies were assessed by appraisal tools from the Critical Appraisal Skills Programme (CASP) (www.casp-uk.net). The Oxford Centre of Evidence Medicine (CEBM) (www.cebm.net) appraisal tool for a case study was used for single case studies. Following the quality assessment a level of evidence was awarded for each of the studies, whichwas downgraded if there were serious limitations ("The Oxford 2011 Levels of Evidence").

\section{$\underline{\text { RESULTS }}$}

The initial search identified 1090 potentially relevant articles, of which 1062 were excluded based on title and abstract (figure 1). Full texts of28 articles were obtained; of these seven were excluded. 
One study provided detailed data from participants in a previous study; the data from these two papers were combined (Willy et al., 2012; Willy and Davis, 2013). This left 21 papers fulfilling the eligibility criteria. 


\section{Figure 1.}

\section{Flow diagram to illustrate the search results}

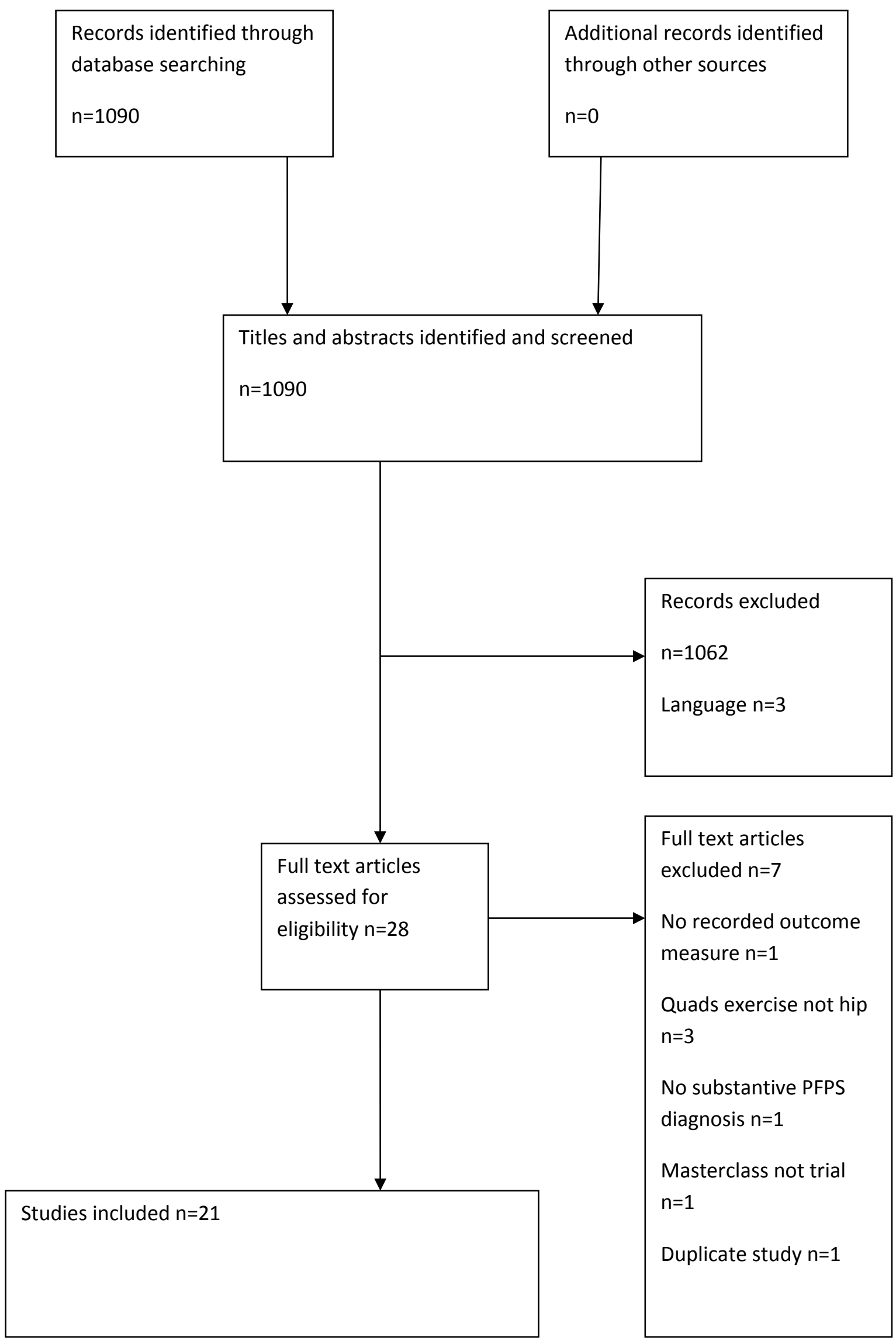




\section{Quality Assessment}

The methodological assessment of the reviewed papers is summarised in tables 2-4. The scores on the PEDro scale for RCTs ranged between three and nine out of a possible eleven. The main limitation was a lack of blinding of participants and therapists. One study did not meet the PEDro criteria for randomisation as participants were sequentially allocated (Khayambashi et al., 2012). A further study (Baldon et al., 2014) used block randomisation with groups of four, with no stratification.

The three case control studies scored seven and eight on the CASP Case control score out of a possible eleven. All subjects were recruited by convenience sampling. Two studies used an asymptomatic control group (Boling et al., 2006; Ferber et al., 2011). Confounding factors were poorly addressed. Case series studies scored between four and nine out of ten. The main limitation was that one researcher was responsible for data analysis in all studies. 
Table 1 Patellofemoral Pain Diagnosis Checklist

\begin{tabular}{|c|c|c|c|c|c|c|c|c|}
\hline & $\begin{array}{l}\frac{\text { Inclusion }}{\text { Clear definition }} \\
\text { of location }\end{array}$ & $\begin{array}{l}\text { Insidious onset } \\
\text { unrelated to } \\
\text { trauma }\end{array}$ & $\begin{array}{l}\text { Symptoms } \\
\text { consistent with } \\
\text { diagnosis }\end{array}$ & $\begin{array}{l}\text { Exclusion } \\
\text { Previous knee } \\
\text { surgery }\end{array}$ & $\begin{array}{l}\text { Internal } \\
\text { derangement }\end{array}$ & $\begin{array}{l}\text { Ligamentous } \\
\text { instability }\end{array}$ & $\begin{array}{l}\text { Other sources of } \\
\text { anterior knee } \\
\text { pain }\end{array}$ & $\begin{array}{l}\text { Total } \\
\text { score }\end{array}$ \\
\hline $\begin{array}{l}\text { Avraham et al., } \\
2007\end{array}$ & $\mathrm{Y}$ & $\mathrm{Y}$ & $\mathrm{Y}$ & $\mathrm{N}$ & $Y$ & $\mathrm{~N}$ & $\mathrm{Y}$ & 5 \\
\hline Baldon et al., 2014 & $Y$ & $\mathrm{Y}$ & $Y$ & $Y$ & $Y$ & $\mathrm{Y}$ & $Y$ & 7 \\
\hline Boling et al., 2006 & $\mathrm{Y}$ & $\mathrm{Y}$ & $\mathrm{Y}$ & $\mathrm{Y}$ & $\mathrm{N}$ & $\mathrm{N}$ & $Y$ & 5 \\
\hline $\begin{array}{l}\text { Coppack et al., } \\
2011\end{array}$ & $\mathrm{Y}$ & $Y$ & $Y$ & $Y$ & $Y$ & $Y$ & $Y$ & 7 \\
\hline Dolak et al., 2011 & $\mathrm{Y}$ & $\mathrm{Y}$ & $\mathrm{Y}$ & $\mathrm{Y}$ & $\mathrm{Y}$ & $\mathrm{Y}$ & $\mathrm{Y}$ & 7 \\
\hline Earl et al., 2011 & $Y$ & $Y$ & $Y$ & $\mathrm{Y}^{* *}$ & $Y$ & $Y$ & $Y$ & 7 \\
\hline Ferberet al., 2011 & $\mathrm{Y}$ & $\mathrm{Y}$ & $\mathrm{Y}$ & $Y$ & $\mathrm{~N}$ & $\mathrm{~N}$ & $\mathrm{Y}$ & 5 \\
\hline Ferber et al., 2014 & $Y$ & $Y$ & $Y$ & $\mathrm{Y}$ & $\mathrm{Y}$ & $Y$ & $Y$ & 7 \\
\hline Fukuda et al., 2010 & $\mathrm{Y}$ & $\mathrm{N}$ & $Y$ & $\mathrm{Y}$ & $Y$ & $\mathrm{Y}$ & $Y$ & 6 \\
\hline Fukuda et al., 2012 & $\mathrm{Y}$ & $\mathrm{N}$ & $\mathrm{Y}$ & $\mathrm{Y}$ & $\mathrm{Y}$ & $\mathrm{Y}$ & $\mathrm{Y}$ & 6 \\
\hline Ismail et al., 2013 & $\mathrm{Y}$ & $\mathrm{Y}$ & $\mathrm{Y}$ & $\mathrm{Y}$ & $Y$ & $\mathrm{Y}$ & $Y$ & 7 \\
\hline $\begin{array}{l}\text { Khayambashi et } \\
\text { al., } 2012\end{array}$ & $Y$ & $\mathrm{~N}$ & $Y$ & $Y$ & $Y$ & $Y$ & $Y$ & 6 \\
\hline $\begin{array}{l}\text { Khayambashi et al., } \\
2014\end{array}$ & $Y$ & $\mathrm{~N}$ & $Y$ & $Y$ & $Y$ & $Y$ & $Y$ & 6 \\
\hline Lowry et al., 2008 & $\mathrm{~N}$ & $\mathrm{~N}$ & $\mathrm{~N}$ & $\mathrm{Y}$ & $\mathrm{Y}$ & $\mathrm{Y}$ & $\mathrm{N}$ & 3 \\
\hline Mascal et al., 2003 & $\mathrm{Y}$ & $\mathrm{Y}$ & $\mathrm{Y}$ & $\mathrm{N}$ & $\mathrm{Y}$ & $Y$ & $Y$ & 6 \\
\hline $\begin{array}{l}\text { Nakagawa et al., } \\
2008\end{array}$ & $Y$ & $Y$ & $Y$ & $Y$ & $Y$ & $Y$ & $\mathrm{Y}$ & 7 \\
\hline $\begin{array}{l}\text { Noehren et al., } \\
2011\end{array}$ & $Y$ & $Y$ & $\mathrm{~N}$ & $\mathrm{~N}$ & $\mathrm{~N}$ & $\mathrm{~N}$ & $\mathrm{~N}$ & 2 \\
\hline Razeghi et al., 2010 & $\mathrm{Y}$ & $\mathrm{Y}$ & $\mathrm{Y}$ & $\mathrm{Y}^{*}$ & $Y$ & $\mathrm{Y}$ & $Y$ & 7 \\
\hline Song et al., 2009 & $\mathrm{Y}$ & $\mathrm{Y}$ & $\mathrm{Y}$ & $\mathrm{Y}$ & $\mathrm{N}$ & $\mathrm{N}$ & $\mathrm{Y}$ & 5 \\
\hline Tyler et al., 2006 & $Y$ & $Y$ & $\mathrm{Y}$ & $\mathrm{Y}^{* *}$ & $\mathrm{Y}$ & $\mathrm{Y}$ & $Y$ & 7 \\
\hline Willy et al., 2012 & $Y$ & $Y$ & $Y$ & $Y$ & $\mathrm{~N}$ & $\mathrm{~N}$ & $Y$ & 5 \\
\hline
\end{tabular}

*past 2 years** previous patellar surgery 
Table 2 Quality assessment RCTs PEDro scale

\begin{tabular}{|c|c|c|c|c|c|c|c|c|c|c|c|c|}
\hline & $\begin{array}{l}\text { Eligibility } \\
\text { specified }\end{array}$ & $\begin{array}{l}\text { Randomis- } \\
\text { ation }\end{array}$ & $\begin{array}{l}\text { Allocation } \\
\text { concealed }\end{array}$ & $\begin{array}{l}\text { Groups } \\
\text { similar at } \\
\text { baseline }\end{array}$ & $\begin{array}{l}\text { Subjects } \\
\text { blinded }\end{array}$ & $\begin{array}{l}\text { Therapists } \\
\text { blinded }\end{array}$ & $\begin{array}{l}\text { Assessors } \\
\text { blinded }\end{array}$ & $\begin{array}{l}\text { Key } \\
\text { outcome } \\
\text { obtained } \\
\text { from } 85 \%\end{array}$ & $\begin{array}{l}\text { Intention } \\
\text { to treat }\end{array}$ & $\begin{array}{l}\text { Between } \\
\text { group } \\
\text { statistical } \\
\text { outcome }\end{array}$ & $\begin{array}{l}\text { Point } \\
\text { measure }\end{array}$ & Total Score \\
\hline $\begin{array}{l}\text { Avraham et } \\
\text { al., } 2007\end{array}$ & $\mathrm{~N}$ & Y & $\mathrm{N}$ & $\mathrm{N}$ & $\mathrm{N}$ & $\mathrm{N}$ & $Y$ & $\mathrm{~N}$ & $\mathrm{~N}$ & $Y$ & $\mathrm{~N}$ & 3 \\
\hline $\begin{array}{l}\text { Baldon et al., } \\
2014\end{array}$ & $Y$ & $\mathrm{~N}$ & $Y$ & $Y$ & $Y$ & $\mathrm{~N}$ & $\mathrm{~N}$ & $Y$ & $Y$ & $Y$ & $Y$ & 8 \\
\hline $\begin{array}{l}\text { Coppack et } \\
\text { al., } 2011\end{array}$ & Y & Y & $Y$ & Y & $\mathrm{N}$ & $\mathrm{N}$ & $Y$ & $Y$ & $Y$ & Y & $Y$ & 9 \\
\hline $\begin{array}{l}\text { Dolak et al., } \\
2011\end{array}$ & Y & Y & $\mathrm{N}$ & Y & $\mathrm{N}$ & $\mathrm{N}$ & Y & $\mathrm{N}$ & Y & Y & $Y$ & 7 \\
\hline $\begin{array}{l}\text { Ferber et al., } \\
2014\end{array}$ & Y & Y & Y & Y & $\mathrm{N}$ & $\mathrm{N}$ & Y & $\mathrm{N}$ & Y & Y & $Y$ & 8 \\
\hline $\begin{array}{l}\text { Fukuda et al., } \\
2010\end{array}$ & Y & $Y$ & $\mathrm{Y}$ & Y & $\mathrm{N}$ & $\mathrm{N}$ & Y & $Y$ & $\mathrm{~N}$ & Y & $Y$ & 8 \\
\hline $\begin{array}{l}\text { Fukuda et al., } \\
2012\end{array}$ & $Y$ & $Y$ & $Y$ & $Y$ & $Y$ & $\mathrm{~N}$ & $\mathrm{~N}$ & $Y$ & $Y$ & Y & $Y$ & 9 \\
\hline $\begin{array}{l}\text { Ismail et al., } \\
2013\end{array}$ & $Y$ & $Y$ & $Y$ & $Y$ & $Y$ & $\mathrm{~N}$ & $\mathrm{~N}$ & $Y$ & $Y$ & $Y$ & $Y$ & 9 \\
\hline $\begin{array}{l}\text { Khayambashi } \\
\text { et al., } 2012\end{array}$ & $Y$ & $\mathrm{~N}$ & $\mathrm{~N}$ & $Y$ & $\mathrm{~N}$ & $\mathrm{~N}$ & $\mathrm{~N}$ & $Y$ & $Y$ & $Y$ & $Y$ & 6 \\
\hline $\begin{array}{l}\text { Khayambashi } \\
\text { et al., } 2014\end{array}$ & Y & $\mathrm{N}$ & $\mathrm{N}$ & Y & $\mathrm{N}$ & $\mathrm{N}$ & $\mathrm{N}$ & $Y$ & $Y$ & Y & $Y$ & 6 \\
\hline $\begin{array}{l}\text { Nakagawa et } \\
\text { al., } 2008\end{array}$ & $\mathrm{~N}$ & Y & $Y$ & Y & Y & $\mathrm{N}$ & $Y$ & $\mathrm{~N}$ & $Y$ & Y & $Y$ & 8 \\
\hline $\begin{array}{l}\text { Razeghi et al., } \\
2010\end{array}$ & Y & Y & $\mathrm{N}$ & $\mathrm{N}$ & $\mathrm{N}$ & $\mathrm{N}$ & $\mathrm{N}$ & $Y$ & $\mathrm{~N}$ & Y & $Y$ & 5 \\
\hline $\begin{array}{l}\text { Song et al., } \\
2009\end{array}$ & $Y$ & $Y$ & $Y$ & $Y$ & $\mathrm{~N}$ & $\mathrm{~N}$ & $Y$ & $Y$ & $Y$ & $Y$ & $Y$ & 9 \\
\hline
\end{tabular}

www.pedro.org.au 
Table 3 Quality assessment CASP Case control

\begin{tabular}{|c|c|c|c|c|c|c|c|c|c|c|c|c|}
\hline & $\begin{array}{l}\text { Clearly } \\
\text { focused } \\
\text { issue? }\end{array}$ & $\begin{array}{l}\text { Appropriate } \\
\text { method? }\end{array}$ & $\begin{array}{l}\text { Cohort } \\
\text { recruited in } \\
\text { an } \\
\text { acceptable } \\
\text { way? }\end{array}$ & $\begin{array}{l}\text { Exposure } \\
\text { accurately } \\
\text { measured? }\end{array}$ & $\begin{array}{l}\text { Outcome } \\
\text { accurately } \\
\text { measured? }\end{array}$ & $\begin{array}{l}\text { Confounding } \\
\text { issues } \\
\text { identified } \\
\text { and } \\
\text { accounted } \\
\text { for? }\end{array}$ & $\begin{array}{l}\text { Follow-up } \\
\text { complete } \\
\text { enough } \\
\text { and long } \\
\text { enough? }\end{array}$ & $\begin{array}{l}\text { Results- } \\
\text { bottom } \\
\text { line and } \\
\text { precise? }\end{array}$ & $\begin{array}{l}\text { Results } \\
\text { believable? }\end{array}$ & $\begin{array}{l}\text { Can the } \\
\text { results be } \\
\text { applied to } \\
\text { local } \\
\text { population? }\end{array}$ & $\begin{array}{l}\text { Do the } \\
\text { results fit } \\
\text { with other } \\
\text { available } \\
\text { evidence? }\end{array}$ & $\begin{array}{l}\text { Total } \\
\text { score }\end{array}$ \\
\hline $\begin{array}{l}\text { Boling et } \\
\text { al., } 2006\end{array}$ & $Y$ & $Y$ & $Y$ & $Y$ & $Y$ & $\mathrm{~N}$ & $Y$ & $\mathrm{~N}$ & $Y$ & $\mathrm{~N}$ & $Y$ & 8 \\
\hline $\begin{array}{l}\text { Ferber et } \\
\text { al., } 2011\end{array}$ & $Y$ & $Y$ & $Y$ & $Y$ & $Y$ & $\mathrm{~N}$ & $Y$ & $Y$ & $Y$ & $\mathrm{~N}$ & $Y$ & 9 \\
\hline
\end{tabular}

www.casp-uk.net 
Table 4 Critical Appraisal Exercise CEBM Case study quality

\begin{tabular}{|c|c|c|c|c|c|c|c|c|c|c|c|}
\hline & $\begin{array}{l}\text { Clearly } \\
\text { focused } \\
\text { question? }\end{array}$ & $\begin{array}{l}\text { Study design } \\
\text { appropriate? }\end{array}$ & $\begin{array}{l}\text { Setting and } \\
\text { subjects } \\
\text { representative? }\end{array}$ & $\begin{array}{l}\text { Researcher's } \\
\text { perspective } \\
\text { taken into } \\
\text { account? }\end{array}$ & $\begin{array}{l}\text { Methods for } \\
\text { collecting } \\
\text { data } \\
\text { described? }\end{array}$ & $\begin{array}{l}\text { Methods } \\
\text { analysing } \\
\text { data valid } \\
\text { and } \\
\text { reliable? }\end{array}$ & $\begin{array}{l}\text { Analysis } \\
\text { repeated by } \\
\text { more than one } \\
\text { researcher? }\end{array}$ & $\begin{array}{l}\text { Results } \\
\text { credible } \\
\text { and } \\
\text { relevant to } \\
\text { practice? }\end{array}$ & $\begin{array}{l}\text { Conclusions } \\
\text { drawn } \\
\text { justified by } \\
\text { results? }\end{array}$ & $\begin{array}{l}\text { Findings } \\
\text { transferable } \\
\text { to other } \\
\text { settings? }\end{array}$ & $\begin{array}{l}\text { Total } \\
\text { score }\end{array}$ \\
\hline $\begin{array}{l}\text { Earl et } \\
\text { al., } 2011\end{array}$ & $Y$ & $Y$ & $Y$ & $Y$ & $Y$ & $Y$ & $\mathrm{~N}$ & $Y$ & $Y$ & $Y$ & 9 \\
\hline $\begin{array}{l}\text { Lowry et } \\
\text { al., } 2008\end{array}$ & $\mathrm{~N}$ & $Y$ & $Y$ & $Y$ & $Y$ & $Y$ & $\mathrm{~N}$ & $\mathrm{~N}$ & $\mathrm{~N}$ & $\mathrm{~N}$ & 5 \\
\hline $\begin{array}{l}\text { Mascal et } \\
\text { al., } 2003\end{array}$ & $Y$ & Y & Y & Y & $Y$ & $\mathrm{~N}$ & $\mathrm{~N}$ & Y & Y & Y & 8 \\
\hline $\begin{array}{l}\text { Noehren } \\
\text { et al., } \\
2011 \\
\end{array}$ & $Y$ & $Y$ & $\mathrm{~N}$ & Y & $Y$ & $Y$ & $\mathrm{~N}$ & Y & $Y$ & $\mathrm{~N}$ & 7 \\
\hline $\begin{array}{l}\text { Tyler et } \\
\text { al., } 2006\end{array}$ & $Y$ & Y & Y & Y & $Y$ & $\mathrm{~N}$ & $\mathrm{~N}$ & $\mathrm{~N}$ & $Y$ & Y & 7 \\
\hline $\begin{array}{l}\text { Willy et } \\
\text { al., } 2012\end{array}$ & Y & $Y$ & $\mathrm{~N}$ & Y & $Y$ & Y & $\mathrm{N}$ & Y & $Y$ & Y & 8 \\
\hline $\begin{array}{l}\text { Willy et } \\
\text { al., } 2013\end{array}$ & $Y$ & $Y$ & $\mathrm{~N}$ & Y & $Y$ & Y & $\mathrm{N}$ & Y & Y & $Y$ & 8 \\
\hline
\end{tabular}

www.cebm.net 
Consistent support for the benefit of hip exercise in PFP was evident. Seven level two studies, two level three and eleven level four studies demonstrated a beneficial effect. Conversely, one level two paper demonstrated no benefit for hip exercise in PFP (see table 5).

Table 5 Levels of evidence.

\begin{tabular}{|l|l|l|l|}
\hline $\begin{array}{l}\text { CEBM level of } \\
\text { evidence }\end{array}$ & Level 2 & Level 3 & Level 4 \\
\hline $\begin{array}{l}\text { Beneficial effect } \\
\text { of hip exercise in } \\
\text { PFP }\end{array}$ & $\begin{array}{l}\text { Baldon et al., 2014 } \\
\text { Coppack et al., 2011 } \\
\text { Ferber et al., 2014 } \\
\text { Fukuda et al., 2010 } \\
\text { Fukuda et al., 2012 } \\
\text { Ismail et al.,2013 } \\
\text { Nakagawa et al., 2008 }\end{array}$ & $\begin{array}{l}\text { Dolak et al., 2011 } \\
\text { Khayambashi et al., } \\
2012\end{array}$ & $\begin{array}{l}\text { Avraham et al., 2007 } \\
\text { Boling et al., 2006 } \\
\text { Earl \& Hoch 2011 } \\
\text { Ferber et al., 2011 } \\
\text { Khayambashi et al., 2014 } \\
\text { Lowry et al., 2008 } \\
\text { Mascal et al.,2003 } \\
\text { Noehren et al .,2011 } \\
\text { Razheghi et al., 2010 } \\
\text { Tyler et al.,2006 } \\
\text { Willy et al., 2012 }\end{array}$ \\
\hline $\begin{array}{l}\text { No benefit of hip } \\
\text { exercise in PFP }\end{array}$ & Song et al., 2009 & & \\
\hline
\end{tabular}

www.cebm.net/ocebm-levels-of-evidence 


\section{Strengthening exercise}

Eighteen studies investigated strengthening exercise (table 6) including one prospective study investigating the prevention of anterior knee pain (Coppack et al., 2011).

753 PFP participants (578 females, 133 males, 42 genders unknown) with an age range between 14 and 50 years were included. Eight studies included females only; one study did not state gender. The number of participants in the exercise groups were generally small, ranging from 15-35 although there were two large RCTs with 111 and 759 proximal exercise participants. Studies were commonly underpowered; only six had sample size calculations that were adhered to.

Nine studies included participants with both bilateral and unilateral symptoms, five studies only included participants with unilateral symptoms, one study included participants with bilateral symptoms and two studies did not include this data. Five studies evaluated the most symptomatic knee, two studies the most painful knee on initial testing, and three studies evaluated both knees. Six studies investigated participants who did not regularly participate in exercise, four investigated participants who participated in sports; there was no available data on activity levels from the remaining studies. 
Table 6 Study characteristics: Strengthening exercise

\begin{tabular}{|c|c|c|c|c|c|c|c|c|}
\hline Study & Study design & Population & Sample size & $\begin{array}{l}\text { Sample size } \\
\text { calculation }\end{array}$ & Age & Gender & $\begin{array}{l}\text { Additional } \\
\text { inclusion criteria } \\
\text { PFP }\end{array}$ & Unilateral/bilateral PFP \\
\hline $\begin{array}{l}\text { Avraham et } \\
\text { al } 2007\end{array}$ & $\begin{array}{l}\text { RCT } \\
\text { Comparison of } \\
\text { three groups: Hip } \\
\text { orientated, knee } \\
\text { orientated and hip } \\
\text { and knee } \\
\text { orientated exercise }\end{array}$ & $\begin{array}{l}\text { No data on activity } \\
\text { levels }\end{array}$ & 42 & No & $\begin{array}{l}\text { Mean age } 35 \\
\text { years } \\
\text { No other } \\
\text { data } \\
\text { recorded }\end{array}$ & Unknown & Not specified & Unknown \\
\hline $\begin{array}{l}\text { Baldon et al } \\
2014\end{array}$ & $\begin{array}{l}\text { RCT } \\
\text { Comparison of two } \\
\text { groups: Functional } \\
\text { stabilisation } \\
\text { training (FST) = Hip, } \\
\text { lower limb and } \\
\text { trunk exercise and } \\
\text { Standard Training } \\
\text { (ST) = quadriceps } \\
\text { exercise }\end{array}$ & $\begin{array}{l}\text { Recreational } \\
\text { athletes }\end{array}$ & $\begin{array}{l}31 \\
16 \text { hip } \\
\text { group } \\
15 \\
\text { quadriceps } \\
\text { group }\end{array}$ & $\begin{array}{l}\text { Yes; } \\
\text { numbers at } 3 \\
\text { month follow- } \\
\text { up did not } \\
\text { equate to } \\
\text { sample size }\end{array}$ & $18-30$ years & female & $\begin{array}{l}\text { Duration }>8 \text { weeks } \\
\text { VAS }>3 / 10\end{array}$ & Unknown \\
\hline $\begin{array}{l}\text { Coppack et } \\
\text { al } 2011\end{array}$ & $\begin{array}{l}\text { RCT } \\
\text { Prospective study } \\
\text { for the prevention } \\
\text { of anterior knee } \\
\text { pain. } \\
\text { Intervention group } \\
\text { of gluteal and } \\
\text { quadriceps } \\
\text { exercise and } \\
\text { control group of } \\
\text { running and upper } \\
\text { body exercise. }\end{array}$ & Military recruits & $\begin{array}{l}1502 \\
759 \\
\text { intervention } \\
\text { group } \\
743 \text { control } \\
\text { group }\end{array}$ & $\begin{array}{l}\text { Numbers in } \\
\text { study did not } \\
\text { equate to } \\
\text { numbers } \\
\text { needed from } \\
\text { sample size } \\
\text { calculation }\end{array}$ & $17-25$ years & $\begin{array}{l}\text { male } \\
(1092) \\
\text { female } \\
(410)\end{array}$ & $\mathrm{N} / \mathrm{A}$ & $\mathrm{N} / \mathrm{A}$ \\
\hline $\begin{array}{l}\text { Dolak et al } \\
2011\end{array}$ & RCT & $\begin{array}{l}\text { No data on activity } \\
\text { levels. }\end{array}$ & 33 & No & $16-35$ years & female & Duration $>1$ month & Unilateral (17) \\
\hline
\end{tabular}




\begin{tabular}{|c|c|c|c|c|c|c|c|c|}
\hline & $\begin{array}{l}\text { Comparison of two } \\
\text { groups: initial hip } \\
\text { exercise group or } \\
\text { initial knee } \\
\text { exercise group } \\
\text { prior to same } \\
\text { functional } \\
\text { exercises }\end{array}$ & & $\begin{array}{l}17 \text { hip } \\
\text { group } \\
16 \text { knee } \\
\text { group }\end{array}$ & & & & & $\begin{array}{l}\text { bilateral (16) - most } \\
\text { painful limb during } \\
\text { initial testing used for } \\
\text { analysis }\end{array}$ \\
\hline $\begin{array}{l}\text { Ferber et al., } \\
2014\end{array}$ & $\begin{array}{l}\text { RCT } \\
\text { Comparison of two } \\
\text { groups: Hip \& core } \\
\text { exercise group or } \\
\text { knee exercise } \\
\text { group }\end{array}$ & $\begin{array}{l}\text { Recreationally } \\
\text { active }\end{array}$ & $\begin{array}{l}199 \\
111 \text { hip } \\
\text { group } \\
88 \text { knee } \\
\text { group }\end{array}$ & Yes & $29+/-7$ years & $\begin{array}{l}\text { male (66) } \\
\text { female } \\
(133)\end{array}$ & $\begin{array}{l}\text { Duration }>4 \text { weeks } \\
\text { VAS }>3 / 10\end{array}$ & $\begin{array}{l}\text { Unilateral / bilateral } \\
\text { No data re numbers } \\
\text { Bilateral- both limbs } \\
\text { exercised, most } \\
\text { affected limb included } \\
\text { in data }\end{array}$ \\
\hline $\begin{array}{l}\text { Fukuda et al } \\
2010\end{array}$ & $\begin{array}{l}\text { RCT } \\
\text { Comparison of } \\
\text { three groups: } \\
\text { Hip \& knee } \\
\text { exercise group, } \\
\text { knee exercise } \\
\text { group } \\
\text { Control group- no } \\
\text { exercise }\end{array}$ & Sedentary & $\begin{array}{l}70 \\
22 \text { hip } \\
\text { group } \\
23 \text { knee } \\
\text { group } \\
25 \text { control } \\
\text { group }\end{array}$ & Yes & $20-40$ years & female & $\begin{array}{l}\text { Duration }>3 \\
\text { months }\end{array}$ & Unilateral \\
\hline $\begin{array}{l}\text { Fukuda et al } \\
2012\end{array}$ & $\begin{array}{l}\text { RCT } \\
\text { Comparison of two } \\
\text { groups: knee } \\
\text { exercise group and } \\
\text { knee and hip } \\
\text { exercise group }\end{array}$ & Sedentary & $\begin{array}{l}54 \\
\text { knee group } \\
26 \\
\text { hip group } \\
28\end{array}$ & Yes & $20-40$ years & female & $\begin{array}{l}\text { Duration }>3 \\
\text { months }\end{array}$ & Unilateral \\
\hline $\begin{array}{l}\text { Ismail et al } \\
2013\end{array}$ & RCT & $\begin{array}{l}\text { No data on activity } \\
\text { levels }\end{array}$ & 32 & No & $18-30$ years & $\begin{array}{l}\text { male(9) } \\
\text { female(23) }\end{array}$ & Duration $>6$ weeks & Unilateral \\
\hline
\end{tabular}




\begin{tabular}{|c|c|c|c|c|c|c|c|c|}
\hline & $\begin{array}{l}\text { Comparison of two } \\
\text { groups: CKC } \\
\text { exercise and same } \\
\text { CKC exercise with } \\
\text { addition of OKC hip } \\
\text { exercise }\end{array}$ & & $\begin{array}{l}16 \text { per } \\
\text { group }\end{array}$ & & & & & \\
\hline $\begin{array}{l}\text { Khayambashi } \\
\text { et al } 2012\end{array}$ & $\begin{array}{l}\text { RCT } \\
\text { Comparison of two } \\
\text { groups: a hip } \\
\text { exercise group to a } \\
\text { no-exercise control } \\
\text { group who took } \\
\text { 1000mg Omega-3 } \\
\text { and } 400 \mathrm{mg} \text { calcium } \\
\text { daily }\end{array}$ & $\begin{array}{l}\text { Sedentary. } \\
\text { No previous } \\
\text { physiotherapy. }\end{array}$ & $\begin{array}{l}28 \\
14 \text { per } \\
\text { group }\end{array}$ & No & $\begin{array}{l}\text { Hip group } \\
28.9+/-5.8 \\
\text { years } \\
\text { Control } \\
\text { group } \\
30.5+/-4.8 \\
\text { years }\end{array}$ & female & $\begin{array}{l}\text { Duration }>6 \\
\text { months }\end{array}$ & $\begin{array}{l}\text { Bilateral- both knees } \\
\text { exercised \& tested }\end{array}$ \\
\hline $\begin{array}{l}\text { Nakagawa et } \\
\text { al } 2008\end{array}$ & $\begin{array}{l}\text { RCT } \\
\text { Comparison of two } \\
\text { groups: a } \\
\text { quadriceps } \\
\text { strengthening } \\
\text { group, a group } \\
\text { with the addition } \\
\text { of hip exercise }\end{array}$ & $\begin{array}{l}\text { No data re activity } \\
\text { levels. }\end{array}$ & 74 per group & No & $17-40$ years & $\begin{array}{l}\text { male(4) } \\
\text { female(10) }\end{array}$ & Duration $>4$ weeks & $\begin{array}{l}\text { Unilateral/bilateral. No } \\
\text { data re numbers. } \\
\text { Bilateral - most affected } \\
\text { limb included. }\end{array}$ \\
\hline $\begin{array}{l}\text { Razeghi et al } \\
2010\end{array}$ & $\begin{array}{l}\text { RCT } \\
\text { Comparison of two } \\
\text { groups: hip and } \\
\text { knee exercise } \\
\text { group and knee } \\
\text { exercise group }\end{array}$ & No data & $\begin{array}{l}33 \\
17 \text { hip and } \\
\text { knee } \\
16 \text { knee }\end{array}$ & No & $18-30$ years & female & Not specified & $\begin{array}{l}\text { Unilateral/bilateral } \\
\text { (52 knees) }\end{array}$ \\
\hline $\begin{array}{l}\text { Song et al } \\
2009\end{array}$ & $\begin{array}{l}\text { RCT } \\
\text { Comparison of } \\
\text { three groups: leg } \\
\text { press exercise } \\
\text { group, leg press } \\
\text { with addition of hip }\end{array}$ & No regular sports. & $\begin{array}{l}89 \\
\text { leg press } 30 \\
\text { leg press } \\
\text { with hip }\end{array}$ & Yes & $<50$ years & $\begin{array}{l}\text { male(20) } \\
\text { female(69) }\end{array}$ & $\begin{array}{l}\text { Duration }>1 \text { month } \\
\text { Excluded VAS } \\
>8 / 10\end{array}$ & $\begin{array}{l}\text { Unilateral/bilateral PFP. } \\
\text { No data re numbers. } \\
\text { Bilateral- most } \\
\text { symptomatic knee } \\
\text { included. Stratified } \\
\text { allocation re }\end{array}$ \\
\hline
\end{tabular}




\begin{tabular}{|c|c|c|c|c|c|c|c|c|}
\hline & $\begin{array}{l}\text { adduction and no- } \\
\text { exercise control } \\
\text { group }\end{array}$ & & $\begin{array}{l}\text { adduction } \\
29 \\
\text { control } \\
\text { group } 30\end{array}$ & & & & & $\begin{array}{l}\text { unilateral/bilateral } \\
\text { symptoms }\end{array}$ \\
\hline $\begin{array}{l}\text { Boling et al } \\
2006\end{array}$ & $\begin{array}{l}\text { Case control } \\
\text { Rehabilitation } \\
\text { programme of } \\
\text { weight bearing } \\
\text { exercise. } \\
\text { Matched no- } \\
\text { exercise control } \\
\text { group of } \\
\text { asymptomatics }\end{array}$ & $\begin{array}{l}\text { No data re activity } \\
\text { levels }\end{array}$ & $\begin{array}{l}28 \\
14 \text { exercise } \\
\text { group } \\
14 \text { no } \\
\text { exercise } \\
\text { group }\end{array}$ & No & $18-42$ years & $\begin{array}{l}\text { male (5) } \\
\text { female(9) }\end{array}$ & $\begin{array}{l}\text { Excluded if } \\
\text { duration }<2 \\
\text { months }\end{array}$ & $\begin{array}{l}\text { Unilateral/bilateral No } \\
\text { data re numbers } \\
\text { Bilateral- most affected } \\
\text { limb analysed. } \\
\text { Controls- R limb } \\
\text { analysed. }\end{array}$ \\
\hline $\begin{array}{l}\text { Earl and } \\
\text { Hoch } 2011\end{array}$ & $\begin{array}{l}\text { Case series } \\
\text { Rehabilitation } \\
\text { "proximal stability } \\
\text { programme" }\end{array}$ & $\begin{array}{l}\text { Participated in } \\
\text { exercise/sports }\end{array}$ & 28 & Yes & $\begin{array}{l}22.68+/-7.19 \\
\text { years }\end{array}$ & female & Not specified & $\begin{array}{l}\text { Unilateral/bilateral PFP. } \\
\text { No data re numbers. } \\
\text { Bilateral- most painful } \\
\text { limb tested }\end{array}$ \\
\hline $\begin{array}{l}\text { Ferber et al } \\
2011\end{array}$ & $\begin{array}{l}\text { Case control } \\
\text { Hip abductor } \\
\text { strengthening } \\
\text { group compared to } \\
\text { a no-exercise } \\
\text { control group of } \\
\text { asymptomatics }\end{array}$ & $\begin{array}{l}\text { Recreational } \\
\text { runners }\end{array}$ & $\begin{array}{l}25 \\
15 \text { PFP } \\
10 \text { control } \\
\text { group }\end{array}$ & Yes & $\begin{array}{l}\text { PFP } 35.2+/- \\
12.2 \text { years } \\
\text { Controls } \\
29.9+/-8.3 \\
\text { years }\end{array}$ & $\begin{array}{l}\text { male (5) } \\
\text { female(10) }\end{array}$ & $\begin{array}{l}\text { Excluded duration } \\
>2 \text { months } \\
\text { VAS }>3 / 10\end{array}$ & Unilateral \\
\hline $\begin{array}{l}\text { Khayambashi } \\
\text { et al } 2014\end{array}$ & $\begin{array}{l}\text { Case control study. } \\
\text { Comparison of hip } \\
\text { exercise group to a } \\
\text { quadriceps } \\
\text { exercise group }\end{array}$ & Sedentary & $\begin{array}{l}36 \\
18 \text { per } \\
\text { group }\end{array}$ & No & $\begin{array}{l}\text { Hip group } \\
28.2+/-7.9 \\
\text { years } \\
\text { Knee group } \\
27.3+/-6.7 \\
\text { years }\end{array}$ & $\begin{array}{l}\text { male(18) } \\
\text { female(18) }\end{array}$ & $\begin{array}{l}\text { Excluded duration } \\
<6 \text { months }\end{array}$ & $\begin{array}{l}\text { Unilateral(14) or } \\
\text { bilateral (22) } \\
\text { Limb reported to be } \\
\text { most painful during } \\
\text { initial testing evaluated }\end{array}$ \\
\hline
\end{tabular}




\begin{tabular}{|c|c|c|c|c|c|c|c|c|}
\hline & & & & & & & & \\
\hline $\begin{array}{l}\text { Lowry et al., } \\
2008\end{array}$ & $\begin{array}{l}\text { Case study } \times 5 \\
\text { Multi-modal } \\
\text { treatment } \\
\text { (combination of } \\
\text { thrust and non- } \\
\text { thrust } \\
\text { manipulation } \\
\text { directed at the } \\
\text { joints of the lower } \\
\text { quarter, trunk and } \\
\text { hip stabilization } \\
\text { exercises, patellar } \\
\text { taping, and foot } \\
\text { orthotics) }\end{array}$ & $\begin{array}{l}\text { No data re activity } \\
\text { levels }\end{array}$ & 5 & N/A & $\begin{array}{l}14,15,15,25, \\
50 \text { years }\end{array}$ & $\begin{array}{l}\text { male (3) } \\
\text { female (2) }\end{array}$ & Not specified & $\begin{array}{l}\text { Bilateral (3) } \\
\text { Unilateral (2) }\end{array}$ \\
\hline Mascal 2003 & $\begin{array}{l}\text { Case study } \times 2 \\
\text { subjects } \\
\text { (excluded one as } \\
\text { patellar } \\
\text { instability,previous } \\
\text { dislocation/positive } \\
\text { apprehension test) }\end{array}$ & Sedentary & 1 & N/A & 37 years & female & & Unilateral \\
\hline Tyler 2006 & $\begin{array}{l}\text { Case series } \\
\text { Hip flexibility and } \\
\text { strengthening } \\
\text { programme } \\
\end{array}$ & $\begin{array}{l}\text { No data re activity } \\
\text { levels. }\end{array}$ & 35 & No & $\begin{array}{l}33+/-16 \\
\text { years }\end{array}$ & $\begin{array}{l}\text { female(29) } \\
\text { male(6) }\end{array}$ & Duration $>4$ weeks & $\begin{array}{l}\text { Unilateral / bilateral } \\
\text { PFP. Bilateral ( } 8 \text { ) both } \\
\text { knees included- } 43 \\
\text { knees in study. }\end{array}$ \\
\hline
\end{tabular}

\section{PFP- Patellofemoral pain}

\section{RCT-randomised controlled trial}

\section{CKC- closed kinetic chain}

\section{OKC- open kinetic chain}


VAS - Visual analogue scale 


\section{Strengthening Exercise Protocols}

Widespread variation in the exercise protocols was evident (see table 7). Thirteen out of the eighteen studies compared exercise groups. Four investigated the addition of hip exercises to an exercise regime; three were additional open kinetic chain (OKC) hip exercises. Three studies compared hip and knee exercises.

Most hip protocols targeted the hip abductors and external rotators. In contrast, one study investigated isometric adduction (Song et al., 2009).Ten studies included both OKC and closed chain (CKC) exercises. Four used OKC only. Twelve studies included OKC abduction, and seven OKC external rotation. Thirteen hip protocols included CKC exercises; seven unilateral CKC exercise. All control group exercises included CKC exercise except one (Coppack et al., 2011), with three including unilateral CKC exercise and four, squats.

All studies progressed exercise except one (Razeghi et al., 2010). The criteria for progression in eleven studies was time, with six studies taking ability into account and two pain. Progression was achieved with increasing repetitions of exercise in five studies, increasing load in thirteen studies and increasing demand of the exercise in five studies.

Exercise was carried out three times a week in ten of the studies, with four prescribing more frequent and three less frequent exercise. One did not state frequency of exercise. Exercise programmes were carried out from three weeks to fourteen weeks; thirteen were at least six weeks duration. The majority of studies analysed the results at the end of the programme. Two studies evaluated the long term effects at six months and one additionally at one year (Fukuda et al., 2012). 
Table 7 Strengthening Exercise Protocols

\begin{tabular}{|c|c|c|c|c|c|c|}
\hline Study & Exercise & Progression & Additional exercise & $\begin{array}{l}\text { Time frame } \\
\text { for exercise }\end{array}$ & $\begin{array}{l}\text { Reps, intensity, } \\
\text { frequency }\end{array}$ & f/u period \\
\hline Avraham et al 2007 & $\begin{array}{l}\text { Knee group } \\
\text { 1. SLR } 10 \text { secs Hold } 10 \text { secs rest. } 7.5 \text { minutes } \\
\text { 2. Single leg squats } 7.5 \text { minutes } \\
\text { 3. TENS } 15 \text { minutes } \\
\text { Hip group } \\
\text { 1. Therapist assisted ITB stretch } 10 \text { secs } \\
\text { hold, } 10 \text { secs rest, } 3 \text { minutes } \\
\text { 2. Therapist assisted Hamstring stretch (SLR) } \\
\text { 10 secs hold, } 10 \text { secs rest, } 3 \text { minutes } \\
\text { 3. Hip external rotation. Ipsilateral side- } \\
\text { Iying, knee \& hip flexion } 90 \text { degrees, limb } \\
\text { over edge of bed. From full internal } \\
\text { rotation to } 30 \text { degrees external rotation. } \\
9 \text { minutes. } \\
\text { 4. TENS } 15 \text { minutes } \\
\text { Hip and knee group } \\
\text { 1. SLR } 10 \text { secs Hold } 10 \text { secs rest. } 3 \\
\text { minutes } \\
\text { 2. Single leg squats } 3 \text { minutes } \\
\text { 3. Therapist assisted ITB stretch } 10 \text { secs } \\
\text { hold, } 10 \text { secs rest, } 3 \text { minutes } \\
\text { 4. Therapist assisted Hamstring stretch } \\
\text { (SLR) } 10 \text { secs hold, } 10 \text { secs rest, } 3 \\
\text { minutes } \\
\text { 5. Hip external rotation. Ipsilateral side- } \\
\text { lying, knee } \& \text { hip flexion } 90 \text { degrees, } \\
\text { limb over edge of bed. From full } \\
\text { internal rotation to } 30 \text { degrees } \\
\text { external rotation. } 3 \text { minutes. }\end{array}$ & $\begin{array}{l}\text { Patients } \\
\text { instructed to } \\
\text { increase load, } \\
\text { time and reps as } \\
\text { able }\end{array}$ & $\begin{array}{l}\text { See individual group } \\
\text { data }\end{array}$ & 3 weeks & $\begin{array}{l}2 \mathrm{x} \text { week in } \\
\text { clinic, exercises } \\
\text { only at home } 4 \\
\mathrm{x} \text { week }\end{array}$ & 3 weeks \\
\hline
\end{tabular}




\begin{tabular}{|c|c|c|c|c|c|}
\hline & 6. TENS 15 minutes & & & & \\
\hline Baldon et al 2014 & 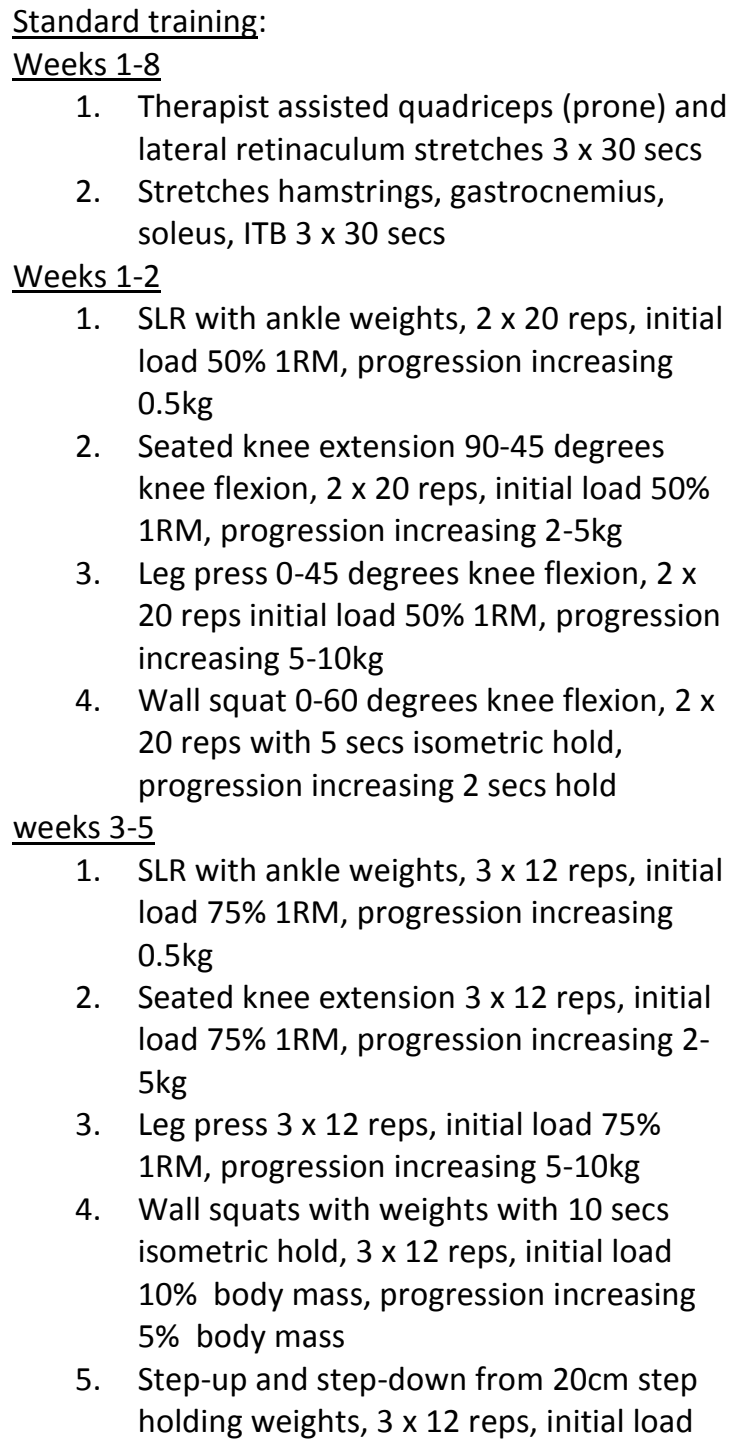 & $\begin{array}{l}\text { Time. } \\
\text { See individual } \\
\text { exercises for } \\
\text { reps, load } \\
\text { progression }\end{array}$ & 8 weeks & $3 \times$ week & $\begin{array}{l}8 \text { weeks } \\
\text { and } 3 \\
\text { months }\end{array}$ \\
\hline
\end{tabular}




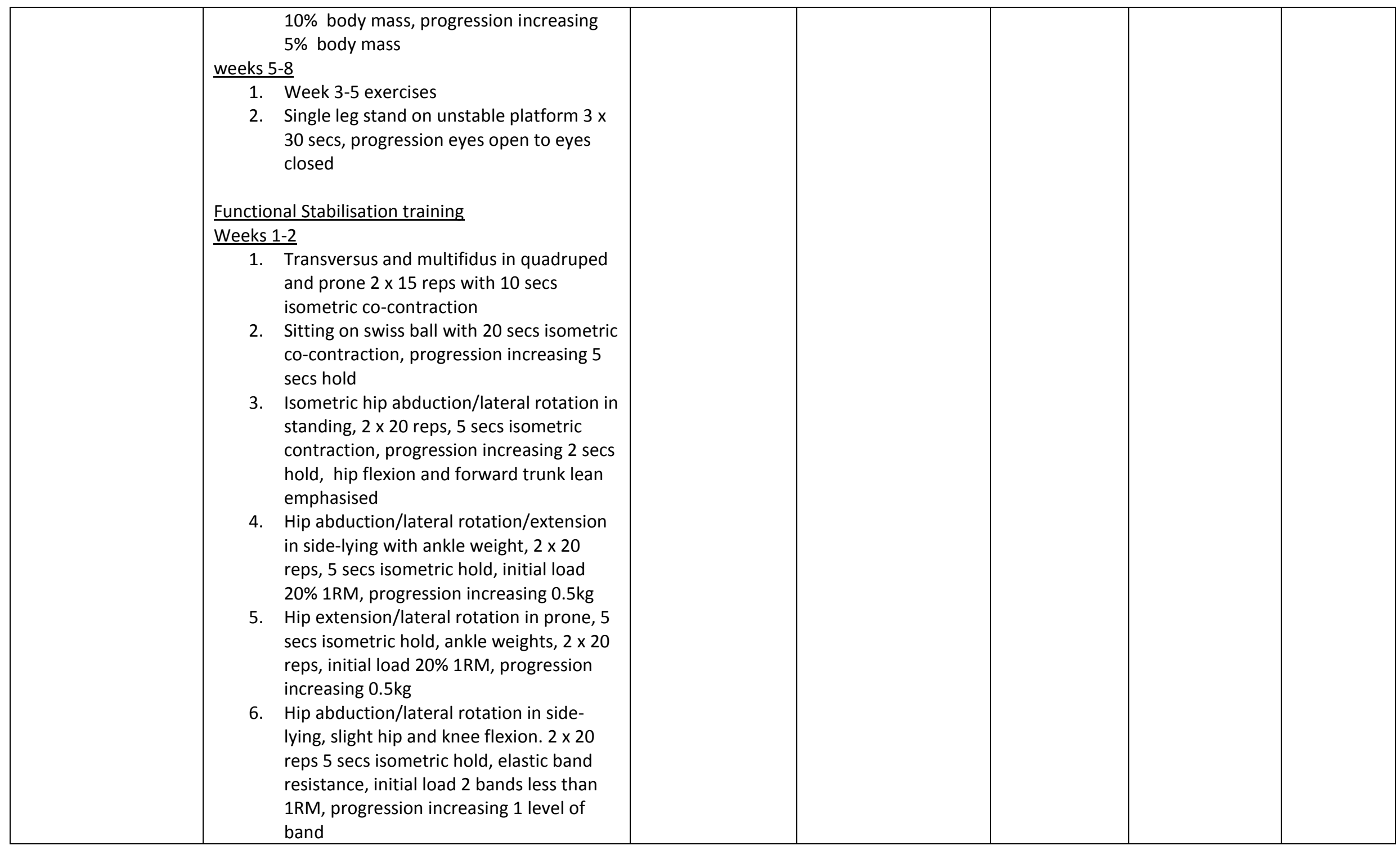




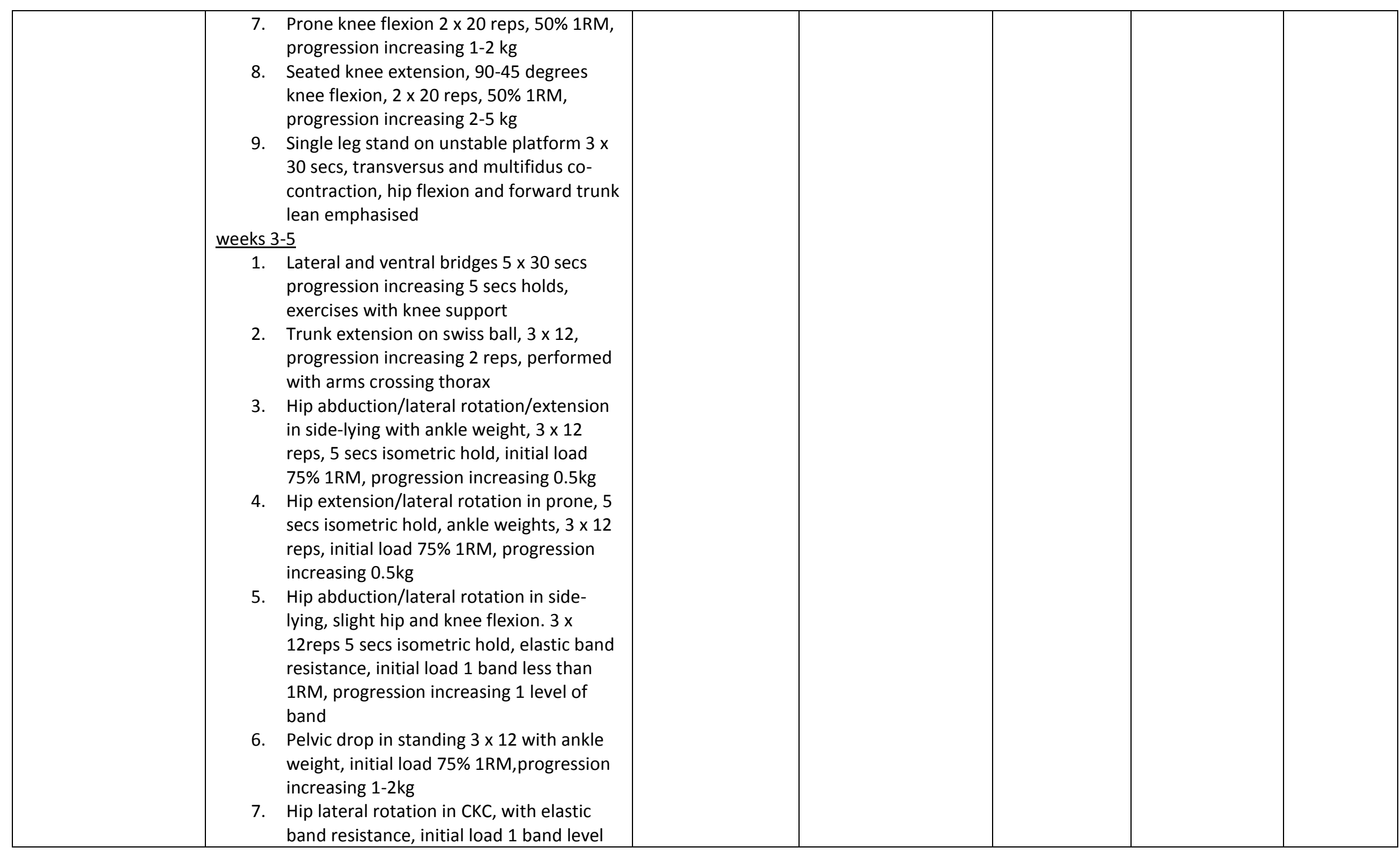




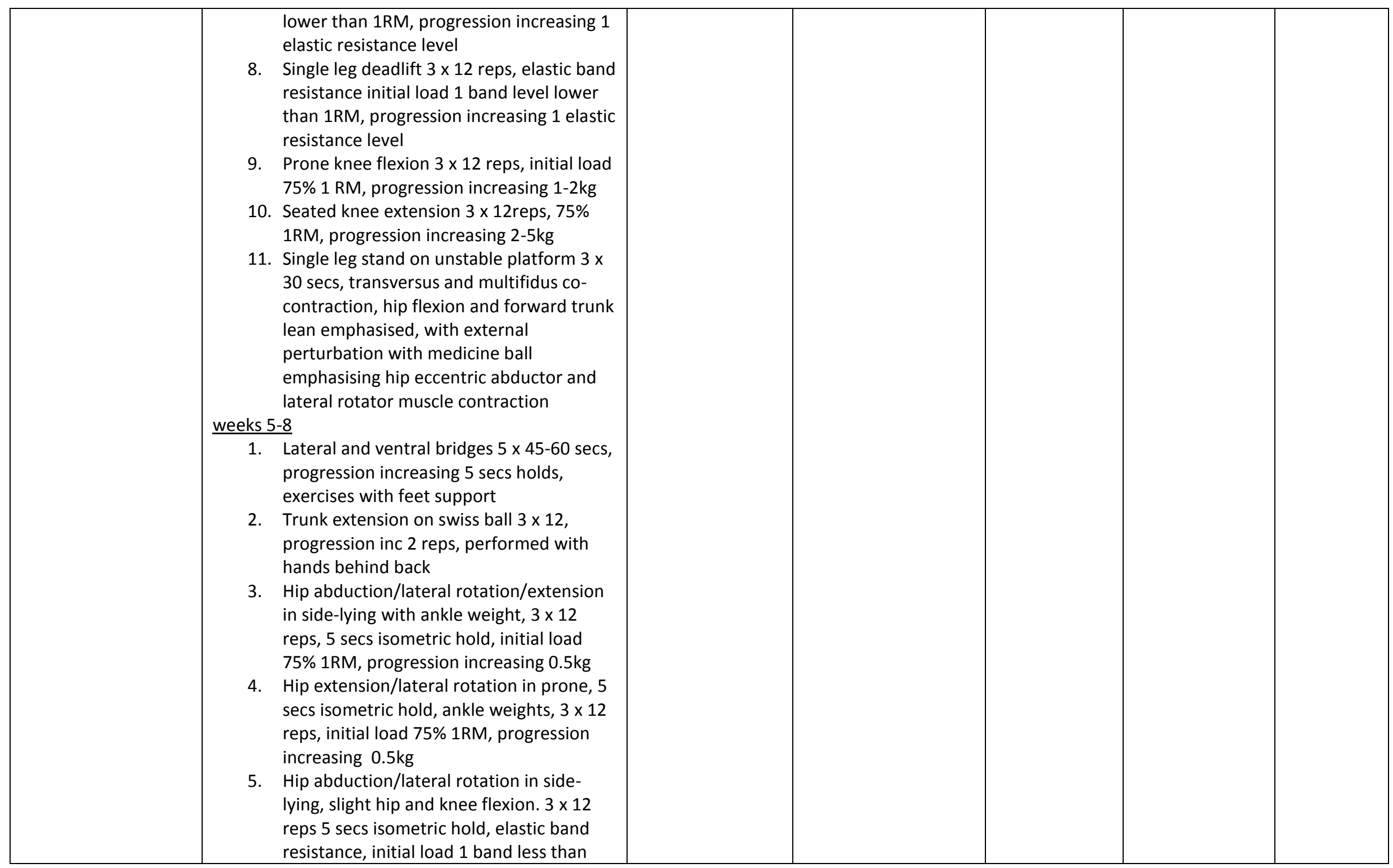




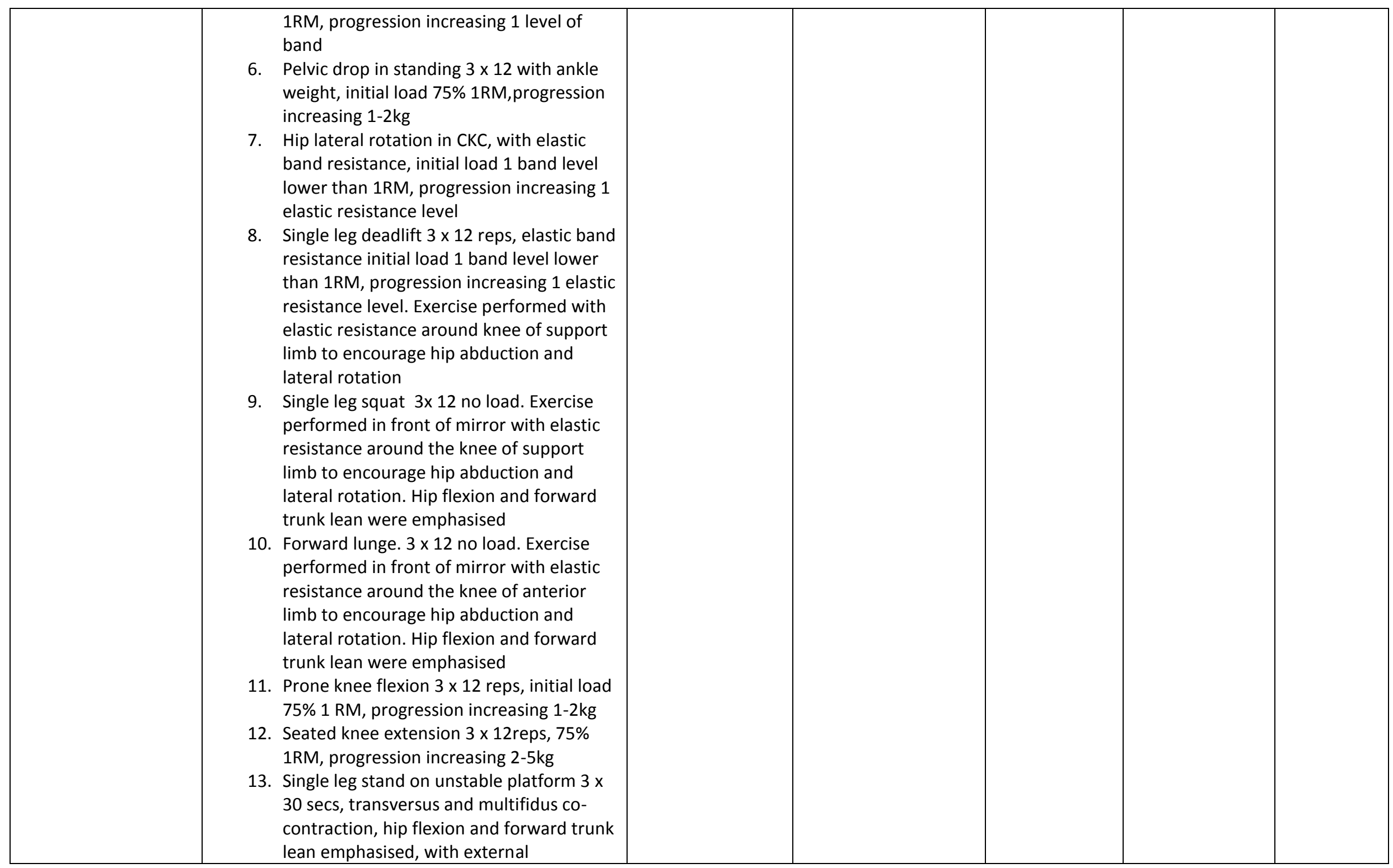




\begin{tabular}{|c|c|c|c|c|c|c|}
\hline & $\begin{array}{l}\text { perturbation with medicine ball } \\
\text { emphasising hip eccentric abductor and } \\
\text { lateral rotator muscle contraction }\end{array}$ & & & & & \\
\hline Coppack et al 2011 & $\begin{array}{ll}\text { Prevention group } \\
\text { 1. } \\
\text { Isometric hip abduction against wall in } \\
\text { standing } \\
\text { 2. Forward lunges } \\
\text { 3. Single leg step-downs from } 20 \mathrm{~cm} \text { step } \\
\text { 4. Single leg squats to } 45 \text { degrees knee } \\
\text { flexion with isometric gluteal contraction } \\
\text { Control group } \\
\text { 1. Slow running } \\
\text { 2. General upper and lower body stretching } \\
\text { 3. Abdominal curls } \\
\text { 4. Push-up drills }\end{array}$ & $\begin{array}{l}\text { Criteria- time } \\
\text { at week } 7 \text { and } 13 \\
\text { increase in reps }\end{array}$ & $\begin{array}{l}\text { Quadriceps, ITB, } \\
\text { hamstring, calf } \\
\text { stretches }\end{array}$ & 14 weeks & $\begin{array}{l}7 \text { x week } \\
1 . \underline{\text { Hip }} \\
\text { abduction. } \\
\text { weeks 1-6 x10 } \\
\text { reps } \\
\text { weeks 7-12 } \\
\text { x15 reps } \\
\text { weeks 13-14 } \\
\text { x15 reps } \\
2 . \text { lunges } \\
\text { weeks 1-6 x10 } \\
\text { reps } \\
\text { weeks 7-12 } \\
\text { x12 reps } \\
\text { weeks 13-14 } \\
\text { x14 reps } \\
\text { 3.step-downs } \\
\text { weeks 1-6 x10 } \\
\text { reps } \\
\text { weeks 7-12 } \\
\text { x12 reps } \\
\text { weeks 13-14 } \\
\text { x14 reps } \\
4 . \text { single leg } \\
\text { squats } \\
\text { weeks 1-6 x10 } \\
\text { reps } \\
\text { weeks 7-12 } \\
\text { x12 reps } \\
\text { weeks 13-14 }\end{array}$ & 14 weeks \\
\hline
\end{tabular}




\begin{tabular}{|c|c|c|c|c|c|c|}
\hline & & & & & x14 reps & \\
\hline Dolak et al 2011 & 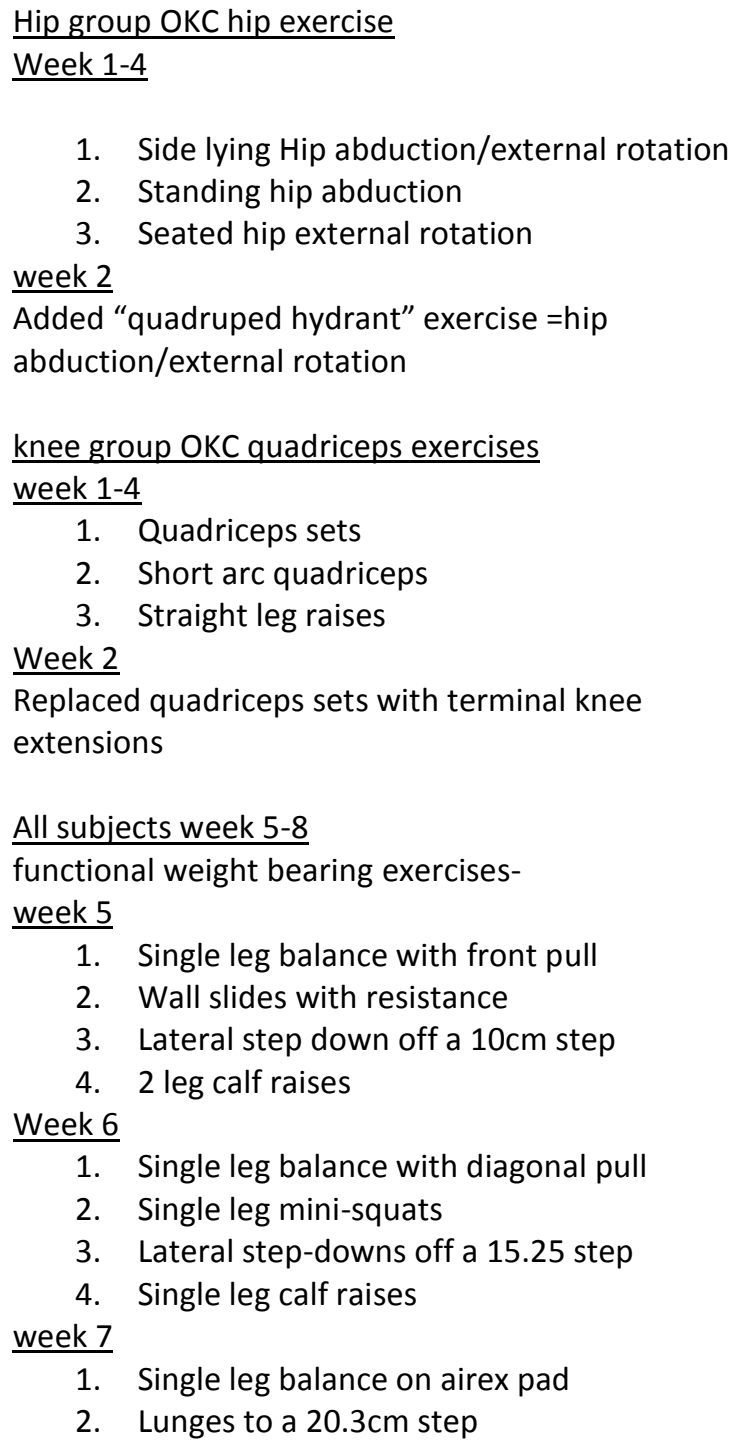 & $\begin{array}{l}\frac{\text { Criteria- time }}{\text { Weeks } 1-4} \\
\text { increasing } \% \text { body } \\
\text { weight } \\
\text { from week } 2-4 \\
(3 \% / 5 \% / 7 \%) \\
\frac{\text { Weeks } 5-8}{\text { increasing }} \\
\text { demand of } \\
\text { exercise }\end{array}$ & $\begin{array}{l}\text { Hamstring (in sitting), } \\
\text { Quadriceps } \\
\text { (standing), TA } \\
\text { (against } \\
\text { wall)stretches }\end{array}$ & 8 weeks & $\begin{array}{l}3 \times 10 \text { reps } \\
3 \times \text { week } \\
\text { ( } 1 \text { supervised, } 2 \\
\text { home) }\end{array}$ & 8 weeks \\
\hline
\end{tabular}




\begin{tabular}{|c|c|c|c|c|c|}
\hline & $\begin{aligned} & \text { 3. } \text { Lateral step-downs off a } 15.25 \mathrm{~cm} \text { step } \\
& \text { with resistance } \\
& \text { 4. }\end{aligned}$ & & & & \\
\hline
\end{tabular}




\begin{tabular}{|c|c|c|c|c|c|c|}
\hline & 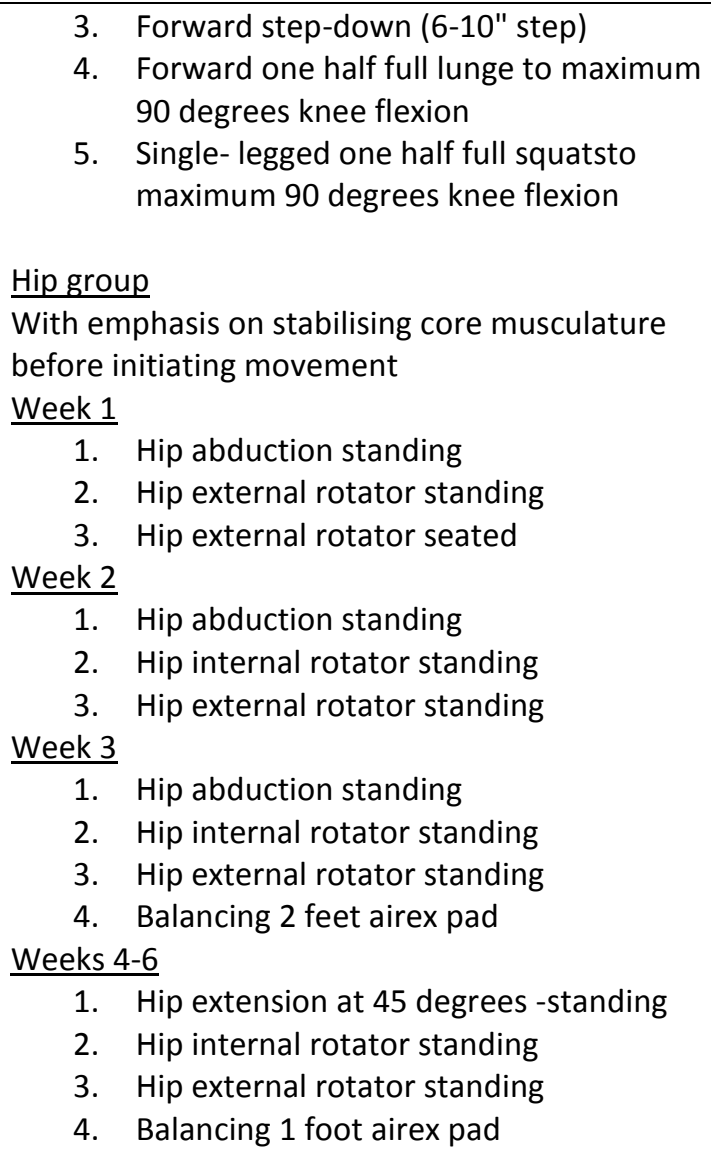 & & & & $\begin{array}{l}3 \times 15 \text { reps } \\
\text { Double legged } \\
\text { wall squats } 3 \times \\
30-45 \text { secs } \\
\frac{\text { Hip group }}{\text { Weeks 1-3 }} \\
3 \text { sets of } 10 \text { reps } \\
\text { Balance } 3 \times 30- \\
45 \text { secs } \\
\frac{\text { Week } 3}{\text { stronger }} \\
\text { theraband } \\
\frac{\text { Weeks } 4-6}{3 \text { sets of } 10-15} \\
\text { reps } \\
\text { Balance } 3 \times 45- \\
60 \text { secs }\end{array}$ & \\
\hline Fukuda et al 2010 & $\begin{array}{ll}\text { Both knee and hip groups- } \\
1 . & \text { Iliopsoas strengthening non-weight } \\
& \text { bearing } \\
\text { 2. } & \text { Seated knee extension } 90-45 \text { degrees } \\
\text { 3. } & \text { Leg press } 0-45 \text { degrees } \\
\text { 4. } & \text { Squatting } 0-45 \text { degrees }\end{array}$ & $\begin{array}{l}1 \mathrm{RM} \text { and } 10 \mathrm{RM} \\
\text { reviewed weekly }\end{array}$ & $\begin{array}{l}\text { Hamstring, calf, } \\
\text { quadriceps, ITB } \\
\text { therapist assisted } \\
\text { stretches }\end{array}$ & 4 weeks & $\begin{array}{l}3 \times 10 \text { reps, } \\
\text { Load } 70 \% 1 \mathrm{RM} \\
\text { or } \\
10 \mathrm{RM} \text { with } \\
\text { theraband } \\
3 \times \text { week }\end{array}$ & 4 weeks \\
\hline
\end{tabular}




\begin{tabular}{|c|c|c|c|c|c|c|}
\hline & $\begin{array}{l}\text { Hip group- in addition } \\
\text { 1. Hip abduction in standing with theraband } \\
\text { 2. Hip abduction side-lying plus weight } \\
\text { 3. Hip external rotation in sitting with } \\
\text { theraband } \\
\text { 4. Side step with theraband in standing } 3 \times \\
\text { 1minute }\end{array}$ & & & & & \\
\hline Fukuda et al 2012 & $\begin{array}{ll}\text { Knee and hip groups } \\
\text { 1. } & \text { Seated knee extension } 90-45 \text { degrees } \\
\text { 2. } & \text { Leg press } 0-45 \text { degrees } \\
\text { 3. } & \text { Squats } 0-45 \text { degrees } \\
\text { 4. } & \text { Single leg calf raises } \\
\text { 5. } & \text { Prone knee flexion } \\
\text { Hip group Addition of } \\
\text { 1. Side-lying hip abduction +weight } \\
\text { 2. Standing hip abduction + theraband } \\
\text { 3. Sitting hip external rotation +theraband } \\
\text { 4. } \text { Hip extension machine resistance }\end{array}$ & $\begin{array}{l}1 \mathrm{RM} \text { and } 10 \mathrm{RM} \\
\text { reviewed weekly }\end{array}$ & $\begin{array}{l}\text { Hamstrings, } \\
\text { plantarflexors, } \\
\text { quadriceps, ITB } \\
\text { stretches both } \\
\text { groups }\end{array}$ & 4 weeks & $\begin{array}{l}3 \times 10 \text { reps, } \\
\text { Load } 70 \% 1 \mathrm{RM} \\
\text { or } \\
10 \mathrm{RM} \text { with } \\
\text { theraband } \\
3 \times \text { week }\end{array}$ & $\begin{array}{l}3 \text { weeks, } 6 \\
\text { months, } 12 \\
\text { months } \\
\text { post } \\
\text { treatment }\end{array}$ \\
\hline Ismail et al 2013 & $\begin{aligned} & \text { Both groups CKC exercise } \\
& \text { 1. } \text { Mini wall squat } \\
& \text { 2. } \text { Forward step-up } \\
& \text { 3. } \text { Lateral step-up } \\
& \text { 4. } \text { Knee extension in standing with } \\
& \text { theraband resistance } \\
& \text { Group } 2 \text { additional hip OKC } \\
& \text { 1. } \\
& \text { 2. Side-lying hip abduction } \\
& \text { Seated external rotation }\end{aligned}$ & $\begin{array}{l}\text { New 10RM } \\
\text { calculated weekly }\end{array}$ & $\begin{array}{l}\text { LL stretches both } \\
\text { groups }\end{array}$ & 6 weeks & $\begin{array}{l}2 \times 10 \text { reps } \\
60 \% 10 \mathrm{RM} \\
3 \times \text { week }\end{array}$ & 6 weeks \\
\hline $\begin{array}{l}\text { Khayambashi et al } \\
2012\end{array}$ & $\begin{array}{l}\text { Control group } \\
\text { no exercise } \\
\text { Exercise group }\end{array}$ & $\begin{array}{l}\text { Theraband(reps) } \\
\text { both legs } \\
\text { Week 1-2 } \\
\text { red(20) } \\
\text { green(20) } \\
\text { blue (20) }\end{array}$ & & 8 weeks & $\begin{array}{l}3 \times 20 \text { reps } \\
3 \times \text { week } \\
\text { (supervised) }\end{array}$ & $\begin{array}{l}8 \text { weeks } \\
6 \text { months } \\
\text { (exercise } \\
\text { group) }\end{array}$ \\
\hline
\end{tabular}




\begin{tabular}{|c|c|c|c|c|c|}
\hline & $\begin{array}{l}\text { 1. Hip abduction +theraband in standing } 0 \text { - } \\
30 \text { degrees } \\
\text { 2. Hip external rotation in sitting + } \\
\text { theraband } 0-30 \text { degrees }\end{array}$ & $\begin{array}{l}\frac{\text { Week 3-4 }}{\text { red (25) }} \\
\text { green (25) } \\
\text { blue (25) } \\
\text { Week 5-6 } \\
\text { green (20) } \\
\text { blue(20) } \\
\text { black (20) } \\
\text { Week 7-8 } \\
\text { green (25) } \\
\text { blue(25) } \\
\text { black (25) }\end{array}$ & & & \\
\hline Nakagawa et al 2008 & 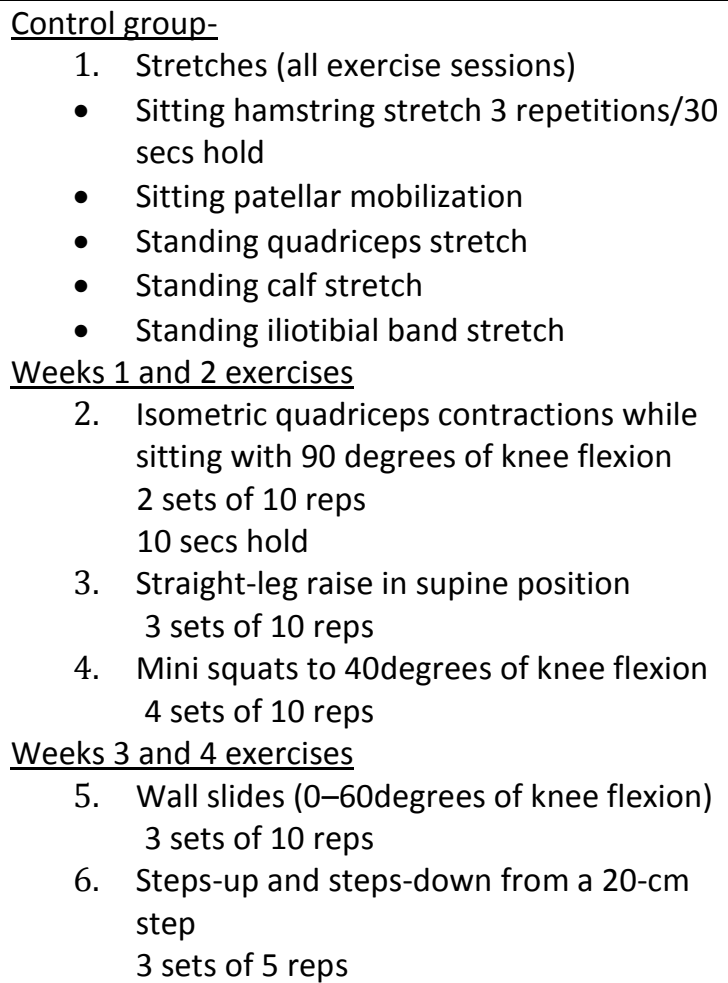 & $\begin{array}{l}\text { Criteria- time, } \\
\text { every } 2 \text { weeks. } \\
\text { Increasing } \\
\text { demand of } \\
\text { exercise, addition } \\
\text { of theraband }\end{array}$ & 6 weeks & $\begin{array}{l}2 \times 15 \text { reps } \\
5 \times \text { week }\end{array}$ & 6 weeks \\
\hline
\end{tabular}




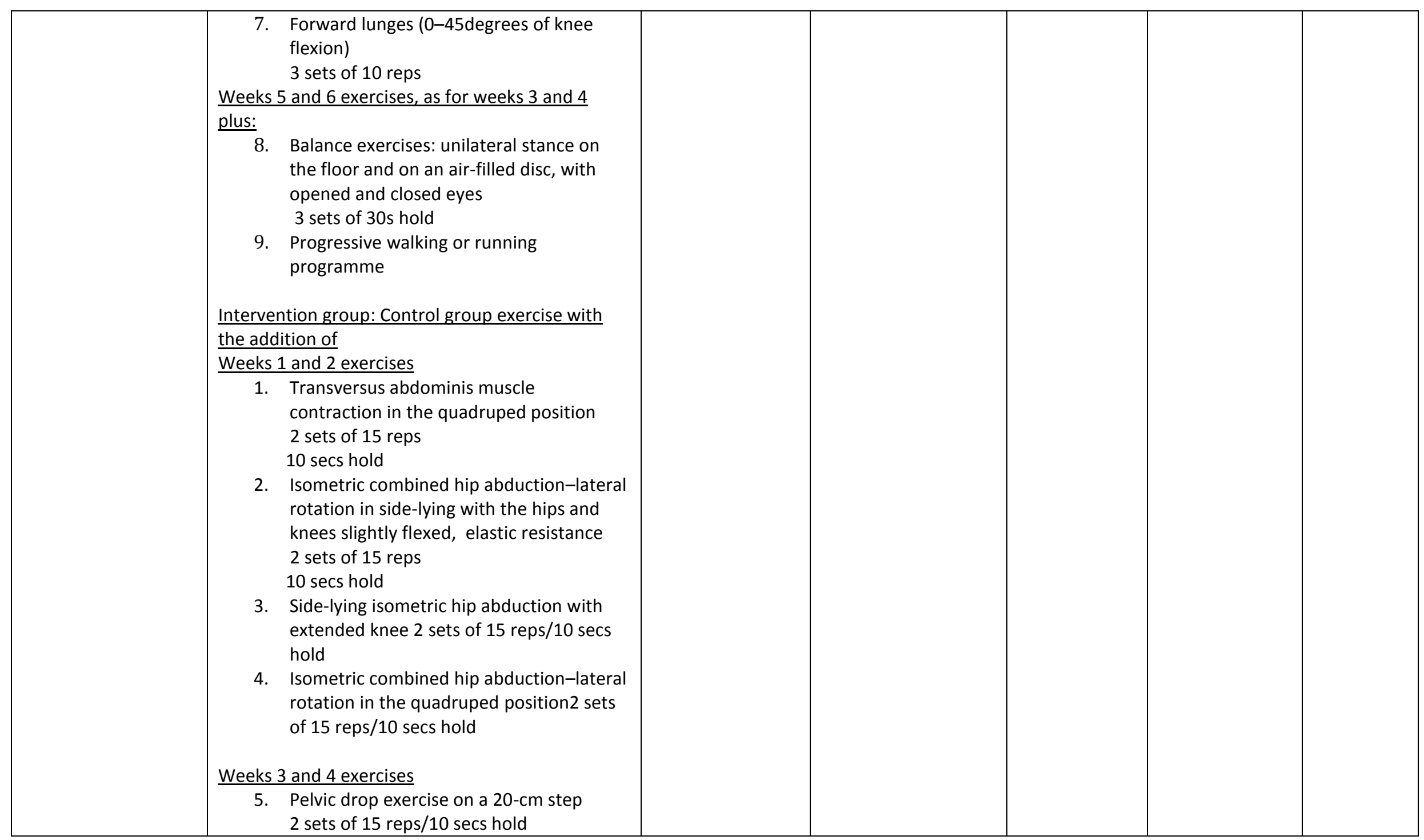




\begin{tabular}{|c|c|c|c|c|c|c|}
\hline & $\begin{array}{l}\text { 6. Upper extremity extension of the } \\
\text { contralateral arm with elastic resistance } \\
\text { performed in single-leg stance } \\
3 \text { sets of } 10 \text { reps } \\
\text { 7. Rotation of the body in the direction of } \\
\text { the contralateral side, holding an elastic } \\
\text { resistance with the ipsilateral arm while } \\
\text { maintaining the lower extremity static } \\
2 \text { sets of } 15 \text { reps } \\
10 \text { secs hold } \\
\text { Weeks } 5 \text { and } 6 \text { exercises, as for weeks } 3 \text { and } 4 \\
\text { Additional elastic resistance around the affected } \\
\text { leg in the forward lunges to encourage lateral } \\
\text { rotation and abduction of the hip }\end{array}$ & & & & & \\
\hline Razeghi et al 2010 & $\begin{array}{l}\text { Knee group } \\
1 . \quad \text { Mini squats } \\
2 . \quad 90-50 \text { degrees resisted knee extension } \\
\text { 3. } \\
\text { Terminal knee extension } \\
\text { Hip and knee group } \\
\text { Bilateral symptoms } \\
\begin{array}{ll}\text { 1. } & \text { All hip muscles and knee extensor } \\
\text { muscles. Details not provided. }\end{array} \\
\begin{array}{ll}\text { Unilateral symptoms } \\
\text { 1. Hip muscle group if strength deficit } \\
\text { detected compared to unaffected limb } \\
\text { and knee extensor muscles. Details not } \\
\text { provided. }\end{array}\end{array}$ & No data & & 4 weeks & No data & 4 weeks \\
\hline Song et al 2009 & $\begin{array}{l}\text { Group 1. } \\
\text { Unilateral leg press } 45-0 \text { degrees knee extension } \\
\text { Group 2. }\end{array}$ & $\begin{array}{l}\text { Exercise at } 60 \% \\
1 \text { RM. } \\
\text { Re-measured and } \\
\text { adjusted every } 2 \\
\text { weeks }\end{array}$ & $\begin{array}{l}\text { Exercise groups } \\
\text { Quadriceps, } \\
\text { hamstring, ITB, calf } \\
\text { stretches }\end{array}$ & 8 weeks & $\begin{array}{l}5 \times 10 \text { reps } \\
60 \% 1 \text { RM } \\
3 \times \text { week }\end{array}$ & 8 weeks \\
\hline
\end{tabular}




\begin{tabular}{|c|c|c|c|c|c|c|}
\hline & $\begin{array}{l}\text { Unilateral leg press } 45-0 \text { degrees knee extension } \\
\text { plus theraband abduction force of } 50 \mathrm{~N} \\
\text { Control group No exercise }\end{array}$ & & & & & \\
\hline
\end{tabular}




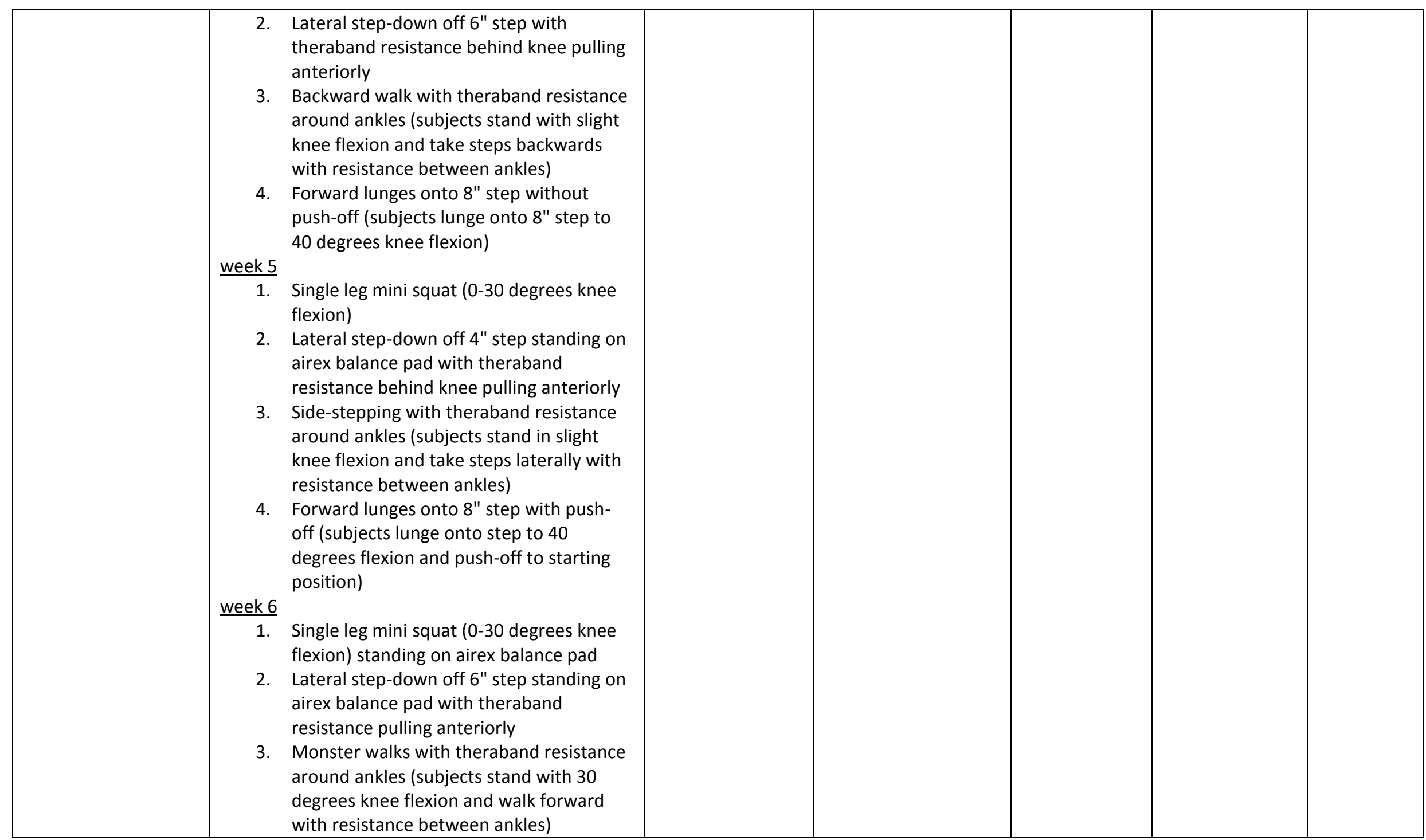




\begin{tabular}{|c|c|c|c|c|c|c|}
\hline & $\begin{array}{l}\text { 4. forward lunges to ground level (subjects } \\
\text { lunge on level surface to } 40 \text { degrees knee } \\
\text { flexion) }\end{array}$ & & & & & \\
\hline Earl and Hoch 2011 & $\begin{array}{l}\text { Phase } 1 \text { (weeks 1-2) } \\
\text { Abdominal exercise and OKC abduction } \\
\text { GOAL- improve volitional control of hip and core } \\
\text { muscles } \\
\text { 1. Abdominal draw in exercises } \\
\text { 2. Side-lying clamshells } \\
\text { 3. Side-lying SLR } \\
\text { 4. Supine arm/leg extensions } \\
\text { 5. Quadruped arm/leg extensions } \\
\text { 6. Isometric single leg stance } \\
\text { 7. Hamstring stretch } \\
\text { 8. Quadriceps stretch } \\
\text { 9. Calf stretch } \\
\text { Phase 2 (weeks 3-5) } \\
\text { OKC abduction with load, CKC functional exercise, } \\
\text { increasing demand of abdominal exercise } \\
\text { GOAL- restore reflex contractions to perturbations } \\
\text { 1. Isometric single leg stand with hip } \\
\text { abduction } \\
\text { 2. Single leg cable column exercise } \\
\quad \text { - Facing towards weights } \\
\quad \text { - Facing away from weights } \\
\text { 3. Single leg stand quick kicks } \\
\text { 4. Prone plank exercises } \\
\text { 5. Side plank exercises } \\
\text { 6. Bilateral mini squat } \\
\text { 7. Hamstring stretch } \\
\text { 8. Quadriceps stretch } \\
\text { 9. Calf stretch }\end{array}$ & $\begin{array}{l}\begin{array}{l}\text { Progression based } \\
\text { on ability to } \\
\text { perform exercise. }\end{array} \\
\text { Dynamic exercise } \\
3 \times 10 \text { reps } \\
3 \times 15 \text { reps } \\
3 \times 20 \text { reps } \\
\text { Isometric exercise } \\
2 \times 15 \text { reps } 10 \text { sec } \\
\text { hold-progress by } \\
\text { adding weight } \\
\text { (2.5-5lb) or } \\
\text { resistance(up one } \\
\text { level/colour } \\
\text { theraband) }\end{array}$ & LL stretches & 8 weeks & $\begin{array}{l}3 \times \text { week } \\
\text { plus } 8-15 \\
\text { supervised } \\
\text { rehabilitation } \\
\text { sessions over } 8 \\
\text { week period }\end{array}$ & 8 weeks \\
\hline
\end{tabular}




\begin{tabular}{|c|c|c|c|c|c|}
\hline & $\begin{array}{l}\text { 10. ITB "pretzel" stretch } \\
\text { Phase } 3 \text { (weeks 6-8) } \\
\text { CKC functional exercise with load or increasing } \\
\text { demand } \\
\text { GOAL- restore pattern generated movements } \\
\text { 1. Monster walks } \\
\text { 2. Single leg stand with sports specific upper } \\
\text { body movement } \\
\text { 3. Mini-squat progression (mini-lunge- } \\
\text { single leg stand- step-down) } \\
\text { 5. Hamstring stretch } \\
\text { 6. Calf stretch stretch } \\
\text { 7. ITB “pretzel" stretch }\end{array}$ & & & & \\
\hline Ferber et al 2011 & $\begin{array}{ll}\text { Exercise } & \text { group } \\
\text { 1. } & \text { Hip abduction +theraband in standing } \\
\text { 2. Hip abduction/extension + theraband in } \\
\text { standing } 45 \text { degree angle } \\
\text { extension/abduction } \\
\text { Control group- no exercise }\end{array}$ & $\begin{array}{l}\text { Reviewed after } 7- \\
10 \text { days } \\
\text { Increased } \\
\text { theraband } \\
\text { resistance if too } \\
\text { easy }\end{array}$ & 3 weeks & $\begin{array}{l}3 \times 10 \text { reps both } \\
\text { legs } \\
\text { daily }\end{array}$ & 3 weeks \\
\hline $\begin{array}{l}\text { Khayambashi et al } \\
2014\end{array}$ & $\begin{array}{l}\text { Hip exercise group } \\
\text { 1. OKC hip abduction 0-30 degrees against } \\
\text { theraband resistance in side-lying } \\
\text { 2. OKC hip external rotation 0-30 degrees } \\
\text { against theraband resistance in sitting, } \\
\text { knee flexion } 90 \text { degrees } \\
\text { Quadriceps exercise group }\end{array}$ & $\begin{array}{l}\text { Progressed every } \\
2 \text { weeks } \\
\text { Theraband } \\
\text { colour/resistance } \\
\text { (reps) } \\
\text { Week 1-2 } \\
\text { red (20) } \\
\text { green (20) } \\
\text { blue (20) } \\
\frac{\text { Week 3-4 }}{\text { red (25) }}\end{array}$ & 8 weeks & $\begin{array}{l}3 \times 20 / 25 \text { reps } \\
3 \times \text { week } \\
\text { (supervised) } \\
\text { Exercises } \\
\text { bilaterally in } \\
\text { bilateral PFP, } \\
\text { unilaterally in } \\
\text { unilateral PFP }\end{array}$ & 8 weeks \\
\hline
\end{tabular}




\begin{tabular}{|c|c|c|c|c|c|c|}
\hline & $\begin{array}{l}\text { 1. OKC knee extension from } 30 \text { degrees } \\
\text { flexion to full extension, in sitting, against } \\
\text { theraband resistance } \\
\text { 2. Partial squatting } 30 \text { degrees flexion to full } \\
\text { extension against theraband resistance, } \\
\text { while squeezing ball between knees }\end{array}$ & $\begin{array}{l}\text { green }(25) \\
\text { blue }(25) \\
\text { Week 5-6 } \\
\text { green }(20) \\
\text { blue }(20) \\
\text { black (20) } \\
\text { Week 7-8 } \\
\text { green (25) } \\
\text { blue }(25) \\
\text { black (25) }\end{array}$ & & & & \\
\hline Lowry 2008 & $\begin{array}{l}\text { Non-weight bearing exercise } \\
\text { 1. } \begin{array}{l}\text { Isometric abdominal bracing in hook-lying } \\
\text { with heel slide, bent knee lifts and SLR }\end{array} \\
\text { 2. Bridging } \\
\text { 3. Side-lying clamshells } \\
\text { 4. Quadruped hip extension with opposite } \\
\text { shoulder flexion } \\
\text { 5. Quadruped hip abduction/extension } \\
\text { Weight bearing exercise } \\
\text { 1. Double leg press on gym machine } \\
\text { 2. Single leg press on gym machine } \\
\text { 3. Eccentric step-downs from step } \\
\text { 4. Eccentric side step-downs from step } \\
\text { 5. Hip abduction on side-stepping with } \\
\text { theraband resistance } \\
\text { 6. Squats } \\
\text { 7. Lunges } \\
\text { 8. "clock balance and reach" (single leg } \\
\text { stand, reach with opposite heel to 12:00, } \\
\text { and to the side (1:00) and so forth in a } \\
\text { circle }\end{array}$ & $\begin{array}{l}\text { Non weight } \\
\text { bearing exercises } \\
\text { for } 2 \text { weeks until } \\
\text { achieved } 2 \times 10 \\
\text { reps }\end{array}$ & $\begin{array}{l}\text { Stretches } \\
\text { Piriformis } \\
\text { Gluteals } \\
\text { Hamstrings } \\
\text { Quadriceps } \\
\text { ITB } \\
\text { Gastrocnemius/soleu } \\
\text { s } \\
\text { Manual therapy to } \\
\text { lumbar spine, hip, } \\
\text { patellofemoral and } \\
\text { tibiofemoral joints } \\
\text { Taping if relieved } \\
\text { pain on step-down } \\
\text { test } \\
\text { Orthotics if excessive } \\
\text { pronation (defined } \\
>3 \text { mm drop in } \\
\text { functional activity) }\end{array}$ & 6-14 weeks & $\begin{array}{l}\text { Exercises } \\
\text { individualised } \\
\text { per patient, 1-2 } \\
x \text { daily, no } \\
\text { further detail }\end{array}$ & $\begin{array}{l}\text { At } \mathrm{D} / \mathrm{C} \text { and } \\
6 \text { months }\end{array}$ \\
\hline Mascal 2003 & $\begin{array}{l}\text { 1. Non-weight-bearing OKC hip abduction, } \\
\text { gluteus medius and gluteus maximus } \\
\text { exercises }\end{array}$ & $\begin{array}{l}\text { Criteria - ability to } \\
\text { achieve } 2 \times 15 \\
\text { reps or } 10 \text { secs }\end{array}$ & & 14 weeks & $\begin{array}{l}2-3 \times 10-15 \text { reps } \\
2 \times \text { daily }\end{array}$ & 14 weeks \\
\hline
\end{tabular}




\begin{tabular}{|c|c|c|c|c|c|c|}
\hline & $\begin{array}{l}\text { 2. Weight-bearing in single leg stance } \\
\text { 3. Functional exercise }\end{array}$ & $\begin{array}{l}\text { isometric hold. } \\
\text { Progressed to } \\
\text { increasing } \\
\text { demand of } \\
\text { exercise. }\end{array}$ & & & & \\
\hline Tyler 2006 & 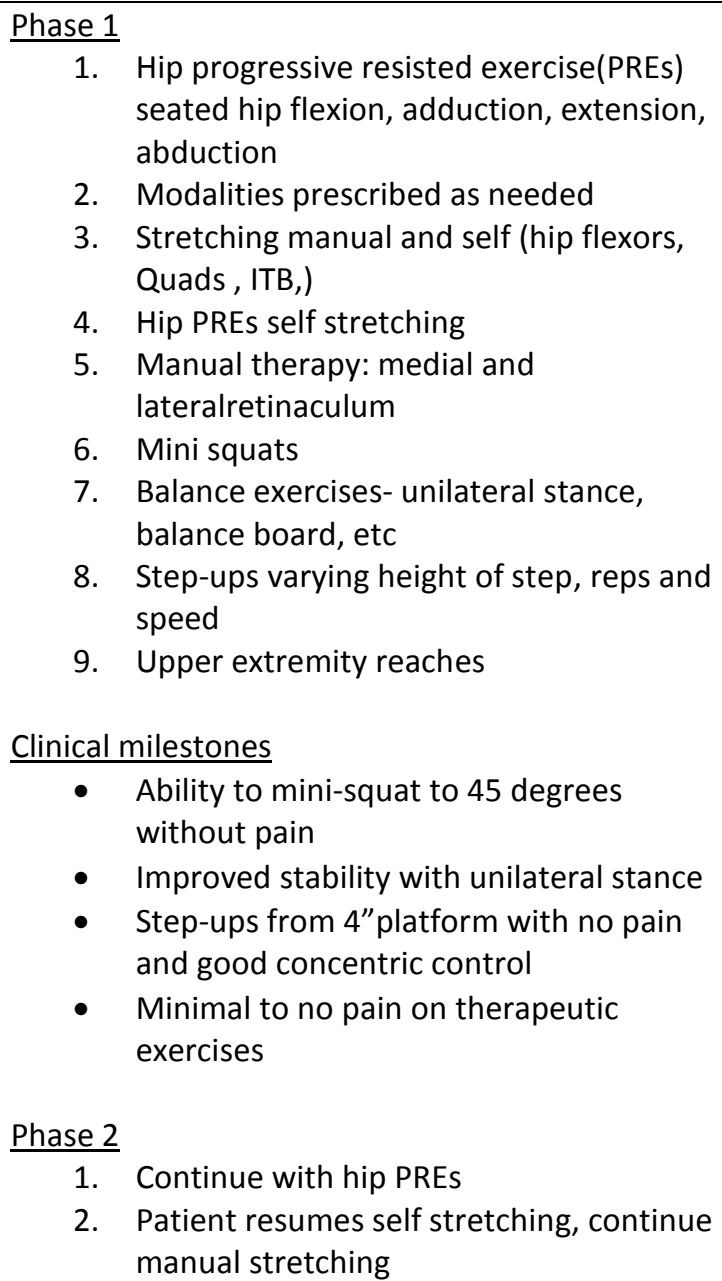 & $\begin{array}{l}\text { Specific criteria } \\
\text { for progression } \\
\text { based on ability } \\
\text { to perform } \\
\text { exercises }\end{array}$ & $\begin{array}{l}\text { Manual therapy to } \\
\text { PFJ } \\
\text { Manual and self LL } \\
\text { stretches }\end{array}$ & 6 weeks & $\begin{array}{l}\text { Daily, no further } \\
\text { information }\end{array}$ & 6 weeks \\
\hline
\end{tabular}




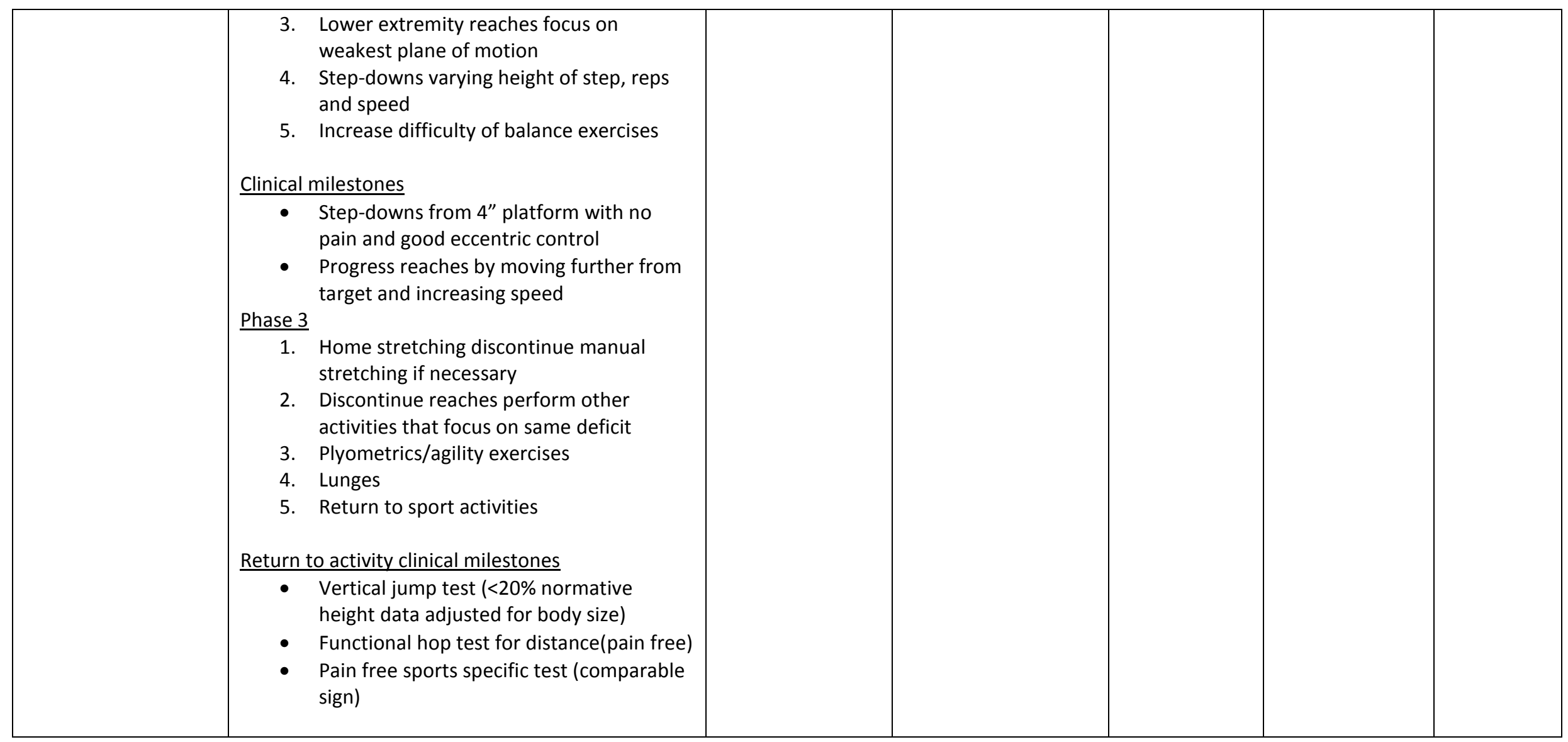

Key:

OKC- open kinetic chain

CKC- closed kinetic chain

SLR- straight leg raise

RM- repetition maximum 
PRE - progressive resisted exercise

Reps- repetitions

Secs - seconds

LL- lower limb

PFJ-patellofemoral joint

Table 8 Exercises known to recruit Gluteal muscles(Reiman et al., 2011).

\begin{tabular}{|c|c|c|c|c|c|c|c|c|c|c|c|c|}
\hline & \multicolumn{2}{|c|}{$\begin{array}{l}\text { Very high activation } \\
\text { Gluteus maximus }\end{array}$} & \multicolumn{2}{|c|}{$\begin{array}{l}\text { High activation } \\
\text { Gluteus maximus }\end{array}$} & \multicolumn{2}{|c|}{$\begin{array}{l}\text { Moderate activation } \\
\text { Gluteus maximus }\end{array}$} & \multicolumn{2}{|c|}{$\begin{array}{l}\text { Very high activation } \\
\text { Gluteus medius }\end{array}$} & \multicolumn{2}{|c|}{$\begin{array}{l}\text { High activation } \\
\text { Gluteus medius }\end{array}$} & \multicolumn{2}{|c|}{$\begin{array}{l}\text { Moderate } \\
\text { activation Gluteus } \\
\text { medius }\end{array}$} \\
\hline & $\begin{array}{l}\begin{array}{l}\text { Proximal } \\
\text { exercise }\end{array} \\
\end{array}$ & $\begin{array}{l}\text { Control } \\
\text { Group }\end{array}$ & $\begin{array}{c}\text { Proximal } \\
\text { exercise }\end{array}$ & $\begin{array}{l}\text { Control } \\
\text { Group }\end{array}$ & $\begin{array}{l}\text { Proximal } \\
\text { exercise }\end{array}$ & $\begin{array}{l}\text { Control } \\
\text { Group }\end{array}$ & $\begin{array}{c}\text { Proximal } \\
\text { exercise }\end{array}$ & $\begin{array}{l}\text { Control } \\
\text { Group }\end{array}$ & $\begin{array}{l}\text { Proximal } \\
\text { exercise }\end{array}$ & $\begin{array}{l}\text { Control } \\
\text { Group }\end{array}$ & $\begin{array}{l}\text { Proximal } \\
\text { exercise }\end{array}$ & $\begin{array}{l}\text { Control } \\
\text { Group }\end{array}$ \\
\hline $\begin{array}{l}\text { Avraham et al., } \\
2007\end{array}$ & & & SLSq & SLSq & & & SLSq & SLSq & & & & \\
\hline Baldon et al., 2014 & & Step-up & $\begin{array}{l}\text { Single leg } \\
\text { deadlift }\end{array}$ & $\begin{array}{l}\text { Wall- } \\
\text { squat } \\
\text { Step-down }\end{array}$ & $\begin{array}{l}\text { Forward } \\
\text { lunge }\end{array}$ & & $\begin{array}{l}\text { Lateral } \\
\text { bridge }\end{array}$ & & $\begin{array}{l}\text { Pelvic drop } \\
\text { Single leg } \\
\text { deadlift }\end{array}$ & $\begin{array}{l}\text { Step-up } \\
\text { Wall-squat }\end{array}$ & $\begin{array}{l}\text { Forward } \\
\text { lunge }\end{array}$ & \\
\hline Boling et al., 2006 & & $\mathrm{~N} / \mathrm{C}$ & $\begin{array}{l}\text { Wall slides } \\
\text { Lateral } \\
\text { step-down } \\
\text { SLSq }\end{array}$ & & $\begin{array}{l}\text { Forward } \\
\text { lunge }\end{array}$ & & SLSq & & $\begin{array}{l}\text { Wall slide } \\
\text { Lateral step- } \\
\text { down }\end{array}$ & & $\begin{array}{l}\text { Forward } \\
\text { lunge }\end{array}$ & \\
\hline $\begin{array}{l}\text { Coppack et al., } \\
2011\end{array}$ & & & $\begin{array}{l}\text { SLSq } \\
\text { step-down }\end{array}$ & & & & SLSq & & & & $\begin{array}{l}\text { Forward } \\
\text { lunge } \\
\text { step-down }\end{array}$ & \\
\hline Dolak et al., 2011 & & & $\begin{array}{l}\text { Wall slide } \\
\text { Lateral } \\
\text { step- } \\
\text { down, } \\
\text { SLSq }\end{array}$ & & $\begin{array}{l}\text { Addition } \\
\text { side-ly OKC } \\
\text { abd }\end{array}$ & $\begin{array}{l}\text { Forward } \\
\text { lunge }\end{array}$ & SLSq & & $\begin{array}{l}\text { Wall slide } \\
\text { Lateral step- } \\
\text { down } \\
\text { Addition } \\
\text { side-ly OKC } \\
\text { abd }\end{array}$ & & $\begin{array}{l}\text { Forward } \\
\text { lunge }\end{array}$ & \\
\hline Earl et al., 2011 & & $\mathrm{~N} / \mathrm{C}$ & $\begin{array}{l}\text { SLSq } \\
\text { Quad }\end{array}$ & & $\begin{array}{l}\text { Clam } \\
\text { Side-ly OKC } \\
\text { abd }\end{array}$ & & SLSq & & $\begin{array}{l}\text { Side-ly OKC } \\
\text { abd, } \\
\text { Quad }\end{array}$ & & $\begin{array}{l}\text { Prone- } \\
\text { plank, } \\
\text { clam }\end{array}$ & \\
\hline Ferber et al., 2011 & & $\mathrm{~N} / \mathrm{C}$ & & & & & & & & & & \\
\hline
\end{tabular}




\begin{tabular}{|c|c|c|c|c|c|c|c|c|c|c|c|c|}
\hline Ferber et al., 2014 & & & & $\begin{array}{l}\text { SLSq, } \\
\text { Lateral } \\
\text { step-down } \\
\text { Wall squat }\end{array}$ & & $\begin{array}{l}\text { Forward } \\
\text { lunge }\end{array}$ & & SLSq & & $\begin{array}{l}\text { Lateral } \\
\text { step-down } \\
\text { Wall squat }\end{array}$ & & $\begin{array}{l}\text { Forward } \\
\text { lunge }\end{array}$ \\
\hline Fukuda et al., 2010 & & & & & $\begin{array}{l}\text { Addition } \\
\text { side-ly OKC } \\
\text { abd }\end{array}$ & & & & $\begin{array}{l}\text { Addition } \\
\text { side-ly OKC } \\
\text { abd }\end{array}$ & & & \\
\hline Fukuda et al., 2012 & & & & & $\begin{array}{l}\text { Addition } \\
\text { side-ly OKC } \\
\text { abd }\end{array}$ & & & & $\begin{array}{l}\text { Addition } \\
\text { side-ly OKC } \\
\text { abd }\end{array}$ & & & \\
\hline Ismail et al., 2013 & $\begin{array}{l}\text { Forward } \\
\text { step-up }\end{array}$ & & $\begin{array}{l}\text { Lateral } \\
\text { step-up }\end{array}$ & & $\begin{array}{l}\text { Addition } \\
\text { side-ly OKC } \\
\text { abd }\end{array}$ & & & & $\begin{array}{l}\text { Forward } \\
\text { step-up } \\
\text { Lateral step- } \\
\text { up }\end{array}$ & $\begin{array}{l}\text { Addition } \\
\text { side-ly OKC } \\
\text { abd }\end{array}$ & & \\
\hline $\begin{array}{l}\text { Khayambashi et } \\
\text { al., } 2012\end{array}$ & & $\mathrm{~N} / \mathrm{C}$ & & & & & & & & & & \\
\hline $\begin{array}{l}\text { Khayambashi et al., } \\
2014\end{array}$ & & & & & $\begin{array}{l}\text { Side-ly OKC } \\
\text { abd }\end{array}$ & & & & & $\begin{array}{l}\text { Side-ly OKC } \\
\text { abd }\end{array}$ & & \\
\hline Lowry et al., 2008 & & $\mathrm{~N} / \mathrm{C}$ & Quad & & $\begin{array}{l}\text { Clam, } \\
\text { forward } \\
\text { lunge } \\
\end{array}$ & & & & Quad & & $\begin{array}{l}\text { Forward } \\
\text { lunge, } \\
\text { clam }\end{array}$ & \\
\hline Mascal et al., 2003 & & $\mathrm{~N} / \mathrm{C}$ & SLSq & & $\begin{array}{l}\text { Side-ly OKC } \\
\text { abd }\end{array}$ & & SLSq & & $\begin{array}{l}\text { Side-ly OKC } \\
\text { abd }\end{array}$ & & & \\
\hline $\begin{array}{l}\text { Nakagawa et al., } \\
2008\end{array}$ & & Step-up & & Wall slide & $\begin{array}{l}\text { Additionside } \\
\text {-ly OKC abd }\end{array}$ & $\begin{array}{l}\text { Forward } \\
\text { lunge }\end{array}$ & & & $\begin{array}{l}\text { Addition } \\
\text { side-ly OKC } \\
\text { abd, pelvic } \\
\text { drop } \\
\end{array}$ & $\begin{array}{l}\text { Step-up } \\
\text { Wall slide }\end{array}$ & & $\begin{array}{l}\text { Forward } \\
\text { lunge }\end{array}$ \\
\hline \multicolumn{13}{|l|}{ Razeghi et al., 2010} \\
\hline Song et al., 2009 & & $\mathrm{~N} / \mathrm{C}$ & & & & & & & & & & \\
\hline Tyler et al., 2006 & Step-up & $\mathrm{N} / \mathrm{C}$ & & & $\begin{array}{l}\text { Forward } \\
\text { lunge }\end{array}$ & & & & Step-down & & $\begin{array}{l}\text { Forward } \\
\text { lunge }\end{array}$ & \\
\hline
\end{tabular}

OKC- open kinetic chain

Quad- Quadruped, contralateral arm/leg lift 
SLSq- Single leg squat

Abd- abduction

Side-ly- side lying

$\mathrm{N} / \mathrm{C}$ - no control exercise group 


\section{Effect of hip strengthening exercise on pain}

All studies demonstrated that hip exercises in PFP resulted in a reduction in pain from baseline values (table 9). Six studies used the visual analogue scale (VAS) for usual or worst pain and achieved the minimally clinically important difference (MCID) in patellofemoral pain of $2 \mathrm{~cm}$ (Crossley et al., 2004).

All control exercise programmes demonstrated a significant improvement in pain. There was superior improvement in the hip group in seven studies, although this did not consistently reach clinical significance.

Pain was significantly reduced in all four studies that investigated the addition of hip exercises to an exercise protocol. Three added OKC hip exercises; all demonstrated a significant reduction in pain in the hip group compared to the control group, with two demonstrating change above the MCID in patellofemoral pain of 1.2 on the numerical pain rating scale (Piva et al., 2009) in the hip group only. All three studies that compared hip and knee exercise demonstrated a significant reduction in pain in both groups which was above VAS MCID levels in two studies, with superior results in the hip group in one which was maintained at six month follow-up. One compared initial OKC hip or knee exercises prior to functional exercises; only the hip group had a significant reduction in pain after the initial exercise. There was no between group difference at the end of the exercise programme.

\section{Effect of hip strengthening exercise on function}

All fourteen studies that evaluated function demonstrated a significant improvement (table 9). Although the majority of exercise programmes demonstrated functional improvement there were superior results for proximal exercise across all protocols. Seven studies used the anterior knee pain 
scale achieving a change in ten points which signifies a clinical difference in PFP (Crossley et al., 2004) and five the lower extremity functional scale achieving a MCID of nine points.

Three of the four studies that investigated the addition of hip exercise to a standard programme used a functional outcome and demonstrated improvement in both groups. All demonstrated a clinically relevant change with proximal exercise which only occurred in one measure in one study for the control exercise.

Studies that compared hip and knee exercise demonstrated a significant improvement in both groups, with superior results in the hip groups, reaching significance in one which was maintained at six month follow-up.

There were greater functional gains with proximal strengthening exercise including instruction on lower limb alignment although both groups reached clinical significance (Baldon et al., 2014).

\section{Effect of hip strengthening exercise on kinematics}

Evidence for improvement in kinematics was conflicting (table 9). One study demonstrated no change; two showed an improvement, but both included instruction on lower limb alignment with the strengthening exercise.

\section{Effect of hip strengthening exercises for the prevention of PFP}

The incidence of PFP after hip strengthening exercise was reduced (table 9). 
Table 9 Outcome measures with strengthening exercise

\begin{tabular}{|c|c|c|c|}
\hline Study & Pain outcome & Functional outcome & Other \\
\hline $\begin{array}{l}\text { Avraham et al } \\
2007\end{array}$ & $\begin{array}{l}\text { VAS (no detail specified) } \\
\text { No numerical data recorded } \\
\text { Bar graphs with p values comparing groups } \\
\text { demonstrated reduction in pain in all groups; } \\
\text { greater reduction in hip and hip \& knee exercise } \\
\text { groups }\end{array}$ & $\begin{array}{l}\text { Patellofemoral evaluation scale } \\
\text { No numerical data recorded } \\
\text { Bar graphs with p values comparing groups demonstrated } \\
\text { improvement in function in all groups; greater reduction } \\
\text { in hip and hip \& knee exercise groups }\end{array}$ & \\
\hline $\begin{array}{l}\text { Baldon et al } \\
2014\end{array}$ & $\begin{array}{l}\text { VAS }(\mathrm{cm})(\text { worst pain previous week) } \\
\text { Both groups less pain at } 8 \text { weeks and } 3 \text { months } \\
p<0.001 \\
\text { FST group less pain than ST group at } 8 \text { weeks } p=0.06 \\
\text { and } 3 \text { months } p=0.04 \\
\text { Standard training (ST): } 6.1+/-1.8 \text { to } 3.1+/-3.2 \text { (8 } \\
\text { weeks) to } 2.5+/-2.7(3 \text { months) } \\
\text { Functional standard training (FST): } 6.6+/-1.1 \text { to } \\
1.4+/-1.4 \text { ( } 8 \text { weeks) to } 0.9+/-1.5(3 \text { months) }\end{array}$ & 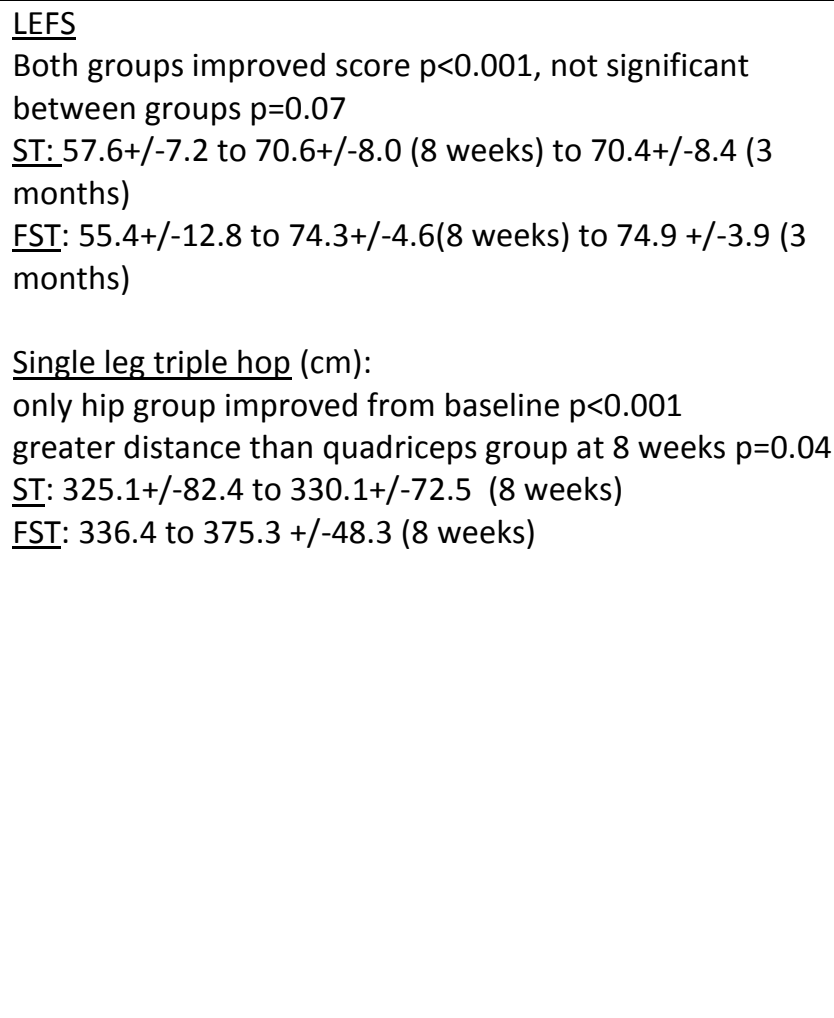 & 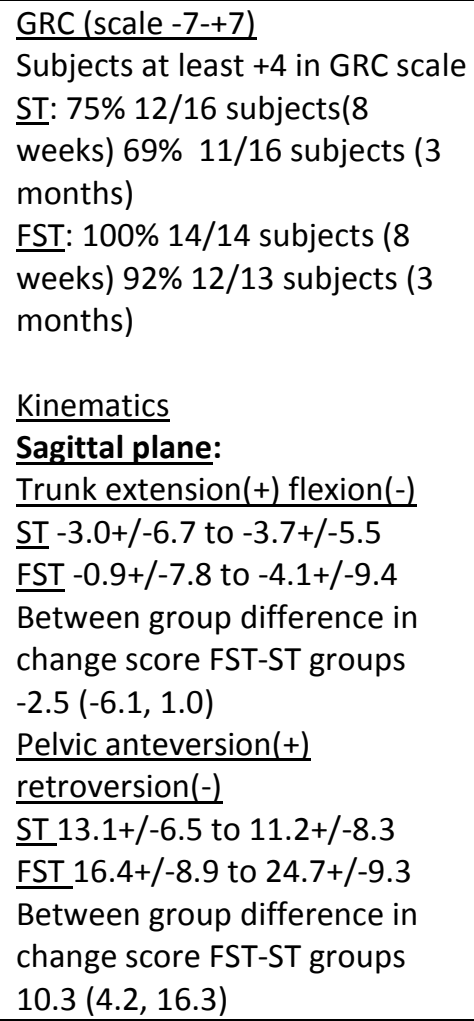 \\
\hline
\end{tabular}




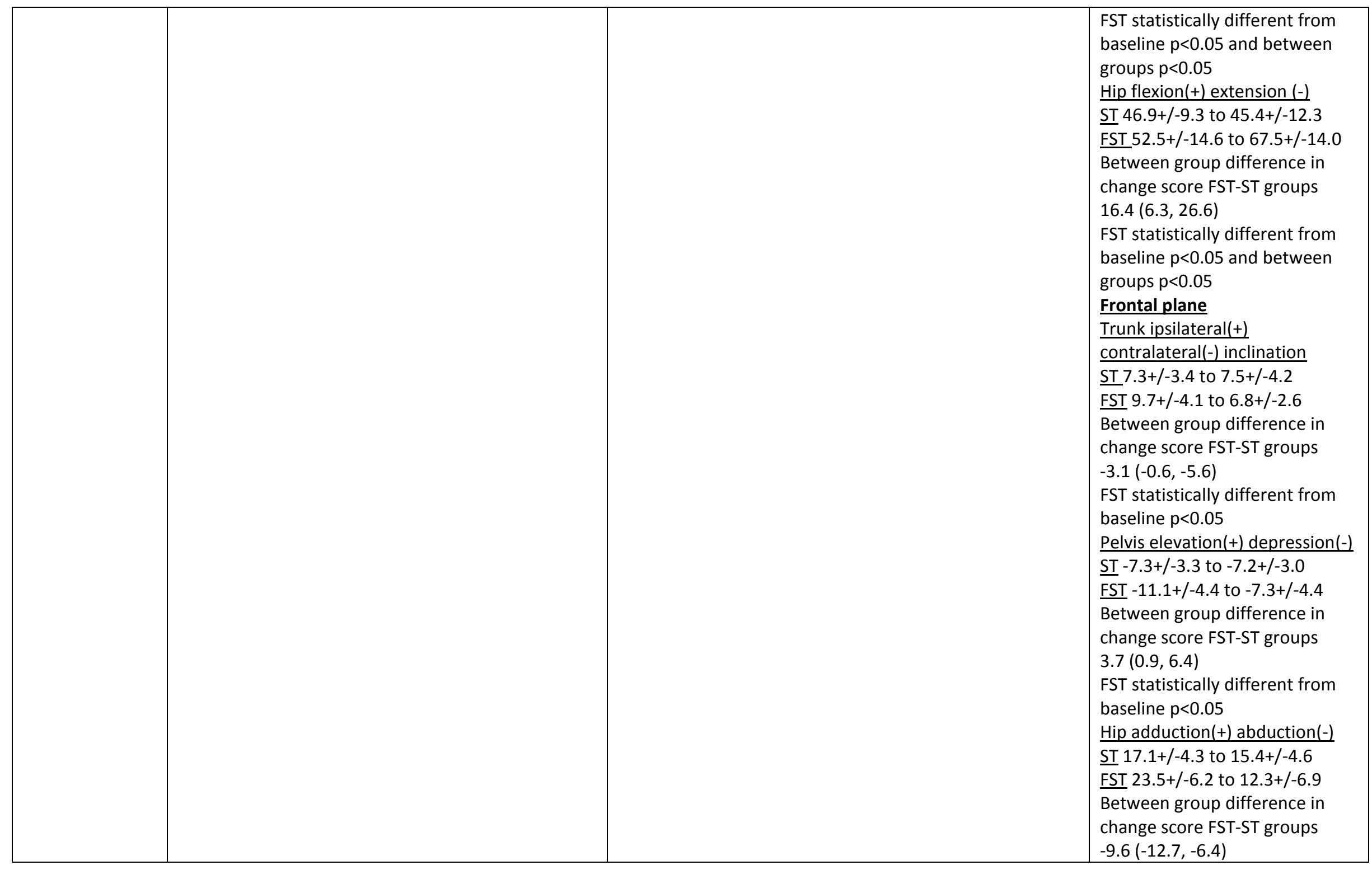




\begin{tabular}{|c|c|c|c|}
\hline & & & $\begin{array}{l}\text { FST statistically different from } \\
\text { baseline } p<0.05 \\
\text { Knee adduction(+) abduction(-) } \\
\text { ST }-11.0+/-7.2 \text { to }-10.9+/-7.4 \\
\text { FST }-12.3+/-5.2 \text { to }-9.0+/-6.3 \\
\text { Between group difference in } \\
\text { change score FST-ST groups } \\
3.3(0.3,6.2) \\
\text { FST statistically different from } \\
\text { baseline } p<0.05\end{array}$ \\
\hline $\begin{array}{l}\text { Coppack et al } \\
2011\end{array}$ & $\mathrm{~N} / \mathrm{A}$ & N/A & $\begin{array}{l}36 \text { new cases of anterior knee } \\
\text { pain in control group }=4.8 \% \\
\text { incidence. } 10 \text { medically } \\
\text { discharged, } 16 \text { completed } \\
\text { training, } 10 \text { other. } \\
10 \text { new cases anterior knee pain } \\
\text { in intervention group }=1.3 \% \\
\text { incidence. } 1 \text { discharged ("unfit } \\
\text { for army service", not medically } \\
\text { discharged) } 9 \text { completed } \\
\text { training. }\end{array}$ \\
\hline Dolak et al 2011 & $\begin{array}{l}\text { VAS }(\mathrm{cm}) 0-10 \text { (worst pain in the last week) } \\
\text { At } 4 \text { weeks Hip group VAS less than knee group } p= \\
0.035 \text { (hip } 2.4+/-2 \text {, knee } 4.1+/-2.5) \\
\text { Hip group baseline } 4.6+/-2.5 \text { significantly less at } 4 \\
\text { weeks }(2.4+/-2) p=0.001 \text {, and at } 8 \text { weeks }(2.4+/-2.8) \\
p=0.003,2.1+/-2.5 \text { at } 3 \text { months } \\
\text { Knee group baseline } 4.2+/-2.3 \text {, not significantly diff } \\
\text { to baseline at } 4 \text { weeks }(4.1+/-2.5) \text {, significantly less } \\
\text { at } 8 \text { weeks }(2.6+/-2.0) \text { than baseline } p=0.028,2.4+/- \\
2.3 \text { at } 3 \text { months }\end{array}$ & $\begin{array}{l}\frac{\text { LEFS- } 0-80}{\text { improved regardless of protocol } p=0.006} \\
\frac{\text { Hip group baseline } 59+/-12,4 \text { weeks } 67+/-11,8 \text { weeks }}{70+/-10,3 \text { months } 70+/-10} \\
\frac{\text { Knee group baseline } 54+/-12,4 \text { weeks } 59+/-14,8 \text { weeks }}{65+/-13,3 \text { months } 67+/-11} \\
\frac{\text { Step down test (no reps in } 30 \text { secs) }}{\text { Improved regardless of protocol } p<0.001} \\
\frac{\text { Hip group baseline } 15+/-5,4 \text { weeks } 17+/-5,8 \text { weeks } 19+/-}{5}\end{array}$ & $\begin{array}{l}\text { Isometric strength } \\
\text { Hip Abductor strength } \\
\text { Hip group increase from } \\
\text { baseline to } 8 \text { weeks } p=0.001 \\
\text { Hip group baseline } 5.2+/-1.5,4 \\
\text { weeks } 6.2+/-1.1,8 \text { weeks } 6.6+/- \\
0.9 \\
\text { Knee group baseline } 5.7+/-2.2,4 \\
\text { weeks } 5.5+/-1.9,8 \text { weeks } 6.2+/- \\
1.8 \\
\text { Hip External Rotator strength } \\
\text { Increased over } 8 \text { weeks both } \\
\text { groups } p=0.004\end{array}$ \\
\hline
\end{tabular}




\begin{tabular}{|c|c|c|c|}
\hline & & $\frac{\text { knee group baseline } 14+/-8,4 \text { weeks } 17+/-7,8 \text { weeks }}{20+/-6}$ & $\begin{array}{l}\text { Hip group baseline } 2.1+/-0.7,4 \\
\text { weeks } 2.5+/-0.7,8 \text { weeks } 2.7+/- \\
0.7 \\
\text { Knee group baseline } 2.1+/-1.0,4 \\
\text { weeks } 2.5+/-0.7,8 \text { weeks } 2.7+/- \\
0.7 \\
\text { Knee Extensor strength } \\
\text { No effect } \\
\text { Hip group baseline } 6.1+/-2.6,4 \\
\text { weeks } 6.8+/-1.9,8 \text { weeks } 7.0+/- \\
1.4 \\
\text { Knee group baseline } 6.3+/-2.1,4 \\
\text { weeks } 6.1+/-1.9,8 \text { weeks } 6.6 \\
+/-1.9\end{array}$ \\
\hline $\begin{array}{l}\text { Ferber et al } \\
2014\end{array}$ & $\begin{array}{l}\text { VAS }(\mathrm{cm})(\text { worst pain in the last week) } \\
\text { Knee group } \\
\text { Baseline } 4.96+/-1.66 \\
\text { At } 6 \text { weeks } 1.99+/-2.05 p<0.05 \\
\text { Difference } 2.98+/-2.08 \\
\text { Hip group } \\
\text { Baseline } 5.12+/-1.66 \\
\text { At } 6 \text { weeks } 1.96+/-1.92 p<0.0 \\
\text { Difference } 3.11+/-2.22\end{array}$ & $\begin{array}{l}\frac{\text { AKPS }}{\text { Knee group }} \\
\text { Baseline } 75.62+/-9.81 \\
\text { At } 6 \text { weeks } 87.67+/-10.53 p<0.05 \\
\text { Difference } 12.90+/-13.55 \\
\text { Hip group } \\
\text { Baseline } 75.00+/-9.74 \\
\text { At } 6 \text { weeks } 87.95+/-11.26 p<0.05 \\
\text { Difference } 12.58+/-11.93\end{array}$ & $\begin{array}{l}\text { MVIC } \\
\text { All results } p<0.05 \text { compared to } \\
\text { baseline } \\
\text { Hip abductor } \\
\text { Knee group } \\
\text { Baseline } 3.15+/-1.19 \\
\text { At } 6 \text { weeks } 3.41+/-1.28 \\
\% \text { change } 8.21 \\
\text { Hip group } \\
\text { Baseline } 3.21+/-1.14 \\
\text { At } 6 \text { weeks } 3.58+/-1.08 \\
\% \text { difference } 11.46 \\
\text { Hip group improved greater } \\
\text { than knee group p<0.05 } \\
\text { Hip extensor } \\
\text { Knee group } \\
\text { Baseline } 2.44+/-1.09 \\
\text { At } 6 \text { weeks } 2.61+/-1.18 \\
\% \text { change } 7.13 \\
\text { Hip group }\end{array}$ \\
\hline
\end{tabular}




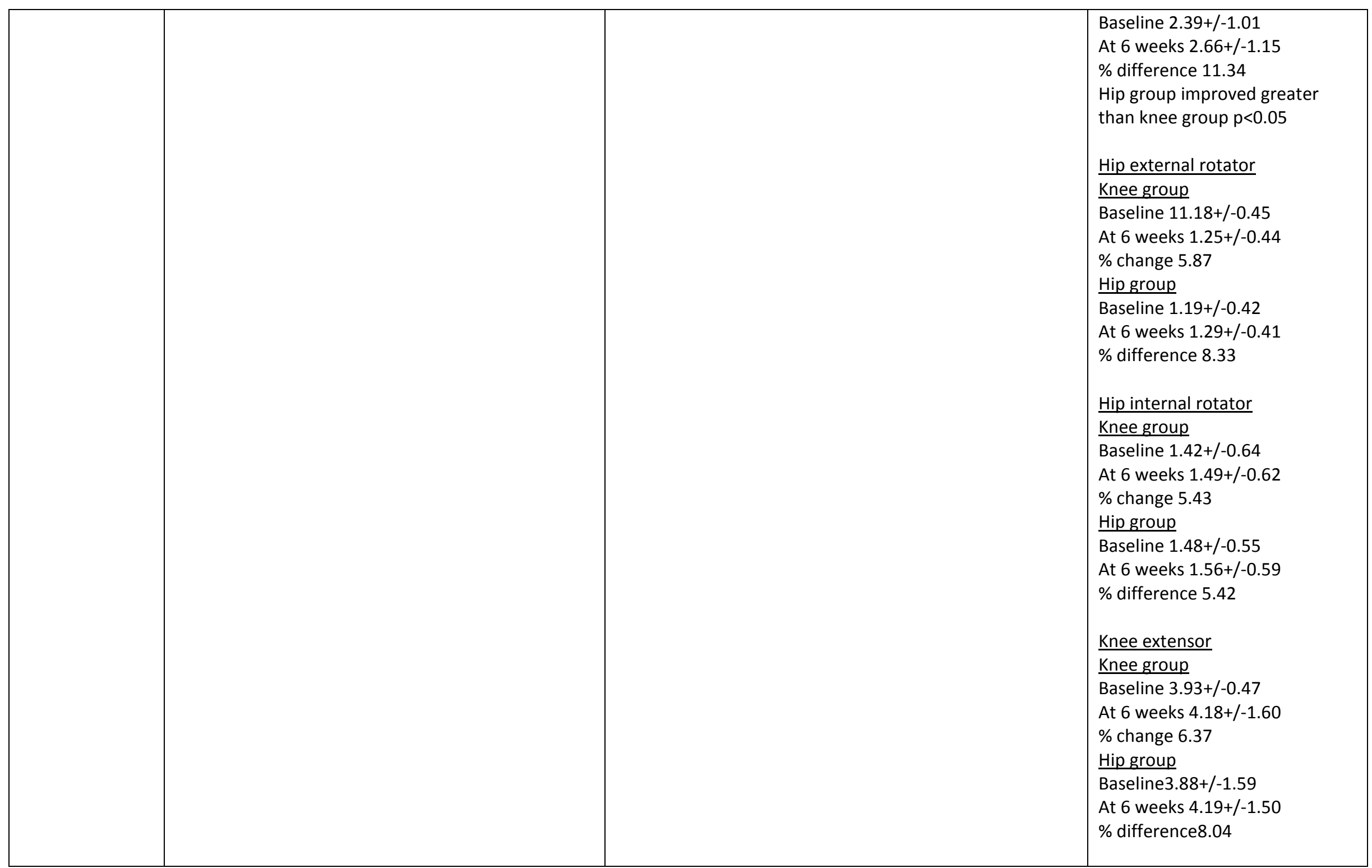




\begin{tabular}{|c|c|c|}
\hline $\begin{array}{l}\text { Fukuda et al } \\
2010\end{array}$ & $\begin{array}{l}\text { NPRS- } 0-10 \\
\text { Ascending stairs } \\
\text { Controls } 4.9+/-2.5 \text { to } 5.0+/-2.5 \\
\text { Knee }-4.9+/-2.9 \text { to } 3.4+/-2.3 \\
\text { Hip }-5.2+/-1.6 \text { to } 3.0+/-1.8 \\
\text { Descending stairs } \\
\text { Controls- } 4.4+/-2.4 \text { to } 4.1+/-2.3 \\
\text { Knee group- } 4.5+/-2.8 \text { to } 3.5+/-2.5 \\
\text { Hip group- } 4.9+/-1.6 \text { to } 2.3+/-1.5 \\
\text { At } 4 \text { weeks only hip group significantly reduction in } \\
\text { pain } p<0.01 \text { compared to baseline } \\
\text { At } 4 \text { weeks knee and hip group significantly lower } \\
\text { than controls, not between groups } p<0.01 \\
\text { Lack of significant difference between knee and hip } \\
\text { groups, both groups improved more than controls } \\
\text { p<0.05 } \\
\text { But hip group showed greater difference for all } \\
\text { scales than knee group }\end{array}$ & $\begin{array}{l}\text { AKPS 0-100 } \\
\text { Controls- } 63.8+/-15.5 \text { to } 64.5+/-11.1 \\
\text { Knee group- } 70.4+/-12.5 \text { to } 80.6+/-13.9 p<0.05 \\
\text { Hip group }-63.9+/-11.7 \text { to } 78.9+/-16.0 p<0.01 \\
\text { Single limb hop (cm) } \\
\text { Controls } 81.0+/-25.5 \text { to } 80.3+/-16.0 \\
\text { Knee group- } 76.1+/-37.7 \text { to } 86.5+/-32 p<0.05 \\
\text { Hip group- } 76.1+/-33.8 \text { to } 91.8+/-34.4 p<0.05 \\
\text { LEFS 0-80 } \\
\text { Controls } 48.8+/-17.0 \text { to } 51.2+/-15.1 \\
\text { Knee group- } 55.6+/-15.9 \text { to } 65.6+/-14.5 p<0.05 \\
\text { Hip group } 49.1+/-11.9 \text { to } 65.7+/-13.5 p<0.05\end{array}$ \\
\hline $\begin{array}{l}\text { Fukuda et al } \\
2012\end{array}$ & $\begin{array}{l}\text { NRPS } 0-10 \\
\text { Ascending stairs } \\
\text { Knee group } 6.6+/-1.2 \text { to } 5.3+/-1.3 \text { ( } 3 \text { months) } 5.5+/- \\
1.2 \text { ( } 6 \text { months) } 6.5+/-1.0 \text { ( } 12 \text { months) } \\
\text { Hip group } 6.2+/-1.1 \text { to } 1.2+/-1.1 \text { ( } 3 \text { months) } 1.7+/- \\
1.0 \text { (6 months) } 2.9+/-0.8 \text { ( } 12 \text { months) } \\
\text { Descending stairs } \\
\text { Knee group 6.4+/- } 1.4 \text { to } 5.0+/-1.2 \text { ( } 3 \text { months) } 5.6+/- \\
1.4 \text { (6 months) } 6.4+/-1.1 \text { ( } 12 \text { months) }\end{array}$ & $\begin{array}{l}\text { LEFS 0-80 } \\
\text { Knee group 49.0+/-13.0 to } 49.4+/-11.2 \text { ( } 3 \text { months) } 47.7+/- \\
10.5 \text { ( } 6 \text { months) } 46.1+/-10.9(12 \text { months) } \\
\text { Hip group } 51.7+/-10.4 \text { to } 74.1+/-5.6 \text { ( } 3 \text { months) } 72.4+/-6.1 \\
\text { ( } 6 \text { months) } 69.6+/-5.2 \text { (12 months) } \\
\text { AKPS } 0-100 \\
\text { Knee group } 61.8+/-9.0 \text { to } 64.6+/-10.2 \text { ( } 3 \text { months) } 62.0+/- \\
9.3 \text { (6 months) } 60.0+/-8.3 \text { ( } 12 \text { months) } \\
\text { Hip group } 65.9+/-8.5 \text { to } 85.7+/-9.0 \text { ( } 3 \text { months) } 81.7+/-7.6 \\
\text { ( } 6 \text { months) } 79.0+/-7.7 \text { (12 months) }\end{array}$ \\
\hline
\end{tabular}




\begin{tabular}{|c|c|c|c|}
\hline & $\begin{array}{l}\text { Hip group } 5.8+/-1.2 \text { to } 1.6+/-1.1 \text { ( } 3 \text { months) } 2.0+/- \\
0.8 \text { ( } 6 \text { months) } 2.5+/-0.9 \text { ( } 12 \text { months) }\end{array}$ & $\begin{array}{l}\text { Single hop test }(\mathrm{cm}) \\
\text { Knee group } 61.7+/-22.6 \text { to } 69.9+/-21.8 \text { ( } 3 \text { months) } 67.3+/- \\
21.5 \text { ( } 6 \text { months) } 65.6+/-21.2(12 \text { months) } \\
\text { Hip group } 69.9+/-10.4 \text { to } 85.7+/-10.2 \text { ( } 3 \text { months) } 84.0+/- \\
10.9 \text { ( } 6 \text { months) } 82.3+/-10.2 \text { ( } 12 \text { months) }\end{array}$ & \\
\hline Ismail et al 2013 & $\begin{array}{l}\text { VAS (cm) (average pain in last week) } \\
\text { CKC Group 4.5+/-1.8 to } 2.3+/-1.1 \\
\text { Difference } 2.26+/-1.3 \\
\text { CKC+OKC Group 5.3+/-1.6 to } 2.0+/-1.1 \\
\text { Difference } 3.2+/-0.9\end{array}$ & $\begin{array}{l}\text { AKPS 0-100 } \\
\text { CKC Group } 76.4+/-10.4 \text { to } 85.0+/-6.7 \\
\text { Difference } 8.6+/-7.3 \\
\text { CKC+OKC Group } 71.5+/-7.8 \text { to } 85.1+/-6.2 \\
\text { Difference } 13.7+/-5.5\end{array}$ & $\begin{array}{l}\text { Isokinetic strength } \\
\text { Both groups improved from } \\
\text { baseline } p<0.05 \\
\text { Hip abductor concentric } \\
\text { CKC group } 2.1+/-0.6 \text { to } 2.5+/-0.7 \\
\text { (6 weeks) } \\
\text { CKC+OKC group } 1.7+/-0.6 \text { to } \\
2.4+/-0.8 \text { ( } 6 \text { weeks) } \\
\text { Hip abductor eccentric } \\
\text { CKC group } 2.2+/-0.5 \text { to } 2.4+/-0.5 \\
\text { CKC+OKC group } 2.0+/-0.6 \text { to } \\
2.4+/-0.8 \text { ( } 6 \text { weeks) } \\
\text { Hip external rotator concentric } \\
\text { CKC group } 1.0+/-0.4 \text { to } 1.2+/-0.4 \\
\text { CKC+OKC group } 0.9+/-0.6 \text { to } \\
1.3+/-0.6 \\
\text { Hip external rotator eccentric } \\
\text { CKC group } 1.4+/-0.4 \text { to } 1.6+/-0.3 \\
\text { (6 weeks) } \\
\text { CKC+OKC group 1.4+/- } 0.4 \text { to } \\
1.8+/-0.8\end{array}$ \\
\hline $\begin{array}{l}\text { Khayambashi et } \\
\text { al } 2012\end{array}$ & $\begin{array}{l}\text { VAS }(\mathrm{cm}) \text { (average pain of both knees while } \\
\text { performing activities that aggravated symptoms } \\
\text { during the previous week) } \\
\text { Exercise group } 7.9+/-1.7 \text { to } 1.4+/-1.9 \text { at } 8 \text { weeks } \\
\text { p }<0.001 \\
\text { remained reduced at } 6 \text { months }(1.7+/-2.7) \\
\text { control group } 6.6+/-2.0 \text { to } 6.7+/-2.4\end{array}$ & $\begin{array}{l}\text { WOMAC } 0-96, \text { larger numbers indicating worse health } \\
\text { status } \\
\text { Exercise group } 54.0+/-18.1 \text { to } 10.7+/-16.1 p<0.001 \\
\text { Control group } 55.9+/-15.7 \text { to } 59.9+/-12.6\end{array}$ & $\begin{array}{l}\text { Isometric strength } \\
\text { Hip Abductor strength } \\
\text { Hip group Right side } 11.6+/-2.3 \\
\text { to } 15.3+/-2.5 \text { (8 weeks) } p<0.001 \\
\text { Left side- } 11.2+/-2.7 \text { to } 15.9+/- \\
3.1(8 \text { weeks)p }<0.001 \\
\text { Control group Right side } 12.3+/- \\
2.9 \text { to } 11.2+/-2.5\end{array}$ \\
\hline
\end{tabular}




\begin{tabular}{|c|c|c|}
\hline & & $\begin{array}{l}\text { Left side }-12.5+/-3.7 \text { to } 11.4+/- \\
3.1 \text { ( } 8 \text { weeks) } \\
\text { Hip External Rotator strength } \\
\text { Hip group } \\
\text { Right side } 8.6+/-2.3 \text { to } 11.8+/- \\
2.2(8 \text { weeks)p<0.001 } \\
\text { Left side }-7.0+/-1.8 \text { to } 10.9+/- \\
2.6(8 \text { weeks)p<0.001 } \\
\text { Control group } \\
\text { R side } 8.9+/-2.1 \text { to } 8.3+/-2.3 \\
\text { (8weeks) } \\
\text { Left side } 7.5+/-1.6 \text { to } 7.3+/-1.9 \\
\text { (8weeks) }\end{array}$ \\
\hline $\begin{array}{l}\text { Nakagawa et al } \\
2008\end{array}$ & $\begin{array}{l}\frac{V A S(\mathrm{~cm})}{\text { Controls }} \\
\text { Usual pain } 4.7+/-2.6 \text { to } 4.0+/-2.6 p=0.31 \\
\text { Worst pain } 5.5+/-1.5 \text { to } 3.4+/-1.9 p=0.2 \\
\text { Stair climbing } 5.0+/-3.4 \text { to } 2.6+/-2.8 p=0.13 \\
\text { Descending stairs } 4.7+/-3.3 \text { to } 2.0+/-2.4 p=0.43 \\
\text { Squatting } 4.8+/-3.0 \text { to } 3.0+/-3.1 p=0.12 \\
\text { Prolonged sitting } 5.2+/-2.8 \text { to } 2.9+/-3.1 p=0.09 \\
\text { Intervention group- } \\
\text { Usual pain } 3.8+/-2.1 \text { to } 1.1+/-1.2 p=0.03 \\
\text { Worst pain } 5.0+/-2.1 \text { to } 1.4+/-1.3 p=0.03 \\
\text { Stair climbing } 3.5+/-3.7 \text { to } 0.4+/-0.6 p=0.04 \\
\text { Descending stairs } 4.5+/-3.1 \text { to } 0.3+/-0.4 p=0.03 \\
\text { Squatting } 5.7+/-3.2 \text { to } 0.4+/-0.6 p=0.02 \\
\text { Prolonged sitting } 2.0+/-3.2 \text { to } 1.1+/-1.6 p=0.14\end{array}$ & 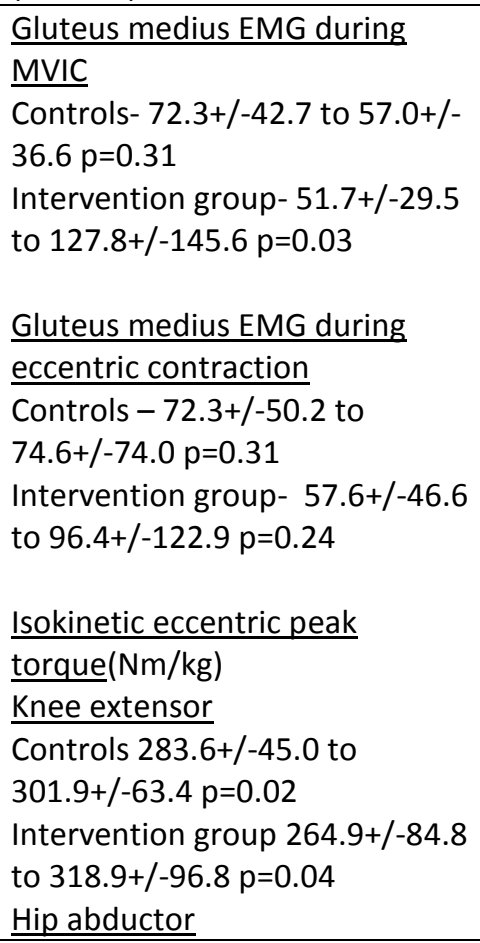 \\
\hline
\end{tabular}




\begin{tabular}{|c|c|c|c|}
\hline & & & $\begin{array}{l}\text { Controls } 114.6+/-32.1 \text { to } \\
120.4+/-30.4 \\
\text { Intervention group } 89.1+/-29.5 \\
\text { to } 102.2+/-19.8 \\
\text { Hip lateral rotator } \\
\text { Controls } 60.4+/-16.5 \text { to } 62.9+/- \\
24.9 \\
\text { Intervention group } 55.5+/-14.6 \\
\text { to } 59.4+/-18.9\end{array}$ \\
\hline $\begin{array}{l}\text { Razeghi et al } \\
2010\end{array}$ & $\begin{array}{l}\text { VAS(no detail recorded) } \\
\text { Hip and knee group } 6.68+/-1.62 \text { to } 3.37+/-1.5 \\
p=0.001 \\
\text { Knee group } 6.31+/-1.25 \text { to } 4.81+/-1.79 p=0.005\end{array}$ & & $\begin{array}{l}\text { Isometric muscle strength of hip } \\
\text { flexors, abductors, external } \\
\text { rotators, internal rotators, } \\
\text { adductors and extensors } \\
\text { measured. } \\
\text { No numerical data recorded for } \\
\text { specific exercise groups. Pooled } \\
\text { data recorded for successful } \\
\text { versus unsuccessful treatment } \\
\text { (defined as } 1.5 \mathrm{~cm} \text { reduction in } \\
\text { VAS) irrespective of group. }\end{array}$ \\
\hline Song et al 2009 & $\begin{array}{l}\text { VAS }(\mathrm{cm})(\text { worst pain previous week) } \\
\text { Leg press group- } 4.85+/-2.49 \text { to } 2.26+/-2.2 p<0.005 \\
\text { Leg press plus hip adduction group } 4.8+/-2.26 \text { to } \\
2.62+/-2.51 p<0.005 \\
\text { Controls } 4.99+/-2.18 \text { to } 4.81+/-2.55 p=0.715\end{array}$ & $\begin{array}{l}\text { Lysholm scale } \\
\text { Leg press group } 75.7+/-12.8 \text { to } 86.5+/-10.4 p<0.005 \\
\text { Leg press plus hip adduction group } 74.8+/-12.1 \text { to } 85.7+/- \\
8.5 p<0.005 \\
\text { Controls } 75.1+/-9.3 \text { to } 75.7+/-10.9 p=0.714\end{array}$ & $\begin{array}{l}\text { VMO cross-sectional area }\left(\mathrm{cm}^{2}\right) \\
\text { Leg press group } 3.75+/-1.59 \text { to } \\
4.46+/-1.90 \mathrm{p}<0.005 \\
\text { Leg press plus hip adduction } \\
\text { group } 3.67+/-1.45 \text { to } 4.24+/- \\
1.43 p=0.004 \\
\text { Controls } 3.39+/-1.47 \text { to } 3.38+/- \\
1.52 p=0.962 \\
\text { VMO volume }\left(\mathrm{cm}^{3}\right) \\
\text { Leg press group } 3.38+/-2.37 \text { to } \\
4.45+/-2.52 p<0.005 \\
\text { Leg press plus hip adduction } \\
3.04+/-2.18 \text { to } 4.12+/-1.83 \\
p<0.005 \\
\text { Controls } 2.76+/-2.01 \text { to } 2.82+/- \\
1.91 p=0.838\end{array}$ \\
\hline
\end{tabular}




\begin{tabular}{|c|c|c|c|}
\hline & & & \\
\hline Boling et al 2006 & $\begin{array}{l}\text { VAS }(\text { no detail recorded) } \\
\text { no values recorded } \\
\text { PFP group reduced pain } p=0.001 \text {, control group no } \\
\text { change, significant by group interaction } p=0.001\end{array}$ & $\begin{array}{l}\frac{F I Q}{N o} \text { values recorded } \\
\text { Increased for PFP group from baseline, control group ISQ, } \\
\text { group interaction effect } p=0.001\end{array}$ & $\begin{array}{l}\frac{\text { Gluteus medius onset ascending }}{\text { stairs }} \\
\text { PFP group -81.64+/-153.33 to - } \\
\text { 49.56+/-136.7 } \\
\text { Controls }-19.54+/-52.21 \text { to - } \\
32.38+/-41.34 \\
\text { Gluteus medius onset } \\
\text { descending stairs } \\
\text { PFP group -158.93+/-69.30 to - } \\
\text { 133.76+/-96.17 } \\
\text { Controls }-154.26+/-58.70 \text { to - } \\
\text { 131.95+/-59.94 } \\
\text { Gluteus medius duration } \\
\text { ascending stairs } \\
\text { PFP group } 631.67+/-74.03 \text { to } \\
\text { 578.48+/-148.17 } \\
\text { Controls } 621.77+/-152.34 \text { to } \\
\text { 606.64+/-154.35 } \\
\text { Gluteus medius duration } \\
\text { descending stairs } \\
\text { PFP group } 329.64+/-85.85 \text { to } \\
\text { 303.24+/-125.31 } \\
\text { Controls 363.43+/-145.72 to } \\
\text { 357.46+/-165.35 } \\
\text { Onset- significantly earlier } \\
\text { during descent compared with } \\
\text { ascent, duration in concentric } \\
\text { phase longer than eccentric } \\
\text { Pre and post test values } \\
\text { onset/duration no significant }\end{array}$ \\
\hline
\end{tabular}




\begin{tabular}{|c|c|c|c|}
\hline & & & $\begin{array}{l}\text { difference and no significant } \\
\text { difference between groups }\end{array}$ \\
\hline $\begin{array}{l}\text { Earl and Hoch } \\
2011\end{array}$ & $\begin{array}{l}\frac{\text { VAS }(\mathrm{mm})(\text { usual pain in a day) }}{40+/-18 \text { to } 5+/-7 \mathrm{p}<0.0005,15 \text { improved }>20 \mathrm{~mm}}\end{array}$ & $\frac{\text { AKPS }}{70.4+/-11.2 \text { to } 83.7+/-11.2 p=0.001,2 \text { unsuccessful }}$ & \\
\hline $\begin{array}{l}\text { Ferber et al } \\
2011\end{array}$ & $\begin{array}{l}\text { VAS }(\mathrm{cm}) \text { (average amount of pain during past week } \\
\text { when running) } \\
5.8+/-2.10 \text { to } 3.30+/-1.90 \\
43.1 \% \text { reduction in score } p=0.01\end{array}$ & & 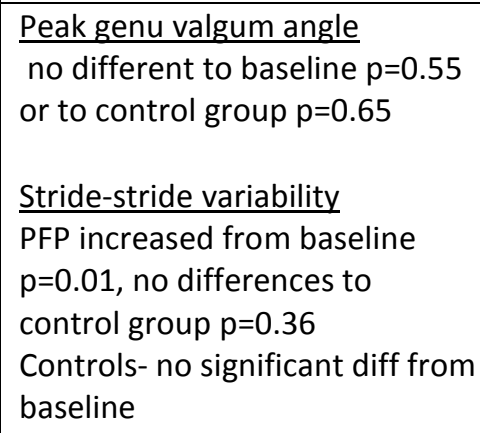 \\
\hline $\begin{array}{l}\text { Khayambashi et } \\
\text { al } 2014\end{array}$ & $\begin{array}{l}\text { VAS(cm) ("based on activities that aggravate pain in } \\
\text { the last week") } \\
\text { Hip group } \\
7.63+/-1.79 \text { to } 2.11+/-1.6 \text { ( } 8 \text { weeks) to } 2.00+/-1.97 \text { (6 } \\
\text { months) } \\
\text { Knee group } \\
6.91+/-1.94 \text { to } 3.27+/-2.19 \text { (8 weeks) to } 4.00+/- \\
2.44 \text { ( } 6 \text { months) }\end{array}$ & 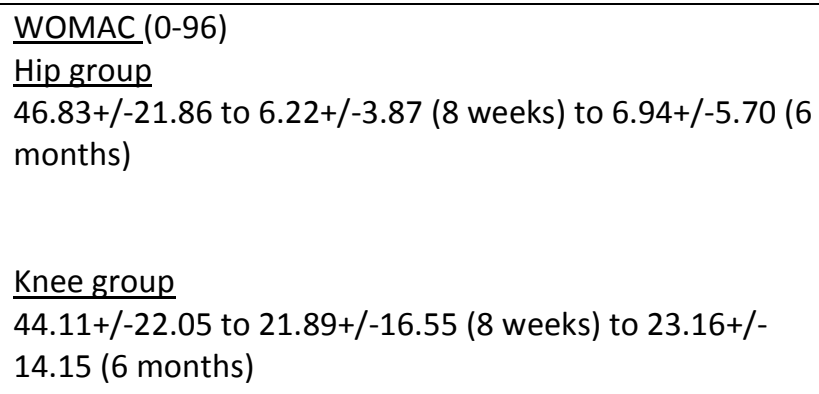 & \\
\hline Lowry 2008 & $\begin{array}{l}\text { VAS(no definition) } \\
\text { Pt } 2-7 / 10 \text {-to } 3 / 10 \text { at } D / C 2 / 10 \text { ( } 6 \text { months) } \\
\text { Pt } 3-6 / 10 \text {-to } 1 / 10 \text { at } D / C \text { to } 0 / 10 \text { (6 months) } \\
\text { Pt } 4-2 / 10 \text {-to } 0 / 10 \text { at } D / C \text { to } 2 / 10 \text { (6 months) } \\
\text { Pt } 5-8 / 10 \text {-to } 3 / 10 \text { at } D / C \text { to } 0 / 10 \text { (6 months) }\end{array}$ & 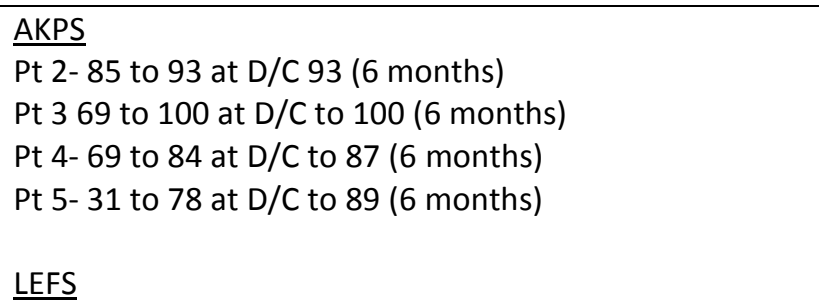 & \\
\hline
\end{tabular}




\begin{tabular}{|c|c|c|c|}
\hline & & $\begin{array}{l}\text { Pt } 2-71 \text { to } 75(\mathrm{D} / \mathrm{C}) \text { to } 72 \text { (6 months) } \\
\text { Pt } 3-59 \text { to } 77(\mathrm{D} / \mathrm{C}) \text { to } 80 \text { (6 months) } \\
\text { Pt } 4-58 \text { to } 68(\mathrm{D} / \mathrm{C}) \text { to } 76 \text { (6 months) } \\
\text { Pt } 528 \text { to } 61(\mathrm{D} / \mathrm{C}) \text { to } 69 \text { (6 months) } \\
\frac{\mathrm{GRC}}{\text { Pt } 2-0 \text { (4th visit) to } 0 \text { (6 months) }} \\
\text { Pt } 3-6 \text { (4th visit) to } 7 \text { (6 months) } \\
\text { Pt } 44 \text { (4th visit) to } 3 \text { (6 months) } \\
\text { Pt } 56 \text { (4th visit) to } 7 \text { (6 months) }\end{array}$ & \\
\hline Mascal 2003 & $\begin{array}{l}\text { VAS (greatest amount of pain during most pain } \\
\text { provoking activity) } \\
\text { improved from } 10 / 10 \text { to } 2 / 10\end{array}$ & AKPS improved from 70 to 84 & No kinematic data \\
\hline Tyler 2006 & $\begin{array}{l}\text { VAS }(\mathrm{cm}) \text { (with ADL) } \\
\text { With exercise } 5.8+/-0.4 \text { to } 3.0+/-0.4 p<0.001 \\
\text { Treatment success defined minimum } 1.5 \mathrm{~cm} \\
\text { reduction- } \\
26 \text { knees ( } 21 \mathrm{pts}) \text { successful, } 17 \text { knees (14pts) } \\
\text { unsuccessful }\end{array}$ & & \\
\hline
\end{tabular}

VAS-visual analogue scale

AKPS- anterior knee pain scale

LEFS- lower extremity functional scale

NRPS- numerical rating pain scale

GRC -Global rating of change

WOMAC- Western Ontario and McMaster Universities questionnaire

ADL- activities of daily living

\section{pt- patient}

OKC- open kinetic chain

PFP- patellofemoral pain
FST- functional stabilisation training

ST- standard training

CKC- closed kinetic chain 
$\underline{\text { Neuromuscular exercise }}$

Two case series including 20 female runners with both unilateral and bilateral PFP, aged 18-45 years investigated neuromuscular exercise (Tables 10-11). One paper analysed the dominant leg in subjects with bilateral symptoms, the other the limb with more marked hip adduction when running. 
Table 10 Study characteristics of neuromuscular control exercise

\begin{tabular}{|c|c|c|c|c|c|c|c|c|}
\hline Study & Study design & Population & Sample size & $\begin{array}{l}\text { Sample } \\
\text { size } \\
\text { calculation }\end{array}$ & Age & Gender & Inclusion & Unilateral/bilateral PFP \\
\hline $\begin{array}{l}\text { Noehren et } \\
\text { al } 2011\end{array}$ & Case series & Runners & 10 & Yes & $\begin{array}{l}23.3 \\
\text { years } \\
(5.8) \\
\text { Range } \\
\text { not } \\
\text { recorded }\end{array}$ & female & $\begin{array}{l}\text { Duration } \\
>2 / 12 \\
\text { VAS } \\
>4 / 10\end{array}$ & $\begin{array}{l}\text { Unilateral/bilateral } \\
\text { If bilateral PFP leg with greater } \\
\text { hip adduction used for analysis }\end{array}$ \\
\hline $\begin{array}{l}\text { Willy \& } \\
\text { Davis } 2012\end{array}$ & Case series & Runners & 10 & Yes & $\begin{array}{l}18-40 \\
\text { years }\end{array}$ & female & $\begin{array}{l}\text { VAS } \\
>3 / 10 \\
\text { when } \\
\text { running }\end{array}$ & $\begin{array}{l}\text { Unilateral/bilateral If bilateral } \\
\text { most dominant limb used for } \\
\text { analysis }\end{array}$ \\
\hline $\begin{array}{l}\text { Willy \& } \\
\text { Davis } 2013\end{array}$ & $\begin{array}{l}\text { Case study of } 2 \text { runners } \\
\text { from paper above, } \\
\text { additional data }\end{array}$ & Runners & 2 & N/A & $\begin{array}{l}18- \\
40 y e a r s\end{array}$ & female & & $\begin{array}{l}\text { One unilateral, one bilateral- } \\
\text { most painful knee assessed }\end{array}$ \\
\hline
\end{tabular}

PFP- patellofemoral pain

VAS- visual analogue scale 
Table 11 Exercise protocols: Neuromuscular exercise

\begin{tabular}{|c|c|c|c|c|c|}
\hline Study & Exercise & Progression & $\begin{array}{l}\text { Time frame } \\
\text { for exercise }\end{array}$ & $\begin{array}{l}\text { Frequency of } \\
\text { exercise }\end{array}$ & $\begin{array}{l}\text { Follow up } \\
\text { period }\end{array}$ \\
\hline Noehren et al 2011 & $\begin{array}{l}\text { Real time kinematic visual feedback of hip } \\
\text { adduction during stance phase in } 30 \text { minute run }\end{array}$ & $\begin{array}{l}\text { Increase in running time } \\
\text { from } 15 \text { to } 30 \text { minutes. } \\
\text { Reduction in visual } \\
\text { feedback from continual } \\
\text { over last } 4 \text { sessions }\end{array}$ & 2 weeks & $4 \times$ week & 1 month \\
\hline $\begin{array}{l}\text { Willy, Scholz \& Davis } \\
2012\end{array}$ & $\begin{array}{l}\text { Real time mirror visual feedback of hip adduction } \\
\text { and internal rotation during stance phase in } 30 \\
\text { minute run }\end{array}$ & $\begin{array}{l}\text { Increase in running time, } \\
\text { from } 15 \text { to } 24 \text { minutes } \\
\text { session 1-4, } 30 \text { minutes } \\
\text { run final session. } \\
\text { Reduction in visual } \\
\text { feedback over last } 4 \\
\text { sessions from full } \\
\text { running time to } 3 \\
\text { minutes }\end{array}$ & 2 weeks & 4xweek & 3 months \\
\hline Willy \& Davis 2013 & $\begin{array}{l}\text { Real time mirror visual feedback of hip adduction } \\
\text { and internal rotation during stance phase in } 30 \\
\text { minute run }\end{array}$ & $\begin{array}{l}\text { Increase in running time, } \\
15 \text { minutes to } 24 \\
\text { minutes in sessions } 1-4, \\
30 \text { minutes run final } \\
\text { session. } \\
\text { Reduction in visual } \\
\text { feedback over last } 4 \\
\text { sessions, last visit } 3 \\
\text { minutes feedback. }\end{array}$ & 2 weeks & $4 \times$ week & 3 months \\
\hline
\end{tabular}


Hip neuromuscular training was effective for reducing pain, improving function and improving hip kinematics (Table 12). Pain reduced considerably, being in the region of $90 \%$ reduction in both studies, which was maintained at follow-up despite a trend for the hip mechanics to revert toward baseline.

A significant reduction in hip adduction and contra-lateral pelvic drop was evident in both studies and reduction of $23 \%$ in hip internal rotation in one (Noehren et al., 2011); this did not reach significance. There were kinematic improvements in untrained activities, in single leg squat and stepdescent, demonstrating transfer of motor skill learning. 
Table 12 Outcomes with neuromuscular exercise

\begin{tabular}{|c|c|c|c|}
\hline Study & Pain & Functional outcome & Kinematics \\
\hline $\begin{array}{l}\text { Willy, Scholz \& Davis } \\
2013\end{array}$ & $\begin{array}{l}\text { Reduction in pain ( no data on figures) } p>0.05 \\
\text { effect size } 3.81-7.61\end{array}$ & 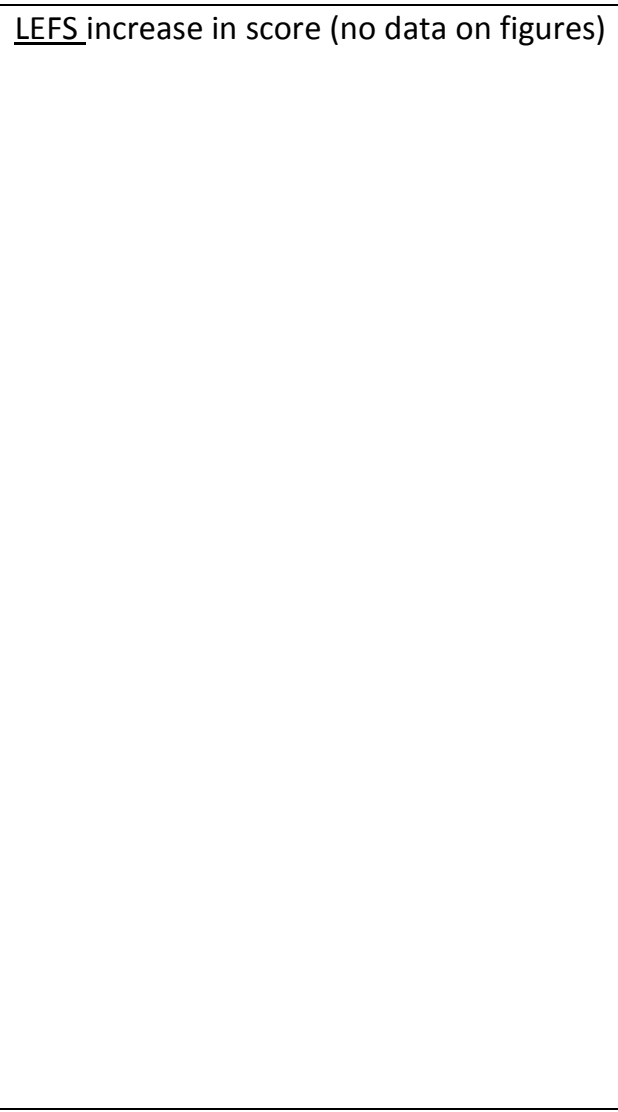 & $\begin{array}{l}\text { Running: } \\
\text { Reduction in peak HADD } 20.7(1.0) \text { to } \\
14.8(3.1) p=0.02 \text {, increased to } 15.9 \text { (2.7) at } 1 \\
\text { month16.4(2.5) at } 3 \text { months } \\
\text { Reduction in peak CPD }-9(2.5) \text { to }-7.5(2.2) \\
\text { post to }-7.5(2.3) 1 \text { month to }-7.0(2.2) \text { at } 3 \\
\text { months } \\
\text { Peak Thigh ADD } 9.8(1.2) \text { to } 7.2(2.7) \text { post to } \\
7.3(1.8) 1 \text { month to } 8.1(1.4) \text { at } 3 \text { months } \\
\text { HABDM }-1.180(0.185) \text { to }-1.054(0.184) \text { post } \\
\text { to }-1.074(0.173) 1 \text { month to }-1.153(0.145) 3 \\
\text { months } \\
\text { HIR } 8.6(5.4) \text { to } 7.1(8.7) \text { to } 6.2(7.9) \text { to } 5.7(6.3) \\
3 \text { months } \\
\text { Squat } \\
\text { Peak HADD } 11.6(3.4) \text { to } 7.6(2.6) \text { post to } \\
\text { Peak CPD } 0.6(2.0) \text { to } 2.3(2.5) \text { months } 2.6(2.6) 1 \\
\text { month to } 2.2(2.4) 3 \text { months } \\
\text { Peak thigh ADD } 11.5(2.0) \text { to } 10.1(2.2) \text { to } \\
9.8(2.2) \text { to } 10.1(1.9) \\
\text { HABDM }-0.470(0.064) \text { to }-0.412(0.070) \text { to }- \\
0.431(0.071) \text { to }-0.477(0.039) \\
\text { HIR } 3.0(6.4) \text { to } 5.9(8.5) \text { to } 3.9(7.6) \text { to } 4.0(6.4) \\
\text { Step descent }\end{array}$ \\
\hline
\end{tabular}




\begin{tabular}{|c|c|c|c|}
\hline & & & 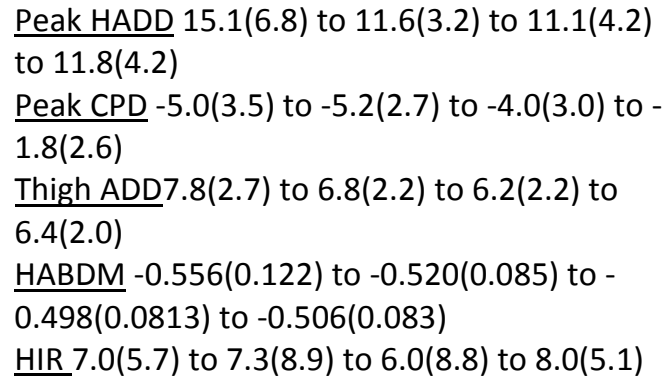 \\
\hline Willy \& Davis 2013 & $\begin{array}{l}\text { Runner } 1 \text { VAS running pre } 4 / 10 \text { to post } 0.5 / 10 \\
1 / 122.5 / 103 / 120 / 10 \\
\text { step ascent pre } 2 / 10 \text { to post } 0 / 101 / 120 / 103 / 12 \\
0 / 10 \\
\text { Runner } 2 \text { pre } 3.5 / 10 \text { to post } 0 / 10,0 / 101 / 12,0 / 10\end{array}$ & $\begin{array}{l}\text { Runner } 1 \text { LEFS post } 75 / 80,1 \text { month } 79 / 80 \\
\text { Runner } 2 \text { LEFS } 80 / 80 \text { at post, } 1 \text { months, } 3 \\
\text { months }\end{array}$ & $\begin{array}{l}\frac{\text { Running }}{\text { Runner } 1} \\
\frac{\text { HADD } 20.8 \text { to } 15.6}{\text { CPD }-6.4 \text { to }-3.8} \\
\text { HIR }-1.1 \text { to } 1.5 \\
\frac{\text { Runner } 2}{\text { HADD } 22.5 \text { to } 16.3} \\
\text { CPD }-8.9 \text { to }-7.2 \\
\text { HIR } 7.7 \text { to } 6.2 \\
\frac{\text { Step ascent }}{\text { Runner } 1} \\
\text { HADD } 16.9 \text { to } 13.9 \\
\text { CPD }-10.5 \text { to }-6.9 \\
\text { HIR }-9.9 \text { to }-5.4 \\
\frac{\text { Runner } 2}{\text { HADD } 14.1 \text { to } 14.6} \\
\text { CPD }-12.6 \text { to }-14.1 \\
\text { HIR }-1.8 \text { to } 1.4\end{array}$ \\
\hline
\end{tabular}

\section{HADD- hip adduction}

HIR- hip internal rotation
SLS- single leg stand

ADD- adduction
HABDM-Internal hip abduction moment

CPD- contralateral pelvic drop
VAS- visual analogue scale

LEFS- lower extremity functional scale 


\section{DISCUSSION}

This review aimed to investigate the outcome of hip exercise in PFP. There was strong evidence for the benefit of hip strengthening exercise and limited evidence for the benefit of neuromuscular exercise. A strength of this review was consistent support across all types of studies.

Although RCTs are considered to be the gold standard of research they can be equally or more flawed than other study designs (Grossman and Mackenzie,2005). Paradoxically, as the features that ensure the robustness of internal validity are controlled there have been questions raised about the integration of the findings from RCTs into clinical practice (Koes and Hoving 1998; Milanese, 2011). The support from observational studies with lower rigour but with higher generalisability provides a sound rationale for the use of proximal exercise in clinical practice.

\section{Hip strengthening exercise}

Hip strengthening was found to be an effective form of exercise for people with PFP. However, improvements in comparative exercise protocols were also demonstrated. There was a tendency for superior results with the hip programmes, although this was not consistent. The lack of difference in the exercise regimes may relate to choice of exercise in both the trial and control groups, confounding factors being poorly addressed and inadequate rehabilitation protocols.

The majority of studies included weight bearing exercise with eleven including exercises with high or very high activation of gluteus medius (Table 8). There was less focus on gluteus maximus, with four studies including exercises that have demonstrated high level activation. However, it was common for control exercise groups to include exercises that activated gluteal muscles to a high level; this occurred in nine studies including all three studies that compared knee and hip protocols.

Only one study solely compared OKC hip and OKC knee exercise (Dolak et al., 2011). There was a $43 \%$ reduction in pain in the hip group compared to less than $3 \%$ for the knee group after a four 
week programme which was followed by functional exercises for both groups. The second phase was predominantly exercises in single leg stance. There was no between group difference at the end of the programme, but there were more pronounced differences in the hip group with all outcome measures. It could be argued that this was mainly targeted towards proximal strengthening, supported by the fact that both groups showed improvement in hip external rotator but not quadriceps strength.

The addition of OKC hip exercise to an exercise programme resulted in superior improvements in pain and function that were clinically relevant. However, all included OKC external rotation exercises at 90 degrees flexion. This may not have been the optimum position to strengthen this muscle group due to the reverse rotary action of some of the external rotators at 90 degrees flexion (Neumann, 2010). Outcomes may be further improved with OKC rotator exercise nearer to zero degrees or with exercises reflecting the muscles' main functional role, which is controlling pelvic and trunk rotation in single leg stance(Neumann, 2010); this was included in only three studies in this review .

Exercises targeting the abductors and external rotators predominated. Although there is evidence for hip abductor and external rotator weakness in PFP, hip strength deficits are not exclusive to these muscle groups (Rathleff et al., 2014). It is possible that the focus on hip abductor and external rotator exercise is too simplistic.

This review failed to demonstrate that hip adduction exercise was beneficial. However, this was based on one study investigating one isometric exercise. There may be an argument for incorporating adductor strengthening as there is evidence to show a deficit in isometric adduction strength in PFP (Rathleff et al., 2014). Additionally, the adductors contribute to the eccentric control of hip internal rotation, are considered to be important flexors and extensors of the hip, have a bidirectional sagittal plane torque which is useful for powering cyclic activities including sprinting 
and raising and descending a deep squat, and contribute to pelvis stability (Charnock et al., 2009; Gottschall et al., 2012; Leighton, 2006; Neumann, 2010).

Rehabilitation protocols were variable, with little adherence to recommended guidelines for exercise (ACSM's Guidelines for Exercise Testing and Prescription, 2010); future research protocols should take this into account.

Nine trials had mixed gender groups, including two out of the three studies investigating knee versus hip protocols, which may confound the outcome as a recent study demonstrated knee extensor deficits but no hip weakness in males with PFP (Bolgla et al., 2014). Additionally, it has been shown that there are gender specific differences in hip abduction strength (Brent et al.,2013; Ramskov et al., 2014), gluteal activation (Nakagawa et al., 2012) and trunk and lower limb kinematics (Nakagawa et al., 2012, Willy et al., 2012a) in both the normal and PFP population. There have been shown to be differences in hip strength deficits in males and females in PFP (Rathleff et al., 2014) suggesting that rehabilitation and future research trials should be gender specific.

Seven studies did not take activity levels into account. Strength gains in untrained participants improve faster than trained (Kraemer and Rataness, 2004) which may confound results.

Differences in the presentation of hip strength in individuals with unilateral and bilateral PFP have been demonstrated, although this is limited. (Cichanowski et al., 2007; Maghaeles et al., 2010). Further research is needed to establish whether this data is substantiated; if this is the case it may be preferable to sub-group accordingly in future research. A consensus from PFP researchers for standardisation of methodologywith subjects with bilateral symptoms is recommended to enable meaningful comparison between trials.

Evidence for alteration in lower limb kinematics with proximal strengthening was conflicting. Two out of three studies showed an improvement. However, both included verbal instruction on lower limb alignment, suggesting that the alteration may not relate to strength gains. 


\section{Neuromuscular exercise}

Motor retraining was found to result in rapid and lasting reduction in PFP symptoms. A considerable reduction in pain occurred over a short time-frame in both neuromuscular studies given that participants had symptoms for an average of 75 and 51 months respectively. Although the effects were very positive the participantswere a pre-selected group who demonstrated abnormal hip alignment during running, which in one study was 10 out of 85 subjects analysed (Noehren et al., 2011). There may be a subset of people with PFP who would benefit from this type of rehabilitation. The subjects were all runners, and were altering kinematics in running. The results may be different in different PFP populations as motor skill acquisition is highly task specific and dependent on age, prior skill and activity levels (Dayan and Cohen, 2011).

The two main studies did not include strength measures or record EMG activity. Two subjects from the cohort of one study had EMG and strength assessments but did not show consistent results (Willy and Davis, 2013). It is therefore unknown whether the alteration in kinematics facilitated strength gain, improved activation patterns in the gluteal muscles, or whether the results relate to cortical neuroplastic changes.

The improvements in motor skills were transferred to the untrained tasks of step down (Willy et al., 2012b) and single leg squat (Noehren et al., 2011; Willy et al., 2012b) with significant improvement in hip adduction, which was the focus of the motor retraining task. This suggests that similar motor sequences may offer the greatest potential for positive transfer.

Longer term follow up demonstrated a trend for the hip mechanics to revert toward baseline values. The authors suggested this was due to subject's initial over-correction of the abnormal mechanics (Willy et al., 2012b). However, it may be the case that the re-training had not been sufficient to consolidate the altered mechanics. New motor patterns are incorporated into existing neural circuits and this change may be impeded by old motor behaviours (Adkins et al., 1985). Long term retention 
of motor skill acquisition is strongly dependent on successful consolidation (Dayan and Cohen, 2011), which requires repetition (Luft and Buitrago, 2005), and is also dependent on the extent of conscious attention and skill (Hodges, 2011; Remple et al., 2001). Retraining running patterns in a group of runners may take more intense feedback over a longer timeframe.

\section{Limitations}

The results of this review should be interpreted with caution as it was commonly found that studies were under-powered due to low numbers. It was not possible to do a meta-analysis due to widespread methodological heterogeneity.

\section{CONCLUSION}

The evidence consistently demonstrated that both hip strengthening and neuromuscular exercise has a beneficial effect on pain and function in PFP subjects. Motor skill retraining was found to be effective in a participant group demonstrating abnormal kinematics. As PFP is multi-factorial in nature it may be preferential to assess hip strength deficits prior to instigating strengthening trials.

There is a bias towards exercises addressing abductor and external rotator strengthening; future research should consider identifying muscle group weakness prior to strengthening trials.

Hip and knee strengthening programmes were shown to be equally effective. However, results show promising results for the addition of hip exercise to a knee programme. Both OKC and CKC hip exercises were shown to be effective in the management of PFP.

A consensus from PFP researchers for standardisation of methodology is recommended to enable meaningful comparison between trials. 
The relationships between muscle strength, endurance, neuromuscular control and kinematics need to be established with further research in order for a better understanding of proximal hip factors and PFP. 


\section{REFERENCES}

ACSM's Guidelines for Exercise Testing and Prescription. (2010). (Eighth ed.). Philadelphia: Wolters Kluwer/ Lippincott Williams \& WIlkins.

Adkins, D.L., Boychuk, J., Remple, M.S., Kleim, J.A. Motor training induces experience specific patterns across motor cortex and spinal cord. Journal of Applied Physiology 2005; 101(6): $1776-82$

Avraham, F., Aviv, S., Ya'akobi, P., Faran, H., Fisher, Z., Goldman, Y., Neeman, G., Carmeli, E. The efficacy of treatment of different intervention programs for patellofemoral pain syndromeA single blinded randomized clinical trial. The Scientific World Journal 2007; 7: 1256-1262

Baker, G. R. The contribution of case study research to knowledge of how to improve quality of care. BMJ Quality and Safety 2011; 20 Supplement 1: i30-35.

Baldon, R., Serrão, F.V., Silva, R.S., Piva, S.R. Effects of functional stabilization training on pain, function and lower extremity biomechanics in females with patellofemoral pain: A randomized clinical trial. Journal of Orthopaedic and Sports Physical Therapy 2014; 44(4): 240-A8

Barton, C.J., Lack, S., Malliaras, P., Morrissey, D. Gluteal muscle activity and patellofemoral pain: A systematic review. British Journal of Sports Medicine2013; 47(4): 207-214

Barton, C.J., Munteanu, S.E., Menz, H.B. The efficacy of foot orthoses in the treatment on individuals with patellofemoral pain syndrome: A systematic review. Sports Medicine 2010; 40: 377-395

Berbano, E. P., Baxi, N. Impact of patient selection in various study designs: identifying potential bias in clinical results. Southern Medical Journal 2012; 105(3): 149-155.

Besier, T. F., Gold, G. E., Delp, S. L., Fredericson, M., \& Beaupre, G. S. The influence of femoral internal and external rotation on cartilage stresses within the patellofemoral joint. Journal of Orthopaedic Research 2008; 26(12): 1627-1635.

Blond, L., \& Hansen, L. Patellofemoral pain syndrome in athletes: a 5.7-year retrospective follow-up study of 250 athletes. Acta Orthopaedica Belgica 1998; 64(4): 393-400.

Bolgla, L.A., Earl-Boehm, J., Emery, C., Hamstra-Wright, K., Ferber, R. Comparison of hip and knee strength in males with and without patellofemoral pain. Physical Therapy in Sport 2015; 16(3): 215-221

Boling, M., Bolgla, L. A., Mattacola, C. G., Uhl, T. L., Hosey, R. G. Outcomes of a weight-bearing rehabilitation program for patients diagnosed with patellofemoral pain syndrome. Archives of Physical Medicine and Rehabilitation 2006; 87(11): 1428-1435.

Boling, M., Padua, D., Marshall, S., Guskiewicz, K., Pyne, S., Beutler, A. Gender differences in the incidence and prevalence of patellofemoral pain syndrome. Scandinavian Journal of Medicine and Science in Sports 2010; 20(5): 725-730. 
Boling, M., Padua, D. Relationship between hip strength and trunk, hip and knee kinematics during a jump landing task in individuals with patellofemoral pain. International Journal of Sports Physical Therapy 2013; 8(5): 661-669

Brent, J.L., Myer, G.D., Ford, K.R., Paterno, M.V., Hewett, T.E. The effect of age and gender on isokinetic hip abduction torques. Journal of Sport Rehabilitation 2013; 22: 41-46

Callaghan, M. J., Selfe, J. Has the incidence or prevalence of patellofemoral pain in the general population in the United Kingdom been properly evaluated? Physical Therapy in Sport 2007; 8: 37-43.

Charnock, B. L., Lewis, C. L., Garrett, W. E., Jr., Queen, R. M. Adductor longus mechanics during the maximal effort soccer kick. Sports Biomechanics 2009: 8(3): 223-234.

Cichanowski, H. R., Schmitt, J. S., Johnson, R. J., Niemuth, P. E. Hip strength in collegiate female athletes with patellofemoral pain. Medicine and Science in Sports and Exercise2007; 39(8): 1227-1232.

Collins, N.J., Bisset, L.M., Crossley, K.M., Vicenzino, B. (2012). Efficacy of nonsurgical interventions for anterior knee pain. Sports medicine 2012; 42(1) 31-49

Coppack, R. J., Etherington, J., Wills, A. K. The Effects of Exercise for the Prevention of Overuse Anterior Knee Pain: A Randomized Controlled Trial. American Journal of Sports Medicine 2011; 39(5): 940-948.

Crow, J., Pizzari, T., Buttifant, D. Muscle onset can be improved by therapeutic exercise: a systematic review. Physical Therapy in Sport 2011; 12(4): 199-209.

Crossley, K.M., Bennell, K.L., Cowan, S.M., Green, S. Analysis of outcome measures for persons with patellofemoral pain: Which are reliable and valid? Archives of physical medicine and rehabilitation 2004; (85): 815-822

Davis, I. S., Powers, C. M. Patellofemoral pain syndrome: proximal, distal, and local factors, an international retreat, April 30-May 2, 2009, Fells Point, Baltimore, MD. Journal of Orthopaedics and Sports Physical Therapy 2010; 40(3): A1-16.

Dayan, E., Cohen, L.G. Neuroplasticity subserving motor skill learning. Neuron 2011; 72(3): 443-454

Doherty, S. Evidence based medicine: arguments for and against. Emergency medicine Australasia 2005; 17(4) 307-313

Dolak, K. L., Silkman, C., Medina McKeon, J., Hosey, R. G., Lattermann, C., Uhl, T. L. Hip strengthening prior to functional exercises reduces pain sooner than quadriceps strengthening in females with patellofemoral pain syndrome: a randomized clinical trial. Journal of Orthopaedic and Sports Physical Therapy 2011; 41(8): 560-570.

Earl, J. E., Hoch, A. Z. A proximal strengthening program improves pain, function, and biomechanics in women with patellofemoral pain syndrome. American Journal of Sports Medicine 2011; 39(1): 154-163. 
Ferber, R., Kendall, K., Farr, L. Changes in Knee Biomechanics After a Hip-Abductor Strengthening Protocol for Runners With Patellofemoral Pain Syndrome. Journal of Athletic Training 2011; 46(2): 142-149.

Ferber, R., Bolgla, L., Earl-Boehm, J.E., Emery, C., Hamstra-Wright, K. Strengthening of the Hip and Core Versus Knee Muscles for the Treatment of Patellofemoral Pain: A Multicenter, Randomized Controlled Trial Journal of Athletic Training 2014; 49(3): doi: 10.4085/10626050-49.3.70

Fukuda, T. Y., Rossetto, F. M., MagalhÃes, E., Bryk, F. F., Lucareli, P. R. G., Carvalho, N. Short-term effects of hip abductors and lateral rotators strengthening in females with patellofemoral pain syndrome: a randomized controlled clinical trial. Journal of Orthopaedic and Sports Physical Therapy 2010; 40(11): 736-742.

Fukuda, T.Y., Melo, W.P., Zaffalon, B.M., Rossetto, F. M., MagalhÃes, E.,Bryk, F.F., Martin, R.L. Hip posterolateral musculature strengthening in sedentary women with patellofemoral pain syndrome: A randomized clinical trail with 1 year follow up. Journal of Orthopaedic \& Sports Physical Therapy 2012; 42(10): 823-830.

Gottschall, J.S., Okita,N., Sheehan, R.C. Muscle activity of the tensor fascia lata and adductor longus for ramp and stair walking. Journal of Electromyography and Kinesiology 2012; 22(1): 67-73.

Groah, S.L., Libin, A., Lauderdale, M., Kroll, T., DeJong, G., Hsieh, J. Beyond the evidence-based practice paradigm to achieve best practice in rehabilitation medicine: a clinical review. Physical Medicine and Rehabilitation 2009; 1 (10):941-950

Grossman, J., Mackenzie, F.J. The randomised controlled trial. Gold standard orsimply standard. Perspectives in medicine 2005;48(4): 516-534

Heintjes E. M, B. M., Bierma-Zeinstra Sita MA, Bernsen Roos MD, Verhaar Jan AN, Koes Bart W. Exercise therapy for patellofemoral pain syndrome. Cochrane Database of Systematic Reviews 2003.

Higgins, J. P., Altman, D. G., Gotzsche, P. C., Juni, P., Moher, D., Oxman, A. D., Cochrane Statistical Methods, G. The Cochrane Collaboration's tool for assessing risk of bias in randomised trials. BMJ 2011; 343: d5928.

Hodges, P. Pain and motor control: from the laboratory to rehabilitation. Journal of Electromyography and kinesiology 2011; 21: 220-228.

Huberti, H. H., Hayes, W. C. (1984). Patellofemoral contact pressures. The influence of q-angle and tendofemoral contact. Journal of Bone and Joint Surgery Am 1984; 66(5): 715-724.

Ismail, M.M., Gamaleldein, M.H., Hassa, K.A. Closed kinetic chain exercises with or without additional hip strengthening exercises in management of patellofemoral pain syndrome: a randomized controlled trial. European Journal of Physical Rehabilitation Medicine 2013; 49(5): 687-698.

Khayambashi, K., Mohammadkhani, Z., Ghaznavi, K., Lyle, M. A., Powers, C. M. The effects of isolated hip abductor and external rotator muscle strengthening on pain, health status, and 
hip strength in females with patellofemoral pain: a randomized controlled trial. Journal of Orthopaedic and Sports Physical Therapy 2012; 42(1): 22-29.

Khayambashi, K., Fallah, A., Movahedi, A., Bagwell, J., Powers, C. Posterolateral hip muscle strengthening versus quadriceps strengthening for patellofemoral pain: A comparitive control trial. Archives of Physical Medicine and Rehabilitation 2014; 95(5): 900-907

Koes, B. W., Hoving, J. L. The value of the randomised clinical trial in the field of physiotherapy. Manual Therapy 1998; 3(4): 179-186.

Kraemer, W.J., Rataness, N.A. Fundamentals of resistance training: progression and exercise prescription. Medicine and Science in sports and exercise 2004; 674-688.

Lee, T. Q., Morris, G., Csintalan, R. P. The influence of tibial and femoral rotation on patellofemoral contact area and pressure. Journal of Orthopaedic and Sports Physical Therapy 2003; 33(11): 686-693.

Leighton, R. D. A functional model to describe the action of the adductor muscles at the hip in the transverse plane. Physiotherapy Theory and Practice 2006; 22(5); 251-262.

Li, G., DeFrate, L. E., Zayontz, S., Park, S. E., Gill, T. J. The effect of tibiofemoral joint kinematics on patellofemoral contact pressures under simulated muscle loads. Journal of Orthopaedic Research 2004; 22(4): 801-806.

Lowry, C.D., Cleland, J., Dyke, Management of Patients with Patelllofemoral Pain Syndrome using a multi-modal approach: A case series. Journal of Orthopaedic and Sports Physical Therapy 2008; 38(11):691-702

Luft A.R., Buitrago,M.M. Stages of motor skill learning. Molecular Neurobiology 2005; 32(3): 205216.

MagalhÃes E., Fukuda, T. Y., Sacramento, S. N., Forgas, A., Cohen, M., \& Abdalla, R. J. A comparison of hip strength between sedentary females with and without patellofemoral pain syndrome. Journal of Orthopaedic and Sports Physical Therapy 2010; 40(10): 641-647.

Mascal, C. L., Landel, R., Powers, C. Management of patellofemoral pain targeting hip, pelvis, and trunk muscle function: 2 case reports. Journal of Orthopaedic and Sports Physical Therapy 2003; 33(11): 647-660.

Milanese, $\mathrm{S}$. The use of RCT's in manual therapy-are we trying to fit a round peg into a square hole? Manual Therapy 2011; 16(4): 403-405.

Nakagawa, T., Muniz, T., Baldon, R., Maciel, C., Reiff, R., Serrão, F. The effect of additional strengthening of hip abductor and lateral rotator muscles in patellofemoral pain syndrome: a randomized controlled pilot study. Clinical Rehabilitation 2008; 22(12): 1051-1060.

Nakagawa, T., Moriya, E. T., Maciel, C. D., Serrao, F. V. Trunk, pelvis, hip, and knee kinematics, hip strength, and gluteal muscle activation during a single-leg squat in males and females with and without patellofemoral pain syndrome. Journal of Orthopaedic and Sports Physical Therapy 2012; 42(6): 491-501. 
Neumann, D. Kinesiology of the hip: A focus on muscular actions. Journal of Orthopaedic and Sports Physical Therapy 2010; 40(2):82-94.

Nijs, J., Van Geel, C., Van der auwera, C., Van de Velde, B. Diagnostic value of five clinical tests in patellofemoral pain syndrome. Manual Therapy 2006; 11(1): 69-77.

Noehren, B., Schoz, J., \& Davis, I. The effect of real-time gait retraining on hip kinematics, pain and function in subjects with patellofemoral pain syndrome. British Journal of Sports Medicine 2011; 45(9): 691.

OCEBM Levels of Evidence Working Group. The Oxford 2011 Levels of Evidence.(2011) http://www.cebm.net/ocebm-levels-of-evidence/

Piva, S.R., Gil, A.B., Moore, C.G., Fitzgerald, G.K. Responsiveness of the activities of daily living scale of the knee outcome survey and numerical pain rating scale in patients with patellofemoral pain. Journal of Rehabilitation Medicine 2009; 41: 129-135

Powers, C. Patellofemoral pain: Proximal, Distal and Local factors 2nd International Research Retreat. Journal of Orthopaedic and Sports Physical Therapy 2012; 42(6): A1-A20.

Ramskov, D., Pedersen, M.B., Kastrup, K., Lonbro, S., Jacobsen, J.S., Thorborg, K., Nielsen, R.O., Rasmussen. S. Normative values of eccentric hip abduction strength in novice runners: An equation adjusting for age and gender. International Journal of Sports Physical Therapy 2014; 9(1): 68-75.

Rathleff, M.S., Rathleff, C.R., Crossley, K.M., Barton, C.J. Is hip strength a risk factor for patellofemoral pain? A systematic review and meta-analysis. British Journal of Sports Medicine 2014; 48(14): 1088-

Razeghi, M., Etamadi, Y., Taghizadeh, Sh., Ghaem, H. Could hip and knee strengthening alter the pain intensity in patellofemoral pain syndrome. Iranian Red Crescent Medical Journal 2010; 12(2):104-110

Remple, M.S., Bruneau, R.M., VandenBerg, P.M., Goertzen, C., Klein, J. Sensitivity of cortical representations to motor experience: evidence that skill learning but not strength training induces cortical reorganisation. Behavioural Brain Research 2001; 123: 133-141.

Reiman, M. P., Bolgla, L. A., Loudon, J. K. A literature review of studies evaluating gluteus maximus and gluteus medius activation during rehabilitation exercises. Physiotherapy Theory and Practice 2012; 28(4):257-268

Salsich, G. B., Perman, W. H. (2007). Patellofemoral joint contact area is influenced by tibiofemoral rotation alignment in individuals who have patellofemoral pain. Journal of Orthopaedic and Sports Physical Therapy 2007; 37(9): 521-528.

Sharp, K. The case for case studies in nursing research: the problem of generalization. Journal of Advanced Nursing 1998; 27(4): 785-789.

Song, C. Y., Lin, Y. F., Wei, T. C., Lin, D. H., Yen, T. Y., Jan, M. H. Surplus value of hip adduction in legpress exercise in patients with patellofemoral pain syndrome: a randomized controlled trial. Physical Therapy 2009; 89(5): 409-418. 
Souza, R. B., Draper, C. E., Fredericson, M., Powers, C. M. Femur rotation and patellofemoral joint kinematics: a weight-bearing magnetic resonance imaging analysis. Journal of Orthopaedic and Sports Physical Therapy 2010; 40(5): 277-285.

Souza, R. B., Powers, C. M. Differences in hip kinematics, muscle strength, and muscle activation between subjects with and without patellofemoral pain. Journal of Orthopaedic and Sports Physical Therapy 2009; 39(1): 12-19.

Taunton, J. E., Ryan, M. B., Clement, D. B., McKenzie, D. C., Lloyd-Smith, D. R., Zumbo, B. D. A retrospective case-control analysis of 2002 running injuries. British Journal of Sports Medicine 2002; 36(2): 95-101.

Tyler, T. F., Nicholas, S. J., Mullaney, M. J., McHugh, M. P. The role of hip muscle function in the treatment of patellofemoral pain syndrome. American Journal of Sports Medicine 2006; 34(4): 630-636.

van der Heijden, R.A., Lankhorst, N.E., van Linschoten, R., Bierma-Zienstra, S.M.A, van Middelkoop, M. Exercise for treating patellofemoral pain. The Cochrane Collaboration 2015

Willson, J. D., Binder-Macleod, S., Davis, I. S. Lower extremity jumping mechanics of female athletes with and without patellofemoral pain before and after exertion. American Journal of Sports Medicine 2008; 36(8): 1587-1596.

Willy, R.W., Manal, K.T., Witvrouw, E.E., Davis, I.S. Are mechanics different between male and female runners with patellofemoral pain? Medicine and Science in Sports and Exercise 2012a; 44(11): 2165-2171.

Willy, R.W., Scholz, J.P., Davis, I.S. Mirror gait retraining for the treatment of patellofemoral pain in female runners. Clinical Biomechanics 2012b; 27(10): 1045-1051.

Willy, R.W., Davis, I.S. Varied response to mirror gait retraining of gluteus medius control, hip kinematics, pain and function in two female runners with patellofemoral pain. Journal of Orthopaedic and Sports Physical Therapy 2013; 43(12): 864-874.

Witvrouw, E., Lysens, R., Bellemans, J., Cambier, D., Vanderstraeten, G. Intrinsic risk factors for the development of anterior knee pain in an athletic population. A two-year prospective study. American Journal of Sports Medicine 2000; 28(4): 480-489. 
Appendix 1

Search results

\begin{tabular}{|c|c|c|c|c|c|}
\hline & Pubmed & AMED & Cinahl & Sportdiscus & Embase \\
\hline Arthralgia & 11952 & 152 & 1981 & 56 & 4153 \\
\hline $\begin{array}{l}\text { "Knee joint" or } \\
\text { knee or patella }\end{array}$ & 55163 & 9661 & 38088 & 35631 & 158499 \\
\hline \#1 AND \#2 & 1100 & 61 & 522 & 12 & 3658 \\
\hline $\begin{array}{l}\text { "anterior knee } \\
\text { pain" }\end{array}$ & 1116 & 128 & 395 & 538 & 1397 \\
\hline $\begin{array}{l}\text { Femoropatell* OR } \\
\text { femoro-patell* OR } \\
\text { retropatell* OR } \\
\text { "patellofemoral } \\
\text { pain syndrome" OR } \\
\text { "patellofemoral } \\
\text { pain" }\end{array}$ & 1794 & 402 & 1147 & 1084 & 1926 \\
\hline $\begin{array}{l}\text { "lateral } \\
\text { compression } \\
\text { syndrome" OR } \\
\text { "lateral facet } \\
\text { syndrome" OR } \\
\text { "lateral pressure } \\
\text { syndrome" OR } \\
\text { "facet syndrome" }\end{array}$ & 130 & 21 & 28 & 21 & 225 \\
\hline $\begin{array}{l}\text { Chondromalac* OR } \\
\text { chondropath* }\end{array}$ & 1205 & 40 & 149 & 393 & 5756 \\
\hline $\begin{array}{l}\# 3 \text { OR \#4 OR \#5 OR } \\
\# 6 \text { OR \#7 }\end{array}$ & 4721 & 40 & 149 & 3895 & 11981 \\
\hline $\begin{array}{l}\text { Glute* OR } \\
\text { proximal OR hip } \\
\text { OR trunk }\end{array}$ & 470444 & 8485 & 49990 & 25091 & 422058 \\
\hline $\begin{array}{l}\text { Exercise OR } \\
\text { rehabilitation OR } \\
\text { strength OR } \\
\text { endurance OR } \\
\text { "motor control" }\end{array}$ & 836171 & 83345 & 230029 & 271019 & 796429 \\
\hline \#9 AND \#10 & 37276 & 4144 & 9382 & 10280 & 33424 \\
\hline \#8 AND \#11 & 251 & 804 & 3 & 40 & 392 \\
\hline
\end{tabular}

